\title{
Hierarchical Mechanical Properties of Wood Cell Wall in Microscale and Nanoscale
}

\section{Dissertation}

zur Erlangung des mathematisch-naturwissenschaftlichen Doktorgrades

"Doctor rerum naturalium"

der Georg-August-Universität Göttingen

\author{
im Promotionsstudiengang Physik \\ der Georg-August University School of Science (GAUSS)
}

vorgelegt von

Salimeh Saleh

aus Shahreza, Iran

Göttingen, 2021 


\section{Betreuungsausschuss}

Prof. C. A. Volkert, Institut für Materialphysik, Universität Göttingen

Prof. H. Militz, Abteilung Holzbiologie und Holzprodukte, Universität Göttingen

Prof. P. Vana, Institut für Physikalische Chemie, Universität Göttingen

Prof. A. Polle, Forstbotanik und Baumphysiologie, Universität Göttingen

\section{Mitglieder der Prüfungskommission}

Referentin: Prof. C. A. Volkert, Institut für Materialphysik

Korreferent: Prof H. Militz, Abteilung Holzbiologie und Holzprodukte

\section{Weitere Mitglieder der Prüfungskommission}

Prof. A. Polle, Forstbotanik und Baumphysiologie

Prof. M. Seibt, IV. Physikalisches Institut

Prof. T. Weitz, I. Physikalisches Institut

Prof. G. Ohms, Fakultät Ingenieurwissenschaften und Gesundheit, Hochschule für angewandte Wissenschaft und Kunst

Tag der mündlichen Prüfung: 04.05.2021 


\section{Contents}

$\begin{array}{lll}1 & \text { Introduction } & 7\end{array}$

1.1 The macroscopic structure of wood . . . . . . . . . . . 8

1.1.1 Wood classification as Hardwood and Softwood . . . . . . . . 8

1.1 .2 Growth rings . . . . . . . . . . . . . . 10

1.1.3 Different sectioning planes in wood . . . . . . . . . . 11

1.2 The microscopic architecture of the wood cell wall . . . . . . . . . . . . 12

1.3 Polymer components in the wood cell wall . . . . . . . . . . . . 15

1.3.1 Cellulose . . . . . . . . . . . . . . . . . . 15

1.3 .2 Lignin . . . . . . . . . . . . . . . . . . . 18

1.3.3 Hemicellulose . . . . . . . . . . . . . . . . . . . . . . . 19

1.4 Thermal treatment . . . . . . . . . . . . . . . . . 20

1.4.1 Chemical changes . . . . . . . . . . . . . . . . 21

1.4.2 Hygroscopicity and moisture content . . . . . . . . . . . . . 23 
1.4 .3 Mass loss . . . . . . . . . . . . . . . . . . . . 24

1.5 Mechanical measurements of wood . . . . . . . . . . . . . . . . 24

1.6 Objectives of the study . . . . . . . . . . . . . . . . . . . . 28

1.6.1 Micromechanical measurements of the wood cell wall by Nanoinden-

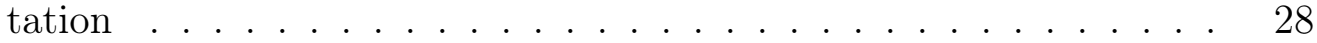

1.6.2 Nanoscale measurements of the wood cell wall by Atomic Force Microscopy . . . . . . . . . . . . . . . . . 28

2 Micromechanical measurements of the wood cell wall 31

2.1 Introduction . . . . . . . . . . . . . . . . . . . . 32

2.2 Materials and Methods . . . . . . . . . . . . . . . . . 35

2.2.1 Specimen preparation and characterization . . . . . . . . . . 35

2.2.2 Nanoindentation measurement methods . . . . . . . . . . . . . . . . 41

2.2.3 Positioning Nanoindents in the cell walls . . . . . . . . . . . . . . 48

2.3 Results . . . . . . . . . . . . . . . . . . . . 51

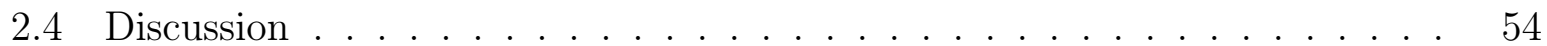

2.4.1 Composite model for the wood cell wall . . . . . . . . . . . . . 58

2.4.2 Origin of scattering in local mechanical properties . . . . . . . . . 63

2.4.3 Origin of the effect of thermal modification on micromechanical behavior ...................... . . . 65

2.4.4 Comparison of micromechanical behavior with macroscale properties $\quad 70$

2.5 Conclusion . . . . . . . . . . . . . . . . . . . . 72 
3 Ultrastructural arrangement of the wood cell wall in nanoscale

3.1 Introduction . . . . . . . . . . . . . . . . . . 76

3.2 Materials and Methods . . . . . . . . . . . . . . . . . . 78

3.2 .1 Sample preparation . . . . . . . . . . . . . . . 78

3.2 .2 Atomic Force Microscopy method . . . . . . . . . . . . . . . . . . . 79

3.3 Results and Discussion . . . . . . . . . . . . . . . . . . . . 88

3.3.1 AM-FM maps configuration for the wood cell wall . . . . . . . . 88

3.3.2 The effect of topography . . . . . . . . . . . . . . . . . . . . . 91

3.3.3 Comparing different regions of the wood cell wall . . . . . . . . . 95

3.4 Conclusion . . . . . . . . . . . . . . . . . . . 107

4 Summary 111

A Composite model for cell wall including interfaces 113

B Proposed model for nanostructural assembly of the wood cell wall $\quad 119$

$\begin{array}{ll}\mathrm{C} \text { Cell wall treatment } & 123\end{array}$

C.1 Delignification of the wood cell wall . . . . . . . . . . . . . 123

C.2 Cellulose degradation in the wood cell wall . . . . . . . . . . . . . 124 


\section{Introduction}

As one of the remarkable hybrid materials in nature, wood chooses the smartest way to bear mechanical loads by using the least amount of base materials and creates outstanding functional mechanical properties comparable with advanced composite materials.

Wood is a cellular biomaterial with a complex multi-component system. The excellent mechanical properties of wood are the consequence of its hierarchical structure at all length scales. Ranging from the meter-scale of the tree trunk, millimeter-scale of growth-rings, the microscopic scale of cellular structures such as tracheids, lumens, and cell walls, and the nanoscopic scale of the polymeric building blocks (e.g., cellulose, hemicellulose, and lignin) organization. This hierarchical architecture provides different properties for meeting the tree's diverse requirements and adapting to climate change.

Regarding the hierarchical structure of wood, the key challenge is developing mechanical characterization methods on the micro and nano scales to better understand the smallerscale mechanical properties and uncover whether small-scale architectures have unique properties that can affect the remarkable mechanical functionality of wood. Hence the micrometer and nanometer analysis were incorporated into the original goal of this research by implementation and development of nanoindentation and Atomic Force microscopy techniques. These two methods have been applied in this thesis to detect the ultrastructure and local mechanical properties of wood cell walls. The results allow the fundamental connections between the structure and mechanical response of wood to be revealed, including the 
important role of the cellulose-lignin interfaces in determining both stiffness and damping. This chapter summarises the background of wood anatomy and reviews literature about the wood cell wall's physical and mechanical properties.

\subsection{The macroscopic structure of wood}

\subsubsection{Wood classification as Hardwood and Softwood}

The two hardwood and softwood classes are one of the prominent categorizing of trees in the bulk scale. This classification is based on the differences in the physical properties and anatomy of wood.

Softwoods like pine, cedar, douglas fir, and redwood usually have needles (narrow leaves) and seeds generated in cones. These trees remain evergreen. About 90 percent of softwood is made up of longitudinal cells called tracheids arranged regularly and are responsible for water-conducting and mechanical support. The length of tracheids is about 100 times their diameter, reported as 2-4 $\mathrm{mm}$ in length and 0.025-0.045 $\mathrm{mm}$ in diameter $[1,2]$.

Softwoods also have rays that are extended in a radial direction across the growth rings (Figure 1.1(a) and Figure 1.3). They're narrower than the tracheids and are like thin straight lines. The wood rays aim to store and distribute the tree's photosynthesis products horizontally [3].

The hardwoods, such as eucalyptus, balsa, beech, oak, and walnut, lose their leaves annually, have broad leaves, and produce covered seeds within flowers. In hardwood, three different cells cause the main differences with softwood: vessels, fibers, and rays. A vessel cell is a series of relatively large diameter and thin-wall vessel elements connected end-toend. The vessels act like pipes that transport the water in the longitudinal direction, from the root to the tree's top. The vessels have a length of a few centimeters to more than 10 meters. The vessel elements are usually $25-300 \mu \mathrm{m}$ in diameter and $0.2-1 \mathrm{~mm}$ in length [1]. These vessels could be seen on hardwood's cross-sectional surface (Figure 1.1(b)). Therefore hardwoods are known as porous woods, and the presence of vessels (pores) on an 


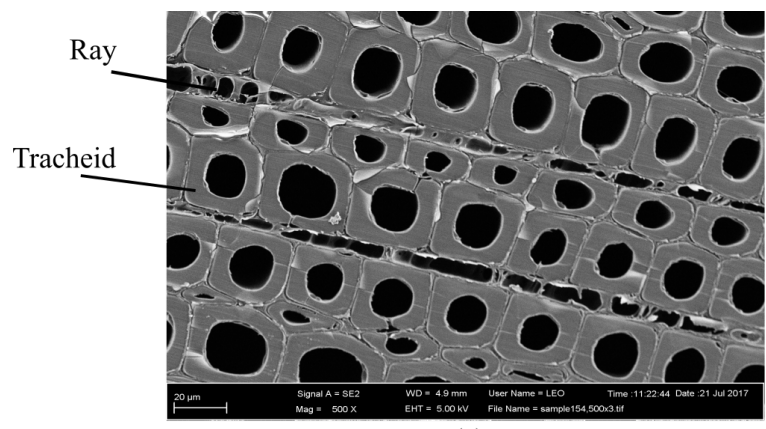

(a)

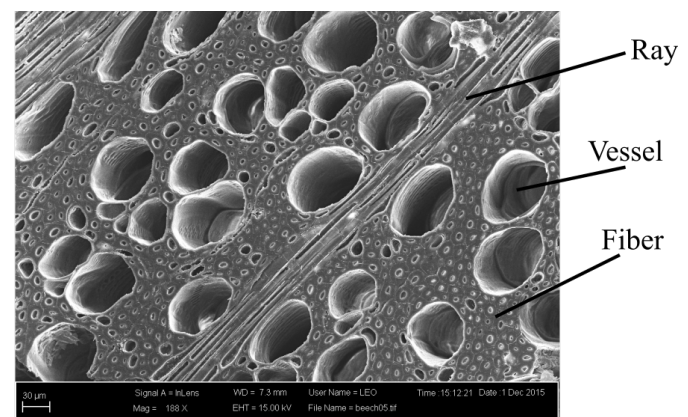

(b)

Figure 1.1: Scanning electron microscopy (SEM) images from the cross-section of (a) pine as softwood and (b) beech as hardwood. The different cell types, including rays, tracheids, fibers, and vessels, are shown in softwood and hardwood. The scale bars are $20 \mu \mathrm{m}$ in (a) and $30 \mu \mathrm{m}$ in (b).

unknown sample's cross-section specifies whether it is a hardwood or a softwood. The second element in hardwoods is the thick-walled longitudinal cells with different thicknesses called fibers responsible for mechanical support. The fibers usually have 10-30 $\mu \mathrm{m}$ diameter and 1-2 mm length [1]. The third essential element in hardwood is the rays. Although softwood and hardwood both have rays, however, the rays in hardwood are much more extensive and usually form $10-32 \%$ of the wood volume, but in softwood, they are narrow and are about $5-12 \%$ of the wood volume $[2,4]$.

The average density of hardwoods $\left(320-810 \mathrm{~kg} / \mathrm{m}^{3}\right.$, average: $\left.500 \mathrm{~kg} / \mathrm{m}^{3}\right)$ is slightly higher than softwoods $\left(290-600 \mathrm{~kg} / \mathrm{m}^{3}\right.$, average: $\left.410 \mathrm{~kg} / \mathrm{m}^{3}\right)$. Despite that, the terms softwood and hardwood could not help to differentiate these two categories' hardness because some hardwood species are softer than softwood species (and vice versa) [1].

In Figure 1.1, the cross-sectional views of pine as softwood and beech as hardwood are shown. The differences between fibers and tracheids in beech wood and pine wood are clear. Furthermore, the vessels are apparent in beech wood. 


\subsubsection{Growth rings}

Wood layers produced during the growing season have different colors and densities, which let the growth rings be recognized even without a microscope. Early in the growing season, the tree grows faster and produces large cells with light colors. Later in the growing season, the tree grows slower and produces tighter cells with dark colors. Therefore generally, two types of cells can be observed in an integrated growth ring: earlywood cells, with a thinner wall and wider lumen, act as a conductive and supporting element, and latewood cells, with a much thicker wall and narrow lumen, provide mechanical support.

The cell wall thickness is about $2-4 \mu \mathrm{m}$ in the earlywood and about $3-7 \mu \mathrm{m}$ in the latewood $[5,6]$. In Figure 1.2, the morphological differences between latewood and earlywood in the cross-section of pine wood are visible.

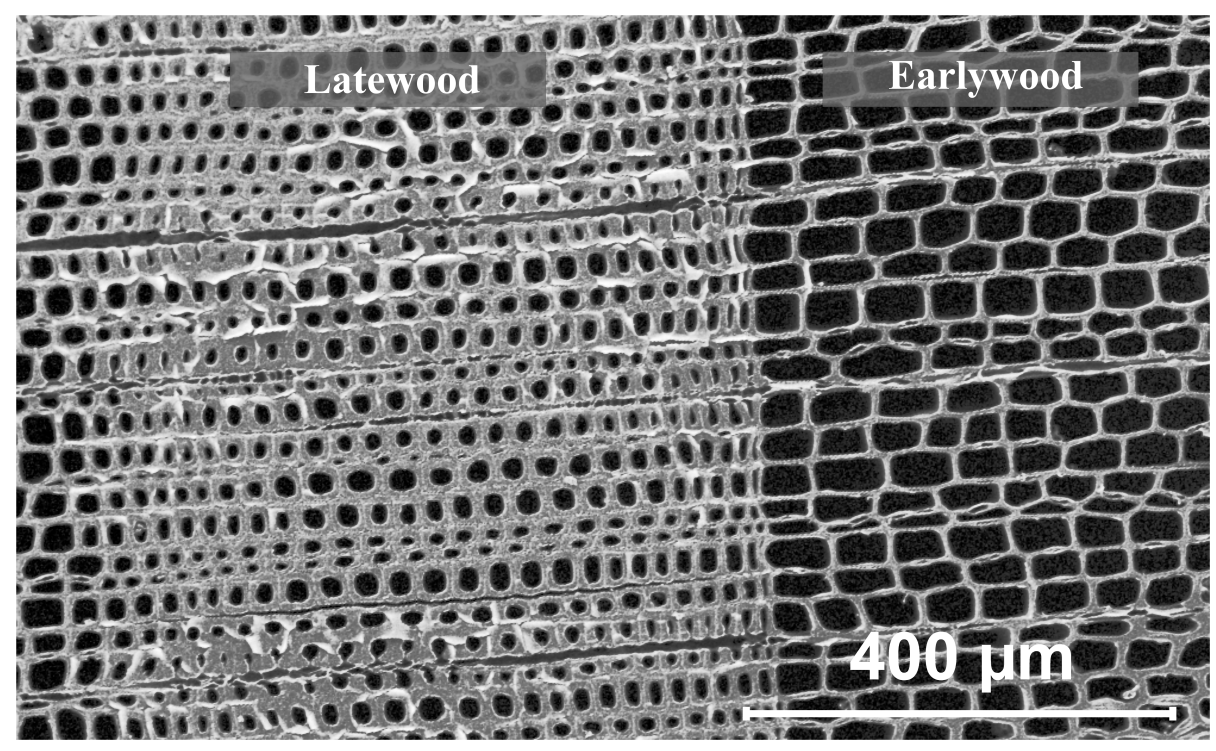

Figure 1.2: SEM image from the cross-section of pine wood, including latewood and earlywood.

In hardwoods, earlywood and latewood are more challenging to detect because the fiber diameter does not change during the growing season. The size and distribution of vessels vary in the growing season for some of the hardwood species. It has been observed that the vessels' size and distribution in earlywood are much larger than the latewood [2]. However, this kind of morphology change between latewood and earlywood is not identical for all 
hardwood species.

\subsubsection{Different sectioning planes in wood}

The wood structure is highly anisotropic and can be seen as an orthotropic. The three directions are: radial $(\mathrm{R})$ : normal to growth rings from pith to bark, tangential $(\mathrm{T})$ : circumferential to the growth rings, and longitudinal (L): along the tree axis and the grains (Figure 1.3). Each direction and section of a wood sample has its unique features and properties. The three different wood sections are included the transverse plane of section (the cross-section), the radial plane of section, and the tangential plane of section [7].

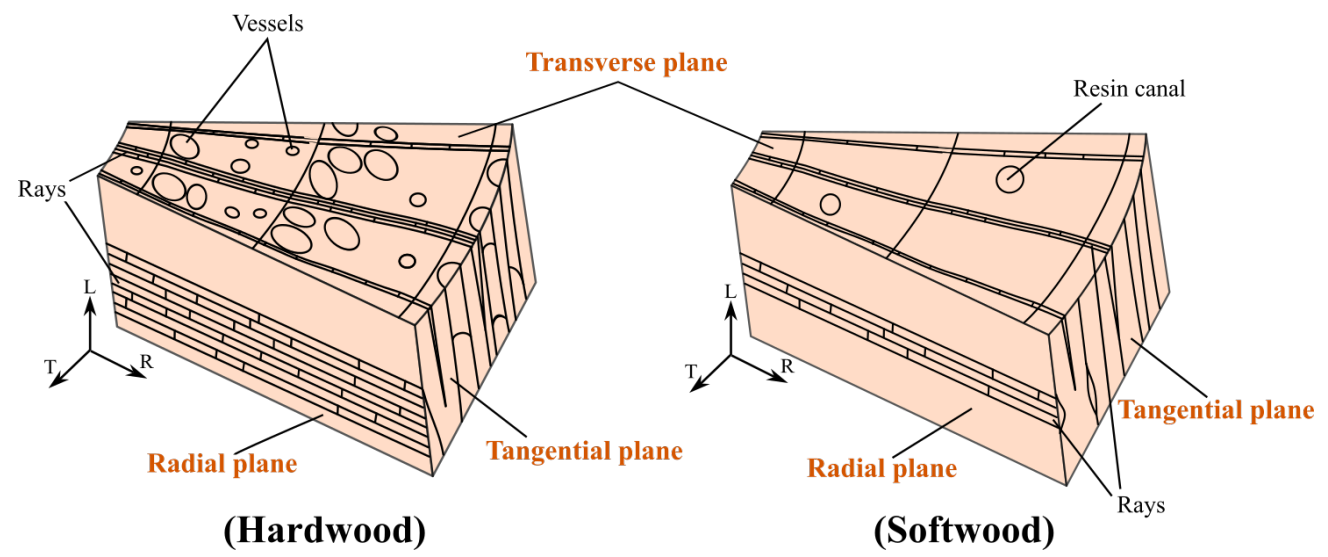

Figure 1.3: An overview of the wood coordinate system, including Longitudinal (L), Radial $(\mathrm{R})$, and tangential (T) directions. The different configurations of wood sectioning planes are shown for softwood and hardwood.

The transverse section is cut perpendicular to the stem's long axis and provides the features of growth rings, and represents the rays that are like narrow lines crossing the growth rings (Figure 1.3) [7]. When the tree is cut parallel to the stem's long axis (longitudinal cut), two different planes can be provided: tangential and radial. The tangential section is perpendicular to the rays and tangential to the annual rings. The profile of rays is visible in tangential sections (see Figure 1.3 and Figure 1.4).

The cutting direction in the radial section is parallel to the rays and perpendicular to the growth rings. The radial section provides the features of rays, which are well known to have a "brick-wall" appearance [1, 7]. 


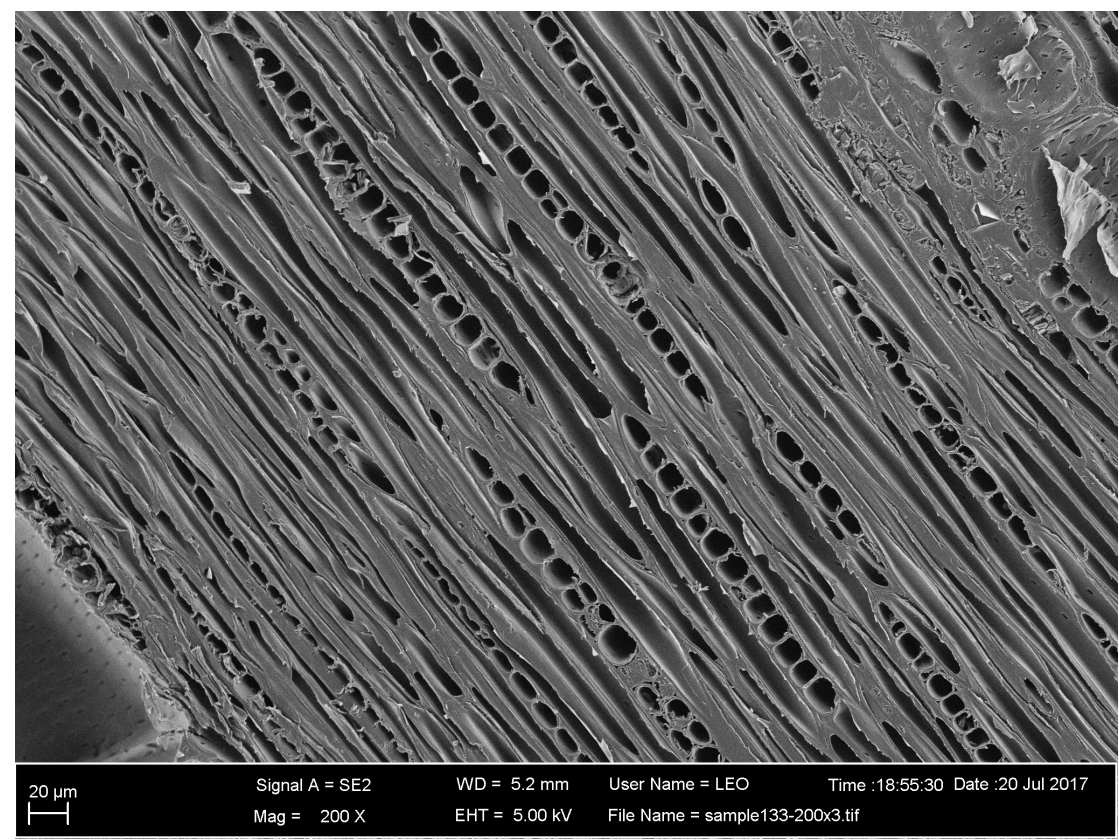

Figure 1.4: SEM micrograph from the tangential section. The profile of rays can be seen as rectangular shapes.

\subsection{The microscopic architecture of the wood cell wall}

At the microscopic level, the cell wall consists of three main layers: the middle lamella, the primary wall, and the secondary wall (Figure 1.5).

Each cell is attached to its neighbors by middle lamella. The primary wall is the next layer, which forms about $3 \%$ of the cell wall and is located inside the middle lamella. The primary wall is very thin and is challenging to distinguish from the middle lamella. Therefore, the term compound middle lamella (CML) defines the primary cell walls and the middle lamella of two adjacent cells. The compound middle lamella in wood is mostly lignified $[4,7,8]$.

As shown in Figure 1.5, the first layer in the secondary wall, adjacent to the compound middle lamella, is S1, a thin layer with a thickness of about $0.1-0.2 \mu \mathrm{m}$. The S2 layer is the central and thickest secondary wall, which makes up about 1-5 $\mu \mathrm{m}$ (70-80\%) of the cell wall thickness. It has the most significant contribution to the cell wall's properties. On the inner side of the S2 layer, there is the S3 layer adjacent to the cell lumen. It is a relatively 
thin wall layer with a thickness of $0.1-0.2 \mu \mathrm{m}[4,7,8]$.

In comparison to other secondary layers, the S3 layer has the lowest percentage of lignin. This phenomenon is explained by Wiedenhoeft et al. [7]. The lower fraction of lignin is related to the physiology of the living tree. For water transportation, a significant adhesion between the water molecules and the wood cells is required. Since lignin is a hydrophobic molecule, the S3 layer, as the cell wall's inner side, must have a lower lignin concentration to facilitate water transportation [7].

The cell wall structure is principally composed of cellulose, hemicelluloses, and lignin. To date, the arrangement and the interactions of these polymeric components at the nanometer scale have not been entirely resolved. The averaged volume fractions of these polymeric components in different wall layers are presented in Table 1.1, reported by Fengel et al. [9]. The highest fraction of lignin is in the compound middle lamella, and the highest fraction of cellulose is in the $\mathrm{S} 2+\mathrm{S} 3$ layers.

Table 1.1: Variations of different polymeric components in different wood cell wall layers [9].

\begin{tabular}{lccc}
\hline \hline Cell Wall layers & \multicolumn{3}{c}{ Chemical Constituents\% } \\
& Cellulose & Hemicellulose & Lignin \\
\hline Compound middle lamellae & 12.5 & 25.5 & 62.0 \\
Secondary wall, S1 & 34.5 & 35.5 & 30.0 \\
Secondary wall, S2+S3 & 55.7 & 14.3 & 30.0 \\
\hline \hline
\end{tabular}

It is reported that in the wood cell wall, cellulose chains are compacted into larger units called elementary fibrils that are aggregated to form threadlike fibers known as microfibrils $[9,10,11]$. The cellulose microfibrils have a helical orientation towards the wood cell wall. So from the mechanical point of view, the unique molecular structure and the particular orientation of the cellulose fiber nominate it as the most leading microstructural element in the wood cell wall.

The angle between the cellulose microfibrils' helical orientation and the longitudinal cell axis is called microfibril angle (MFA) [12]. The microfibril angle varies within a single wood cell wall. One of the most critical characteristics in different secondary layers of 
the wood cell wall is their microfibrils helical arrangements. It has been shown that in the primary layer, the cellulose microfibrils have no specific orientation and are randomly distributed. In the S1 and S3 layers, the microfibrils are oriented almost perpendicular to the longitudinal axis. While in the S2 layer, the microfibrils are mostly aligned to the longitudinal axis $[11,12,13,14]$. In the research of Donaldson et al. [15] microfibril orientation in the secondary cell wall of Radiata pine tracheids has been described. In the S1 layer, the microfibrils were often arranged in the S-helix $\left(>90^{\circ}\right)$, with an angle of $79^{\circ}$ to $117^{\circ}$. However, the irregular changing of the microfibrils' orientation from the $\mathrm{S}$ helix to the $\mathrm{Z}$ helix in the $\mathrm{S} 1$ layer was observed within individual tracheids. In the S2 layer, the microfibrils were arranged in a $\mathrm{Z}$ helix $\left(<90^{\circ}\right)$ orientation with an angle of $1^{\circ}$ to $59^{\circ}$. The microfibrils in the S3 layer also had a Z-helix orientation with an angle from $50^{\circ}$ to $113^{\circ}$. The microfibrils orientations in Figure 1.5 have been drawn according to Donaldson's explanation [14].

When wood is loaded in the longitudinal direction, the S2 layer plays the predominant role, and the cell wall acts as a spring with stiff behavior at low MFA of the S2 layer [16]. However, when the wood is loaded in the transverse direction, the S1 and S3 layers with the higher MFA have an essential mechanical contribution. Thus the exchange of MFA from S1(high angle) to S2 (low angle) and then S3 (high angle) represent an adaptional behavior of the wood cell wall to resisting compression, buckling, and bending forces in the tree [16]. Further, the cellulose fibril aggregates increase the tensile strength while the lignin enhances the compressive stability.

The uniformity and diversity of MFA in the radial wall and tangential wall of a single fiber are also different. Experiments show that the high variation of MFA is observable in the radial walls, which contain the bordered pits. However, the microfibril orientation in the tangential walls is less variable. This diversity is also higher in the radial wall of earlywood than latewood fibers [17].

The ultrastructural arrangement of the wood cell wall and the correlation of its polymeric components are still under debate. The main reason is the limitation of high-resolution optical microscopies and spectroscopies and the lack of non-destructive chemical and mechanical nanoscale measurements. Furthermore, no practical method has been developed to isolate cell wall components and keep their native 3D structures. 


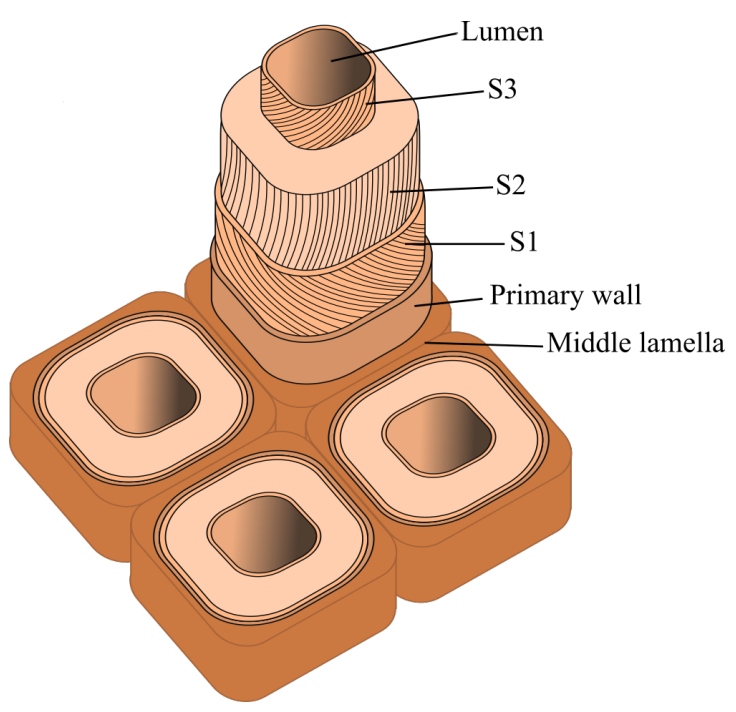

Figure 1.5: A schematic picture of the wood cell wall structure, including middle lamella, primary wall, and secondary layers. The microfibril arrangements in the secondary layers are indicated according to Donaldson's explanation [15].

So far, two different arguments from "Bailey [18]" and "Frey [19]" about the wood cell wall's ultrastructural arrangement have been demonstrated:

In 1938, Bailey reported that "the secondary walls are composed of a porous but firmly coherent matrix of anisotropic cellulose where lignin and other noncellulosic constituents may be deposited in the elongated, intercommunicating interstices of the cellulose [18]." Later, in 1968, Frey reported that "the wood cell wall resembles a reinforced concrete in which cellulose and lignin play the role of steel rods and concrete, respectively [19]." Frey's model is the most widely accepted cell wall ultrastructural model to date.

\subsection{Polymer components in the wood cell wall}

\subsubsection{Cellulose}

Cellulose is a linear polysaccharide consisting of almost $60 \%$ crystalline part linked to the $40 \%$ less crystalline or amorphous domains. As shown in Figure 1.6, the crystalline part of cellulose consists of linear chains of glucose joined by $\beta$-1, 4-glycosidic linkages [20]. 


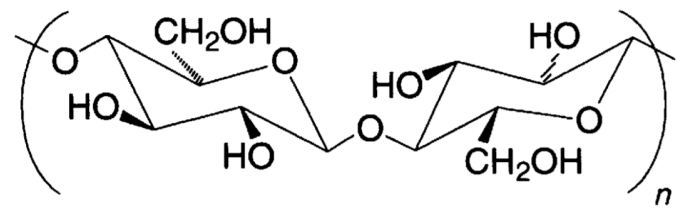

Figure 1.6: Cellulose chemical structure with the formula $\left(\mathrm{C}_{6} \mathrm{H}_{10} \mathrm{O}_{5}\right)_{n}$. Reprinted from T. Nishino [21], Copyright 2004, with permission from Elsevier.

There are several types of cellulose, including cellulose I, II, III, and IV. Cellulose I type is obtained from natural plants, known as the native cellulose, and is divided into two crystalline types, including cellulose $\mathrm{I} \alpha$ (triclinic) and cellulose $\mathrm{I} \beta$ (monoclinic) $[22,23]$. The relative amount of cellulose $\mathrm{I} \beta$ and cellulose $\mathrm{I} \alpha$ is different in different wood spices $[21,24]$.

Scientists have shown interest in the extraordinary mechanical properties of cellulose as a crystalline component. As shown in Figure 1.7, the elastic modulus of the perfect crystal of native cellulose was determined by Nishino et al., using the X-ray diffraction method. They monitored the crystal lattice spacing changes under constant stress. The stress-strain curve's initial slope was used to calculate the crystal modulus of cellulose, which was found to be $138 \mathrm{GPa}[21,25]$.

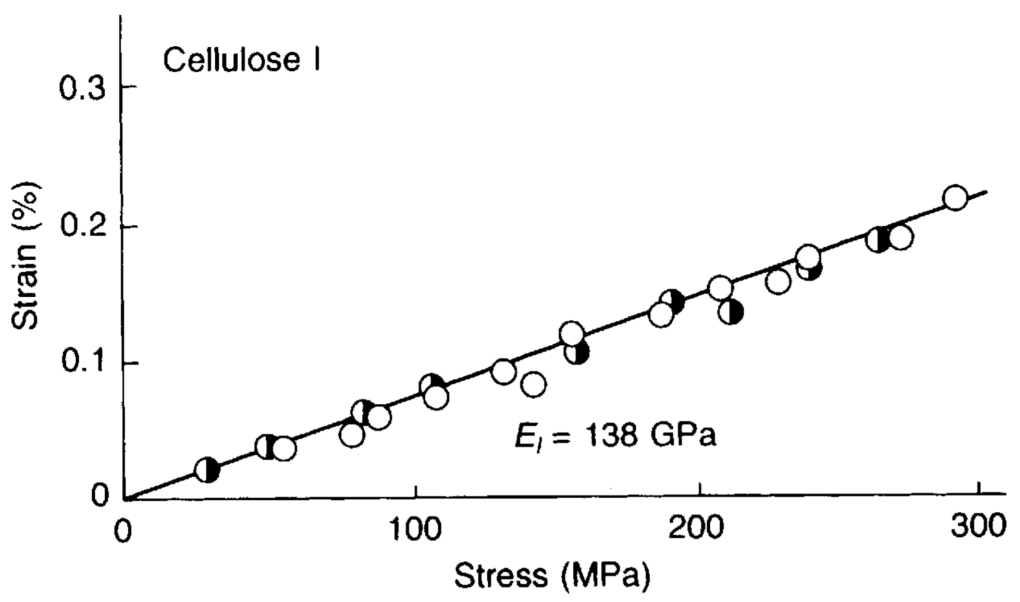

Figure 1.7: Stress-strain curve for the cellulose I. Open circles indicate the (004) plane, and the half-filled circles indicate the (008) plane in the crystalline structure. Reprinted from T. Nishino [21], Copyright 2004, with permission from Elsevier.

Tashiro and Kobayashi's observations [26] suggested that the most critical factor in deter- 
mining the elastic modulus of cellulose is the intramolecular hydrogen bonds. As shown in Figure 1.8, the skeletons of cellulose cannot elongate to its axial direction intrinsically by the lack of intramolecular hydrogen bonds. Consequently, it causes a decrease in the crystal modulus.
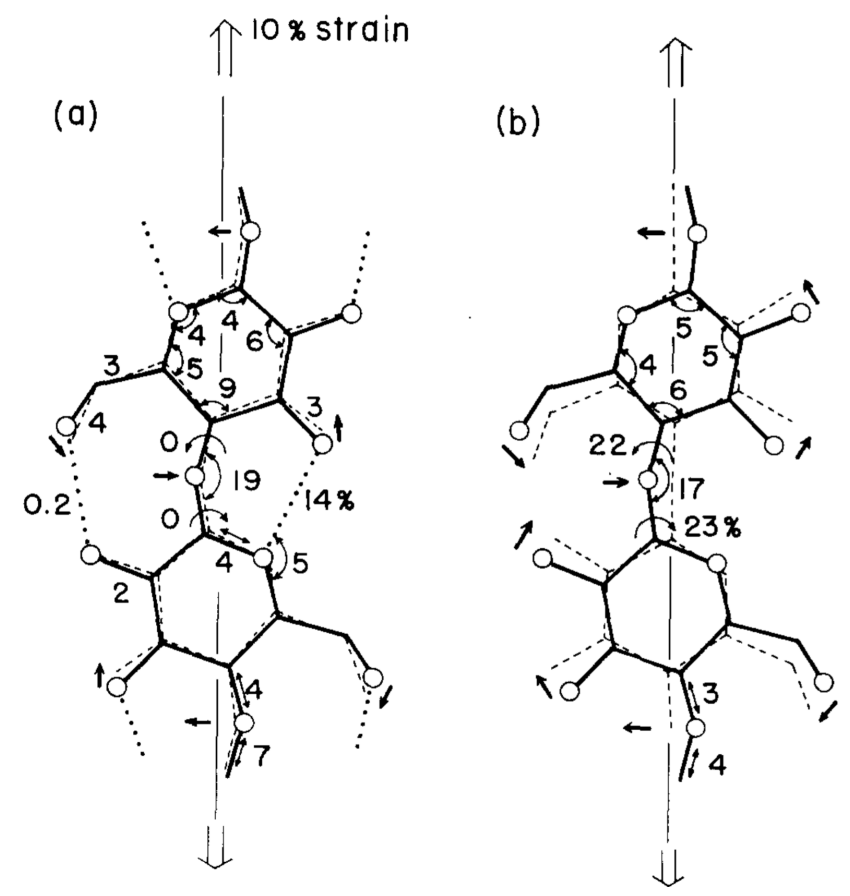

Figure 1.8: The calculated atomic displacements of cellulose chain under tensile force (a) with and (b) without the intramolecular hydrogen bonds. Reprinted from K. Tashiro et al. [26], Copyright 1991, with permission from Elsevier.

According to the Rowell and Winandy model [20], the polymeric chain and the crystallinity of cellulose are essential in the wood cell wall's mechanical behavior. The pyranose rings in cellulose structure are made of $\mathrm{C}-\mathrm{C}$ and $\mathrm{C}-\mathrm{O}$ covalent bonds. When a load is applied to the wood cell wall, C-C and C-O covalent bonds are getting shrunk or stretched or twisted within the pyranose ring structures at the molecular level (Figure 1.9). This could be a reason that cellulose is exceptionally resistant to tensile stresses [20]. The next factor which affects the elastic strength, explained by Rowell and Winandy, is the hydrogen bonds between and within individual polymer chains. Hydrogen bonds under stress can breaking, sliding and reforming and allow the stress to distribute within the structure [20]. 


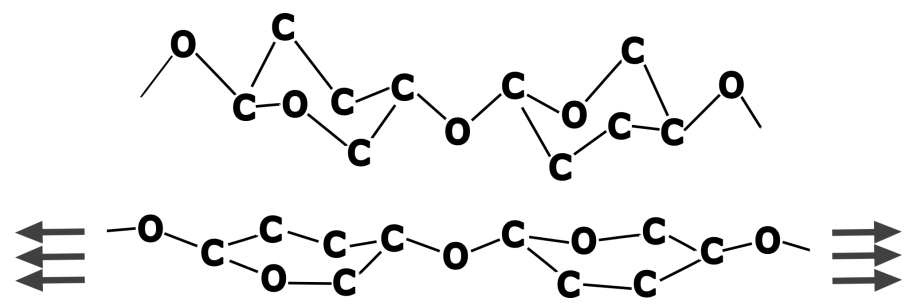

Figure 1.9: The schematic illustration of elongated polysaccharide molecules under tensile forces. Adopted with permission from J. E. Winandy et al. [20], Copyright 1984, American Chemical Society.

\subsubsection{Lignin}

Lignin is an amorphous, hydrophobic phenolic compound with a threedimensional organization that is considered to be a strengthened substance in the nano-structured assembly of the wood cell wall. As shown in Figure 1.10, lignin is made up of three different precursors, including p-coumaryl alcohol, coniferyl alcohol, and sinapyl alcohol. Biosynthesis of lignin in softwood and hardwood takes place with a wide variation of these precursors. In softwood, the predominant precursor of lignin is structure 2 (coniferyl alcohol), and in hardwood, the significant lignin precursors are the structure 2 and 3 . The lignin proportion of hardwoods is usually $18 \%-25 \%$, whereas, in softwoods, it varies between $25 \%$ and $35 \%[27,28]$.

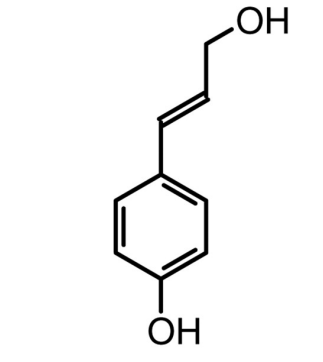

p-coumaryl alcohol<smiles>COc1cc(/C=C/CO)ccc1O</smiles>

coniferyl alcohol<smiles>COc1cc(/C=C/CO)cc(OC)c1O</smiles>

sinapyl alcohol

Figure 1.10: Chemical structures of lignin monolignols: p-coumaryl alcohol, coniferyl alcohol, and sinapyl alcohol. Reprinted from R. Hatfield et al. [29], copyright 2001, by permission of Oxford University Press.

Lignin's chemical structure differs from linear or branched carbohydrate chains. The hy- 
drophobic behavior of lignin excludes water from the hydrophilic carbohydrates. According to Roger Rowell's explanation, the stable strength and stiffness of wood, even in higher moisture content, are related to the lignin ability to limit water access to the carbohydrates and hydrogen-bonded structure [27].

Lignin is also introduced as nature's adhesive since the adjacent cells are attached by middle lamella, which has the highest lignin concentration. It should be mentioned that, although the middle lamella has the highest concentration of lignin, about $70 \%$ of lignin content is located in the secondary layer of the cell wall because of the higher volume of the secondary layer in comparison to the middle lamella.

According to Xue Kang et al. [30], lignin has the ability to self-aggregate and form hydrophobic nanodomains by extensive surface contact with hemicelluloses. However, there is no evidence that lignin is associated with cellulose [27].

\subsubsection{Hemicellulose}

Hemicellulose is the most common carbohydrate after cellulose in the plants. Hemicelluloses are a collection of polysaccharide polymers with a lower degree of polymerization (average DP of 100-200) than cellulose (average DP of 500-15000) [27]. The polymer chains in cellulose and hemicellulose are also different. Cellulose is a linear polymer without the attached side group composed of glucose, but the hemicelluloses are Y-branched and have attached side groups of different sugar monomers (Figure 1.11). Hemicellulose is the least thermally stable component in the cell wall and is closely associated with cellulose [27].

The overall structures of major wood hemicelluloses have not been entirely determined. Only the ratios of sugar monomer components in hemicellulose for different wood spices have been studied. It was proposed that the proportion of sugar monomer components is different in hardwood and softwood. Figure 1.12 shows the differences in hardwood and softwood for the distribution of lignin, cellulose, and various hemicelluloses in the cell wall layers. The predominant hemicellulose in hardwood is glucuronoxylan, while in softwood, glucomannan plays an essential role as hemicellulose. Furthermore, as shown in Figure 1.12, in the compound middle lamella, hemicellulose is the dominant carbohydrate, 

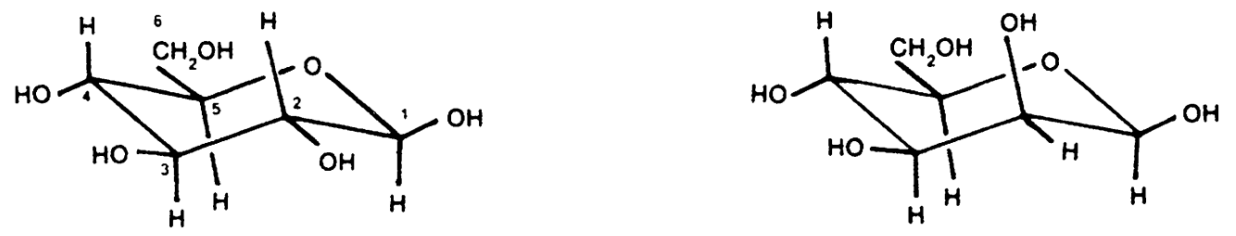

$$
\begin{aligned}
& \text { B-D.Glucose } \\
& \beta \text {-D.Glucopyranose } \\
& \beta \text {-D.Glup }
\end{aligned}
$$

$$
\begin{aligned}
& \text { B-D.Mannose } \\
& \text { B-D-Mannopyranose } \\
& \beta \text {-D.Manp }
\end{aligned}
$$
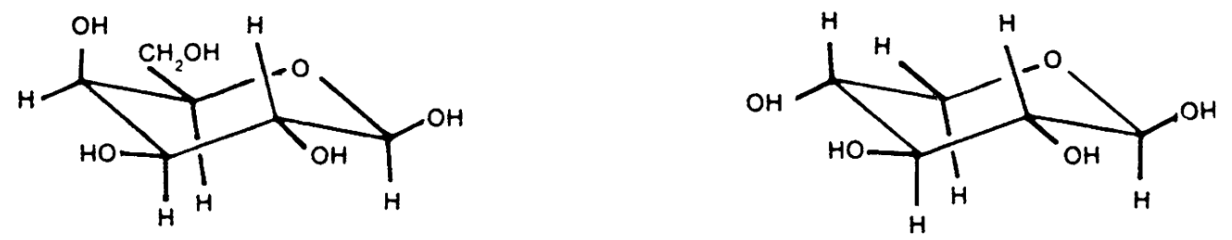

$$
\begin{aligned}
& \beta \text {-D.Galactose } \\
& \beta \text {-D.Galactopyranose } \\
& \beta \text {-D.Galp }
\end{aligned}
$$

\section{$\beta \cdot D-x y l o s e$}

B-D-Xylopyranose

$\beta-D-X y \mid p$

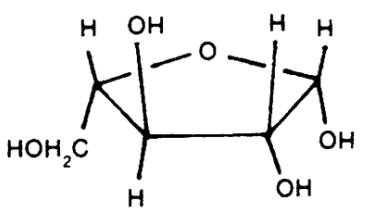

$\alpha \cdot L \cdot A r a b i n o s e$

$\alpha \cdot L \cdot$ Arabinofuranose

$\alpha \cdot L \cdot A r a f$

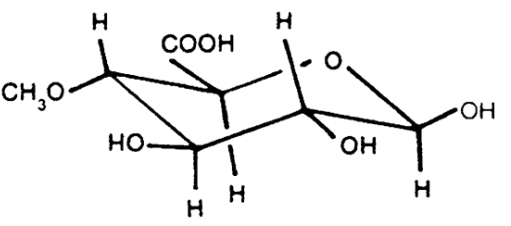

4-0. Methylgucuronic acid

4-O.Methylglucopyranosyluronic acid 4-O-Me- $\alpha$-D.GIUpA

Figure 1.11: Different monomers of hemicellulose in wood [27].

while in the secondary layers, cellulose is the essential carbohydrate [31].

\subsection{Thermal treatment}

In general, thermal treatment on wood aims to decrease hygroscopicity and improve dimensional stability and durability [32]. There are several different methods of thermal modification. Steam treatment is a hydrothermal method that causes a more effective heat 

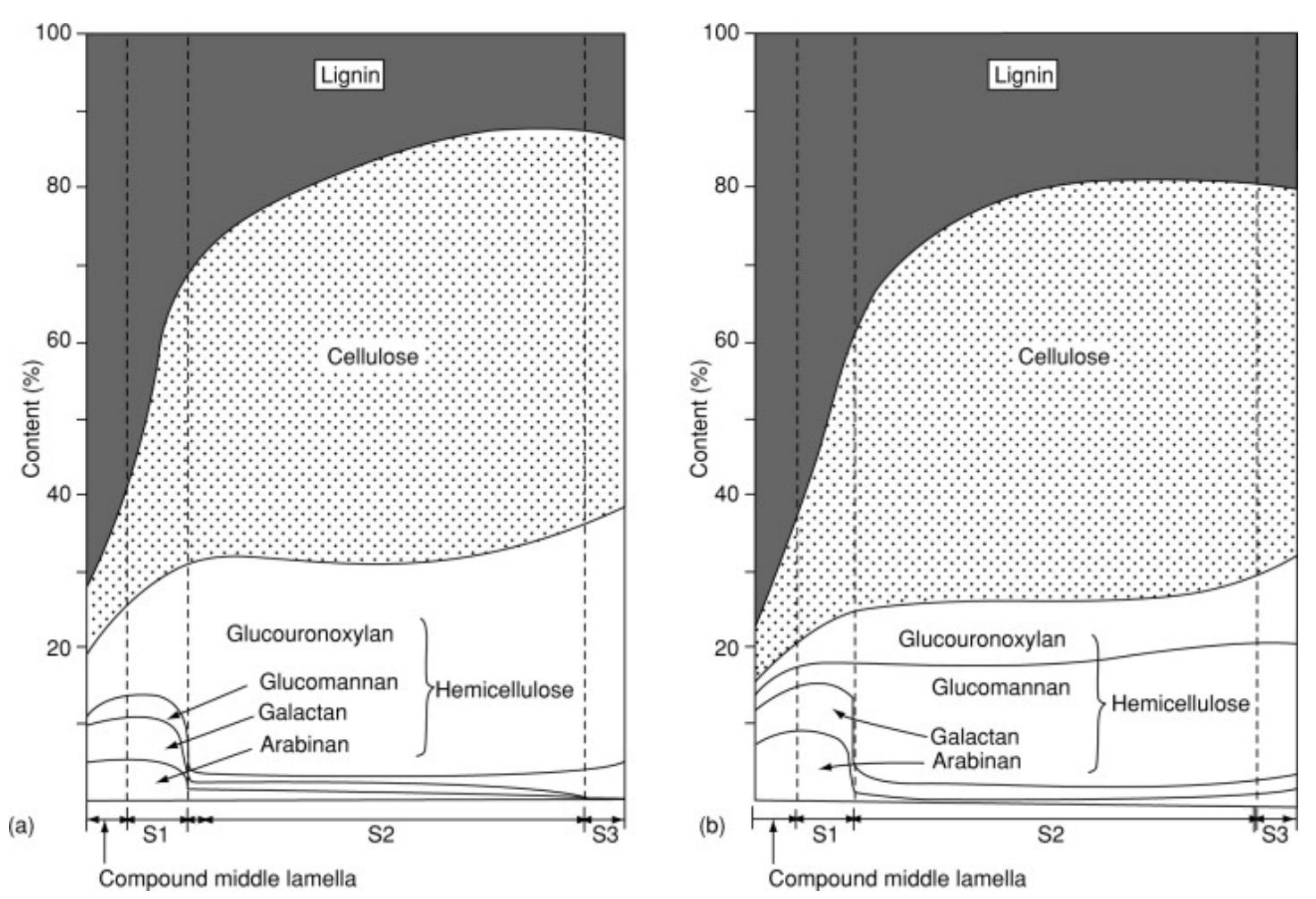

Figure 1.12: Distribution of lignin, cellulose, and hemicellulose in (a) hardwood and (b) softwood. Reprinted from M.T. Holtzapple [31], copyright 2003, with permission from Elsevier.

transfer, the limitation of oxygen in the process, and lower shrinkage stresses in the wood structure than conventional heat treatment methods. The combination of steam (humidity and pressure) and temperature (the maximum temperature of more than $120^{\circ} \mathrm{C}$ ) in the hydrothermal method results in different chemical and mechanical changes in the wood cell wall's structure [33], which will be described in the next section.

\subsubsection{Chemical changes}

In the hydrothermal method as a moist treatment, the hemicelluloses are the first structural compounds that are affected. Depolymerizing the hemicelluloses occurs at the first stage with the effect of hydronium ions generated by water as a catalyst [34, 35]. This depolymerizing involves cleavage of the hemicellulose constituents. Deacetylation of hemicelluloses by hydrolytic cleavage of the acetyl side chains produces acetic acid [36]. 
In the next reaction stages, the hydronium ions produced from acetic acid also act as catalysts, which causes the carbohydrates cleavage and, consequently, reduces the carbohydrates' degree of polymerization. The contribution of acetic acid as a catalyst is even more predominant than water autoionization, which increases the polysaccharides' decomposition. The acid catalyzation forms furfural, formaldehyde, and other aldehydes (a loss in hydrogen bonds) [32].

For cellulose fibers, as a crystallite part of the cell wall, thermal modification has the potential to change the crystallinity. Guo et al. founded that the cellulose crystallinity of cell walls increased considerably in both earlywood and latewood after compression steam treatment [37]. Thermal modification under moist conditions had the same influence on cellulose in the research of Bhuiyan et al. [38] and Ito et al. [39]. The experiments of Bhuiyan et al. [38] suggested that moist condition during thermal modification has much more effect on the crystallization of cellulose in comparison to the oven-dried condition. The increase in the crystallinity of wood included the thermal decomposition and crystallization of the amorphous region, resulting in decreasing the accessibility of hydroxyl groups to water molecules [32, 40].

In addition to cellulose crystallinity, a significant increase in the cellulose crystallite dimensions from 3.1 to $3.4 \mathrm{~nm}$ reported by Andersson et al. However, there were no considerable changes in the microfibril angle distribution in their observation [41]. The increase in the cellulose crystallite dimensions was reported in the other research as well [37, 42].

Although the glass transition temperature ( $\mathrm{Tg}$ ) of cellulose, determined by Salmen et al. [43], is about $220^{\circ} \mathrm{C}$, the presence of moisture could decrease this temperature [44]. Guo et al. reported that the rearrangement of adjacent cellulose chains to the higher crystallinity could happen at a temperature of $140^{\circ} \mathrm{C}$ or higher [37]. Lignin, as one of the main building blocks in the wood cell wall, is the most stable component during thermal modification. The thermal treatment leads to a relative increase in the lignin percentage and modification of the lignin polymer network. The polycondensation reactions and increase of cross-linking within the lignin-carbohydrate-complex are the other effects of thermal treatment on wood's molecular structure. This molecular change consequently improves the dimensional stability and decreases wood hygroscopicity [32, 45]. 
Most of the natural wood extractives tend to be degraded and produce new compositions after thermal modification. Evaporation of water and ethanol extractives due to the polysaccharides' degradation is one reason for the mass loss in the wood after thermal treatment [46].

\subsubsection{Hygroscopicity and moisture content}

Hygroscopicity is the material's ability to attract and hold water molecules to equilibrate from the surrounding environment. For wood, it is usually measured by the equilibrium moisture content (EMC). The equilibrium moisture content (EMC) of wood is the steadystate level of wood, in a particular relative humidity and temperature, in which the material is neither gaining nor losing moisture content [20].

The decrease of EMC is one of the most important effects on wood after thermal modification. It was found that after thermal treatment, the equilibrium moisture content has a significant decrease of up to $49.3 \%$ [47]. The lower moisture content in the wood means that less water is absorbed in the wood cell wall. It is the consequence of less available hydroxyl groups in the wood cell wall after thermal modification [20].

The degradation of hemicellulose in the thermal modification process causes the decrease of $\mathrm{OH}$ groups in the wood cell wall, and therefore the water absorption is mostly eliminated. The higher crystallinity of cellulose and the condensation reactions of lignin, which increase the cross-linking in the polymeric structure, limit water accessibility to hydroxyl groups as well.

The role of water in wood is different in different levels of moisture content. In the level of oven-dry ( $\sim 0 \% \mathrm{MC})[48]$ to the fiber saturation point $(\sim 28-30 \% \mathrm{MC})$ [48], the bounded water molecules play an influential role because changing the water content affects the strength of wood. Above the fiber saturation point, the free water molecules fill the wood cell cavities, and in this stage, changing the amount of moisture content does not change the strength of wood very much. According to the Rowell explanation, increasing the moisture content in the wood cell wall from oven-dry point to fiber saturation point increases the bonded water molecules in the structure and consequently causes the reduction of hy- 
drogen bonding between polymeric chains in the structure, which decreases the strength of wood [20].

\subsubsection{Mass loss}

Depolyerisation and degradation of wood cell wall components and extractives, the formation of new degradation products, and vaporization at high temperatures cause a mass loss in wood. This term usually uses to explain the intensity and degradation effects of thermal modification on wood. According to the explanation of Obataya et al. [49], the most error-free measurement of mass loss is obtainable when the extractives are deducting from the dry mass before and after modification. So the mass loss is measured after the extraction of all water-soluble degradation products. The experiments have shown that thermal treatment causes a significant decrease of $10.5 \%$ in the wood density [47].

\subsection{Mechanical measurements of wood}

The remarkable variety of mechanical properties in different wood species is derived from the polymeric components' arrangements in wood cell walls and cellular structure changes [4]. Furthermore, parameters such as density, fibrous orientation, and moisture content are known to affect the mechanical efficiency of wood on a bulk scale [50]. On a smaller scale, ultrastructural studies have shown that cellulose microfibrils' orientation influences wood properties $[51,52,53]$.

So far, single fiber micro tensile tests and nanoindentation methods have been the most widely used methods for determining the mechanical properties of wood cell walls experimentally.

The tensile test was one of the first measurements to understand wood's mechanical properties, and many different research techniques have been established till now [54, 55, 56, $57,58]$. Regarding these experiments, it was proposed that the shape of the stress-strain curve is highly structural-dependent. 
Reiterer et al. performed experimental tensile tests on the thin $(200 \mu \mathrm{m})$ sections of wood samples. They revealed that the variations of MFA offer the right balance of stiffness (small angles) and extensibility (large angles) for the wood cell walls [53].

Burgert et al. [59] carried out micro-tensile tests on mechanically and chemically isolated spruce fibers. They reported a mean axial cell-wall modulus of about $20 \mathrm{GPa}$. They observed that the cell wall cross-sections of chemically isolated fibers shrank much more transversely than the mechanically separated fibers.

The experiments have shown that the MFA and elastic modulus have a negative correlation with each other. So the elastic modulus increases with the decrease of MFA [60]. Figure 1.13 shows the stress-strain curves of a single wood fiber according to the different microfibril angles [61]. There is a plastic behavior for high microfibril angles, while the failer happens at $20 \%$ elongation of fibers. However, for the low microfibril angles, the curve rising almost sharply and linearly at a $5 \%$ elongation of fibers. This concludes that tensile strength and Young's modulus decrease with the increase in the microfibril angle.

Furthermore, combining micro-tensile tests with other techniques enabled researchers to see structural changes and material response at given stress and strain levels: For example, the failure mechanism of the single fibers identified by Scanning Electron Microscopy [62, 63]. Furthermore, polarized light microscopy facilitated the visualization of the distorted areas from the fibrillary cellulose alignment. This observation could help to understand the dislocations' role during the strain [63, 64]. Moreover, in situ X-ray experiments were conducted to see the variations in the crystal lattice of cellulose and microfibril angles (MFA) under stress [65, 66].

Bending of cantilever beams prepared with a focused ion beam (FIB) was one of the other methods for axial cell wall modulus measurement performed with Orso et al. [67]. The axial compressive strength and the failure mechanism of the secondary cell wall of spruce wood were studied by Adusumalli et al. They performed compression tests on the FIB machined micropillars and reported the yield strength values of approximately $160 \mathrm{MPa}$ [68]. They suggested that the deformation and failure of the S2 cell wall micropillars are caused by the out-of-plane bulging and shear bands formation, respectively.

In 1997, nanoindentation, a high-resolution mechanical test, was initiated to analyze the 


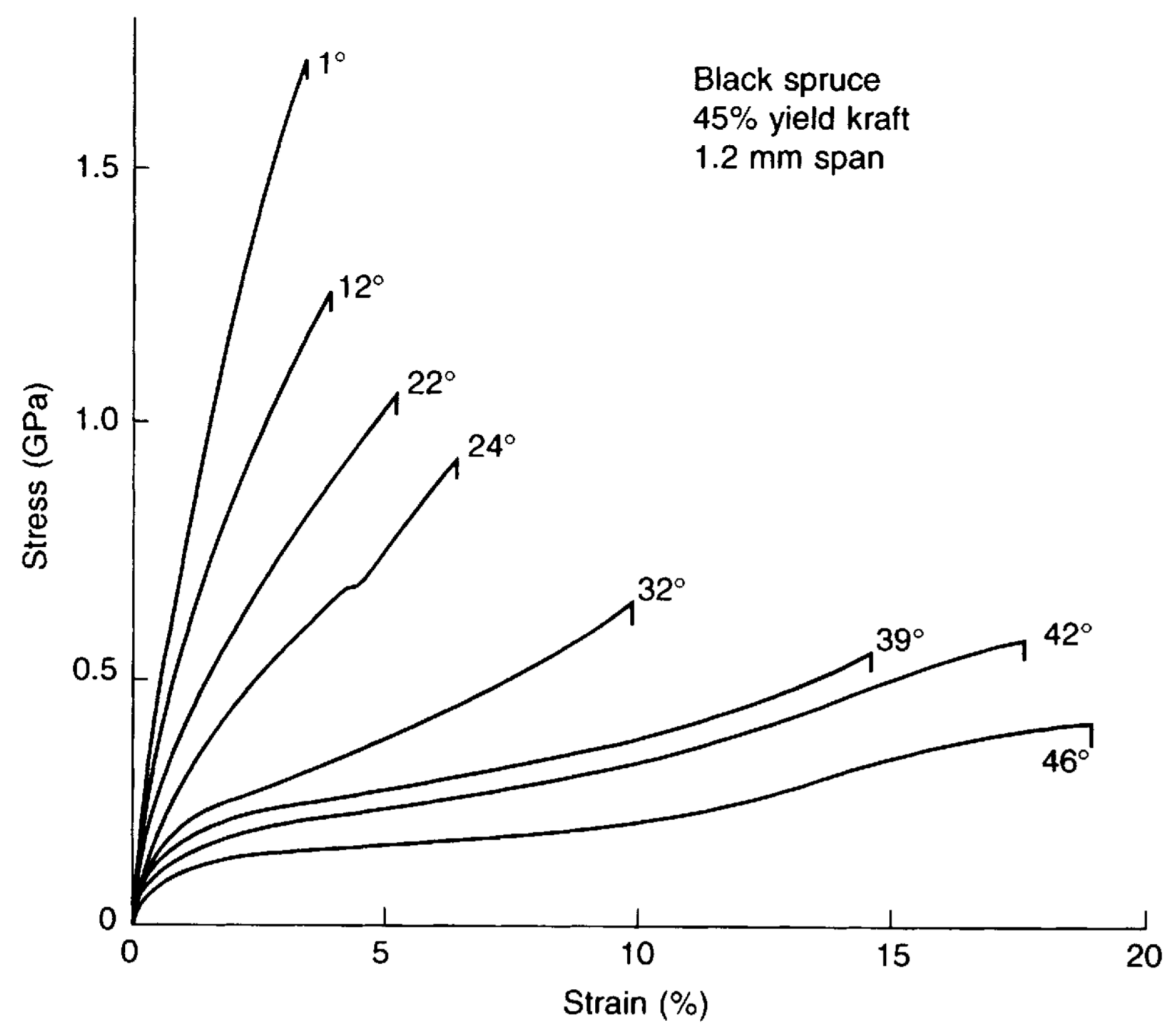

Figure 1.13: Stress-strain curve of the single wood pulp fibers with different microfibril angles [61]. Reprinted from T. Nishino [21], Copyright 2004, with permission from Elsevier.

wood cell wall by Wimmer et al. [69]. Nanoindentation performs approximately the same technique as hardness testing; however, the indenter's loading and displacement are tracked precisely during the experiment. This has the important advantage that, in addition to obtaining hardness values, it is also possible to extract parameters related to the material's elastic properties.

Measurements of the wood cell wall showed that earlywood and latewood's mechanical properties vary within the tree rings. However, a wide variety of mechanical properties was reported within a single tracheid as well [69].

The nanoindentation experiments of Gindl et al. [70] reported that the elastic modulus of cell walls with a large microfibril angle $(8.2 \mathrm{GPa})$ was consistent with the obtained value from tensile tests (10 GPa) [71]. However, it was shown that the elastic moduli of cell walls with small microfibril angles were underestimated in nanoindentation measurements 
$[69,70]$

In general, the measured values of direct methods for modulus of the wood cell wall, including tensile tests of isolated fibers [59], bending of a fiber cell wall [67], and nanoindentation measurements $[69,70,72,73,74]$, were lower than those obtained by extrapolation and theoretical calculations [51, 75, 76, 77]. It has been proposed that the inconsistency in the wood cell wall mechanical measurements is the consequence of the wood cell wall's anisotropic structure. Also, the nanoindentation elastic modulus of the wood cell wall reflects a combination of both axial and transverse properties, which causes an underestimation of the elastic modulus in the axial direction.

While many studies have focused on the mechanical properties of the wood cell wall, such as strength, stiffness, elastic modulus, and hardness, understanding the viscoelastic properties and damping mechanism of wood as a natural hybrid material is also essential. Viscoelasticity can be explained as a mixture of viscous and elastic characteristics. The loss factor is a measure of intrinsic damping, which is a valuable material property when structures vibrate [78]. The loss factor is the ratio of loss modulus to storage modulus representing the degree to which a material dissipates vibrational energy.

One of the important reasons that fiber-reinforced composites are different from other materials like metals is their damping mechanism. In the case of fiber-reinforced composites, the viscoelastic properties of matrix and fiber and damping due to the interface area have contributed to energy dissipation approaches. Interfacial displacement during vibration can cause debonding and friction in the interface field, which is described as a fundamental energy dissipation mode during vibration [79].

So far, the viscoelastic response of wood corresponding to different relative humidity conditions has been studied on bulk wood samples in different researches [80, 81, 82]. In the study of Meng et al., the viscoelastic parameters of the wood cell wall, measured with nanoindentation, showed a negative trend with increasing moisture contents [83].

In order to evaluate the properties of viscoelastic wood during humidity change cycles, Mukudai proposed a viscoelastic bending model based on the hypothesis that the looseness in the cell wall's microstructure between S1 and S2 layers caused a slippage between them during the drying process [84]. 
Regarding the nanoscale's mechanical properties, Atomic Force Microscopy is performed recently on the wood cell wall. This technique was used to measure the elastic modulus of cellulose fibrils in the bending process [85]. So far, the elastic properties in the wood cell wall were investigated using the Resonant Contact-AFM (RC-AFM) $[86,87,88]$ and AFM peak-force quantitative nanomechanical mapping (PF-QNM) methods [89, 90]. However, nanomechanical measurements are still challenging to be used to understand the individual polymers interactions and their effect on cell wall properties.

\subsection{Objectives of the study}

\subsubsection{Micromechanical measurements of the wood cell wall by Nanoindentation}

The present study aimed to analyze the elastic modulus, hardness, and viscoelastic properties of the wood cell wall under quasi-static and dynamic depth-sensing indentation measurements.

As the cell wall structure and chemical compositions play a crucial role in bearing the mechanical loads, different thermal modifications were applied to the wood samples to understand the effect of chemical compositional changes on the cell wall's mechanical function. The changes of the mechanical properties were examined before and after thermal treatments by nanoindentation.

The viscoelastic variations of the modified and unmodified wood samples were also studied in order to understand the intrinsic damping mechanism of wood.

\subsubsection{Nanoscale measurements of the wood cell wall by Atomic Force Microscopy}

The second part of this thesis aimed to present detailed information on the wood cell wall nanostructure. The spatial organization of cellulose fibrils in different secondary layers 
was investigated. The measurements were performed in radial and transverse sections of latewood tracheids to observe the fibril patterns from different perspectives.

This thesis is organized as follows:

In Chapter 2, the nanoindentation approach is used to calculate the micromechanical properties of pine and eucalyptus wood samples.

Chapter 3 investigates the ultrastructural arrangement of the wood cell wall using atomic force microscopy.

Chapter 4 of the thesis is devoted to the summary.

And the appendices have provided additional information for interested readers. 


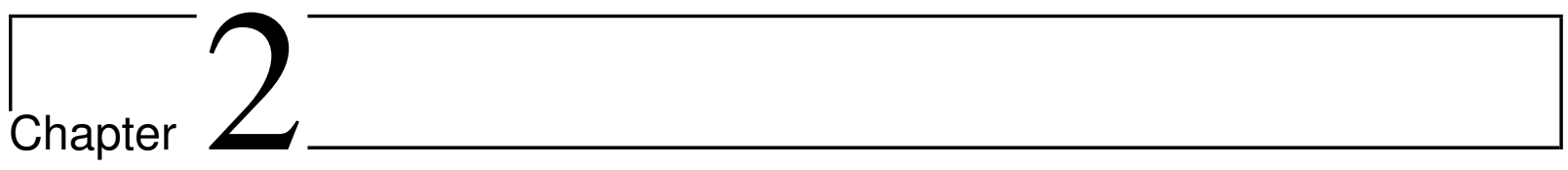

\section{Micromechanical measurements of the wood} cell wall

The remarkable mechanical properties of wood are derived from the hierarchical arrangement of its polymer components, accounting for macroscopic features such as tree rings, down to the nanoscale layers making up the walls of the wood cells. An important mechanical component of wood is the wood cell wall, which is composed of cellulose microfibrils embedded in a lignin matrix and anchored there by hemicellulose molecules. Here, we study the micromechanical properties of pine and eucalyptus cell walls, before and after various thermal treatments aimed at improving wood properties like dimensional stability and biological durability. Typical specimen preparation methods involving impregnation were avoided, so as not to change the wood cell wall properties. We detect significant variations in the local wood cell wall mechanical properties, which we show are well explained by local variations in polymer composition and MFA. Based on this, we introduce a measurement method that allows the intrinsic loss factor of the wood, unaffected by local composition and MFA, to be determined. We detect a clear decrease in loss factor and a modest increase in modulus and hardness as a result of the thermal treatments. We argue that the property changes due to thermal treatment are primarily explained by the loss of hemicellulose and the increase in the crystallinity of cellulose. 
WALL

\subsection{Introduction}

The mechanical properties of the wood cell wall as a polymer-based hierarchical composite are basically a combination of the heterogeneous polymeric composition's role at the molecular scale and the architecture of the laminated structure of the secondary layers at the nanometer scale. One of the long-standing goals of materials science has been to relate the macroscopic properties of composites to the composite architecture and the mechanical properties of the components. The major polymeric components of the cell wall are cellulose, hemicellulose, and lignin, which contribute to wood strength in different ways, as suggested in the Rowell and Winandy models [20]. They suggest that mechanical properties are primarily the function of chemical bond strengths, including covalent and hydrogen intrapolymer bonds and covalent and hydrogen interpolymer bonds [33]. Any treatments affecting these bond strengths, including the thermal treatments investigated in the current study, specifically affect the mechanical characteristics.

Micromechanical methods based on nanoindentation (NI) have been widely used to characterize mechanical properties of materials at micrometer length scales and were first applied to studies of wood and wood cell walls by Wimmer et al. [69] in 1997. The key benefit of this method is that it can provide quantitative values for hardness, modulus, and loss factor in micrometer resolution. Combined with macroscopic measurements of mechanical response and modeling of the specific architecture, micromechanical studies allow a quantitative understanding of wood cell wall properties and guidelines for developing bio-inspired materials. In addition to first illustrating the possibilities of high-resolution mechanical studies for investigating the properties of wood, the nanoindentation studies of Wimmer et al. [69] revealed axial hardness values of the cell walls as large as those of aluminum, and significant variations in hardness and modulus between earlywood and latewood and within single-cell walls. These variations were attributed to the well-known variations in cellulose microfibril angle, cellulose crystallinity, and lignin content with the position.

Since then, a number of NI-based studies have focused on relating local wood cell wall structure to local mechanical properties [91]. The study of Konnerth et al. [73] pointed out that the few degrees of cellulose fibers misalignment that could occur by specimen 
inclination during specimen preparation and also the differences of MFA in adjacent cell walls within one double cell wall could be the cause of the high variability in the elastic modulus of the wood cell wall observed by nanoindentation. Further, the measurements of Gindl et al. [74] on developing and fully lignified spruce tracheid indicated that an increase in the packing density and filling the spaces of the cell wall during lignification, and the increase of lignin content could cause the observed increase of elastic modulus and hardness of mature cell walls [74]. It was shown that MFA has a negative correlation with the elastic modulus. Furthermore, it was suggested that the compressive yielding of the wood-cell wall is a matrix-dominated process and mostly independent of MFA [70]. In the study of Wang et al. [92], the contribution of chemical compositions in the mechanical properties of the wood cell wall was investigated by nanoindentation. It was reported that the removal of hemicellulose and lignin in the wood cell wall caused $11.7 \%$ and $28.4 \%$ decrease in elastic modulus, respectively, and $14.8 \%$ and $30.4 \%$ decrease in hardness, respectively. Furthermore, it was stated that the elasticity of the cell wall could be affected by the lignin content, which is in agreement with Gindl et al. [70, 74].

The modulus and hardness values of pine and eucalyptus wood samples measured with nanoindentation in different literature works are presented in Table 2.1. It should be noted that there are wide variations in the properties of wood, either from the same species or within a single tree. So it is not surprising to see different values in different studies regarding the mechanical properties of the wood cell wall; although, they used all nanoindentation method for mechanical measurement.

Table 2.1: The reported values of pine and eucalyptus wood modulus and hardness, measured by nanoindentation in various studies.

\begin{tabular}{lccc}
\hline \hline & Modulus (GPa) & Hardness (GPa) & Ref. \\
\hline Eucalyptus species & 12.52 & 0.31 & {$[93]$} \\
& $16-19$ & $0.24-0.31$ & {$[94]$} \\
\hline Pine species & $12.7-17.9$ & $0.34-0.54$ & {$[95]$} \\
& 19 & 0.510 & {$[96]$} \\
& 18.8 & 0.43 & {$[97]$} \\
\hline \hline
\end{tabular}


WALL

Nanoindentation has also been widely used to obtain viscoelastic properties of polymerbased materials [98, 99, 100], in addition to their hardness and modulus. Studies in both the time domain (creep and stress relaxation) and the frequency domain (storage and loss moduli) have been performed and can be quantitatively related to each other under certain conditions [101].

Regarding the different nanoindentation methods, creep relaxation is a measure of strain under a constant stress level. Stress relaxation is a decrease in stress under constant strain. The dynamic indentation is a sinusoidal force that is applied to the specimen, and by monitoring the resulting strain response, the storage modulus (elastic response) and loss modulus (flow response) will determine [102]. In this regard, Maxwell and Kelvin-Voigt models have been established. The Maxwell model, which is suitable for viscoelastic liquid, has been defined as a purely elastic spring and a purely viscous dashpot in series [78, 102]. The Kelvin-Voigt model for a viscoelastic solid has been defined as the pure elastic spring and the pure viscous dashpot in parallel. However, since the Voigt model cannot describe the stress relaxation experiment [102], in NI stress relaxation methods, to have a better approximation, a combination of various dashpots and springs as the Burgers model (spring and dashpot added in series to the Voigt model) is used. So creep relaxation and stress relaxation analysis in most of the studies of viscoelasticity of the wood cell walls were based on the Burgers model [83, 92]. In comparison, the typical model for the dynamic loading method is the Generalized Voigt model with two components [101, 102].

According to published reports, the viscoelastic mechanism of fiber-reinforced composites is different from conventional monolithic materials. In fiber-reinforced composites, not only the viscoelastic characteristics of matrix and fiber but also the damping due to the interface region have a significant contribution to energy dissipation. Interfacial displacements may cause debonding and friction in the interface region, which have been identified as the fundamental energy dissipation mode during vibration [79].

More recently, dynamic nanoindentation methods have been applied to gaining insight into the viscoelastic properties of wood cell walls [83, 92, 103, 104, 105]. As expected, the viscosity of wood is found to decrease with moisture content [83], while in another study, where the different polymer components were selectively extracted from wood, it was concluded that the hemicellulose dominates the viscous response of the wood cell 
walls [92]. In the study of Zhang et al. [103], dynamic properties of bulk wood specimens were investigated using free-free flexural vibration tests. The specific dynamic modulus $\mathrm{E}^{\prime} / \rho$ ranged from 16 to $35 \mathrm{GPa}$, and the damping coefficient (measured by the logarithmic decrease of amplitude after stopping the excitation) ranged from $5 \times 10^{-3}$ to $9.5 \times 10^{-3}$. They reported that the observed decrease of $\mathrm{E}^{\prime} / \rho$ was correlated with an increasing MFA. However, the damping coefficient variation could be partly explained by MFA and partly by chemical constituent variations.

In the current study, we investigate the static and dynamic mechanical properties of pine and eucalyptus wood cell walls, both in air-dried and thermally modified states. We use the dynamic NI method, which involves superimposing an oscillatory load with a frequency of $75 \mathrm{~Hz}$ during slow loading of the NI tip and monitoring the amplitude and phase lag of the displacement response. We record hardness, storage modulus, loss modulus, and loss factor as a function of position in the wood cell walls and find that in contrast to all other measured properties, the loss factor is relatively insensitive to the natural local variations in cell wall properties. It, therefore, offers a sensitive metric to detect changes due to externally imposed fields such as thermal modification. We use it to investigate changes at the wood cell wall level due to environmentally friendly thermal treatments, which are known to improve dimensional stability and biological durability.

\subsection{Materials and Methods}

\subsubsection{Specimen preparation and characterization}

This study was performed on transverse sections of pine and eucalyptus sapwood, as representative of softwoods and hardwoods, respectively. The pine latewood cell wall thickness was $6.66 \pm 1.53 \mu \mathrm{m}$, and the eucalyptus latewood cell wall thickness was $1.85 \pm 0.35 \mu \mathrm{m}$.

The relative content of the wood polymer constituents in both untreated and thermally treated states was obtained by chemical analysis [106] after first removing and weighing solvent extractives [107]. Holocellulose was measured based on the separation of the lignin by sodium chloride [108]. The relative content of cellulose was measured according to the 
WALL

TAPPI test method 203 [109], where cellulose was separated from the hemicellulose using a sodium hydroxide solution. The relative content of lignin was obtained using the NREL (National Renewable Energy Laboratory) standard determination [110].
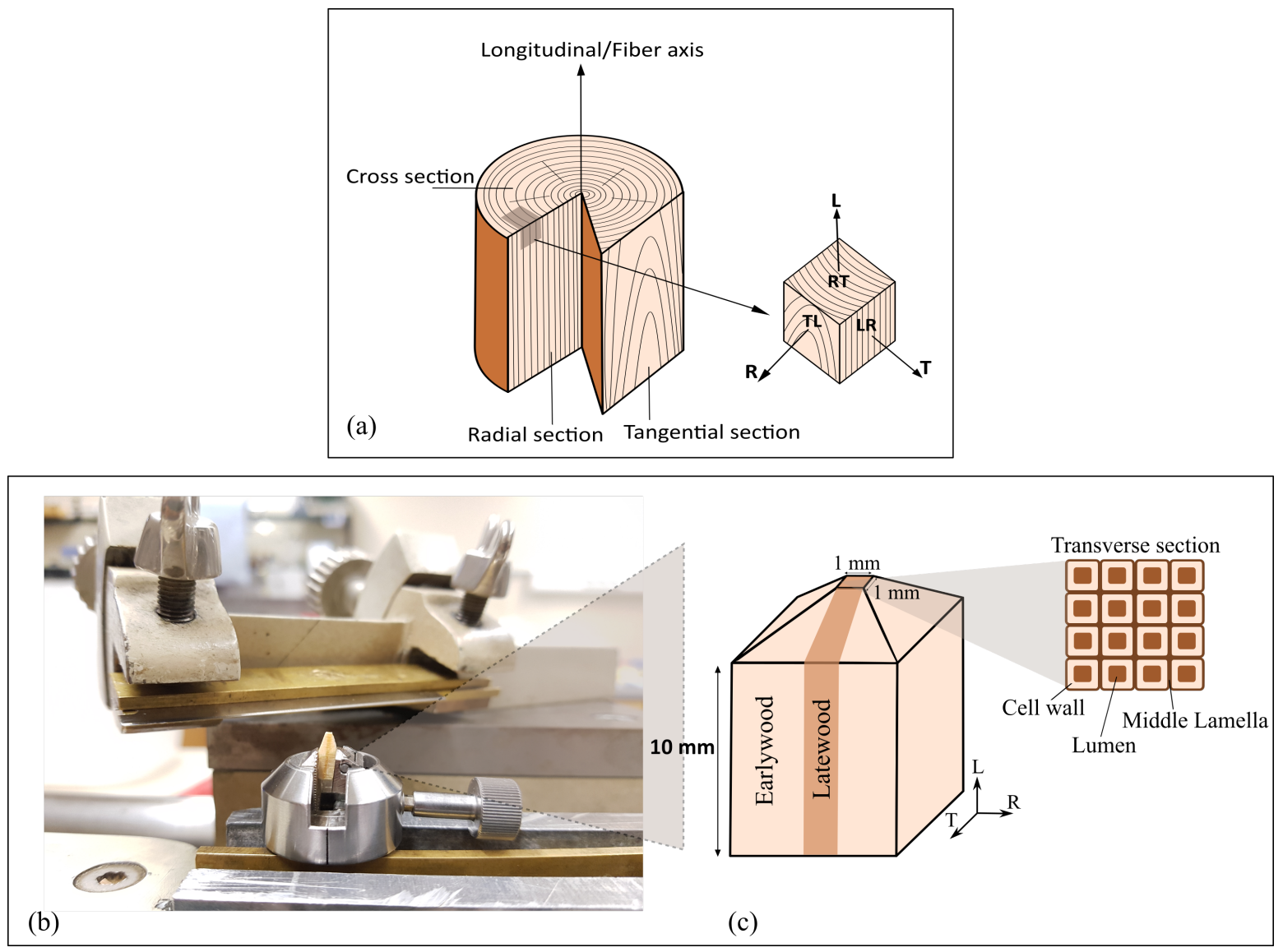

Figure 2.1: (a) Schematic illustration of the tree trunk and corresponding radial, tangential and longitudinal directions, (b) the uninfiltrated pine wood specimen located in the sledge microtome, (c) schematic picture of the prepared pyramid specimen with the smooth transverse surface positioned in the latewood.

A smooth transverse section of the wood samples is required for reproducible nanoindentation measurements. This is widely achieved by embedding the specimens and then slicing them with a microtome. Effects of embedding medium on the hardness and modulus of the wood cell wall have been studied by Meng et al. [111]. It was stated that the mean value of Young's modulus and the hardness of loblolly pine embedded with epoxy resin were higher than of the reference samples, which were not embedded. Therefore in the current study, in order to prevent changing the mechanical properties of the wood cell wall, all forms 
of embedding media and infiltration were avoided. Instead, we followed an infiltrationfree method established by Jakes et al. [96], which allows smooth sections of unembedded wood to be prepared without buckling and crushing the wood cells. Pyramid-shaped wood specimens with their apexes pointing in the longitudinal direction and positioned in the latewood are prepared by cutting with a razor blade, as shown in Figure 2.1. The apexes are brushed gently with water, and following this, $10 \mu \mathrm{m}$ slices are removed from the apexes of the pyramids using a Sartorius 31A30 sledge microtome, equipped with a disposable steel knife, to produce smooth transverse surfaces of latewood with approximately $1 \times 1 \mathrm{~mm}^{2}$ areas.

The root mean square (RMS) roughness of the prepared surfaces is between 4-10 nm, as obtained from atomic force microscopy (AFM) measurements of $2 \times 2 \mu \mathrm{m}^{2}$ areas (Figure 2.2).

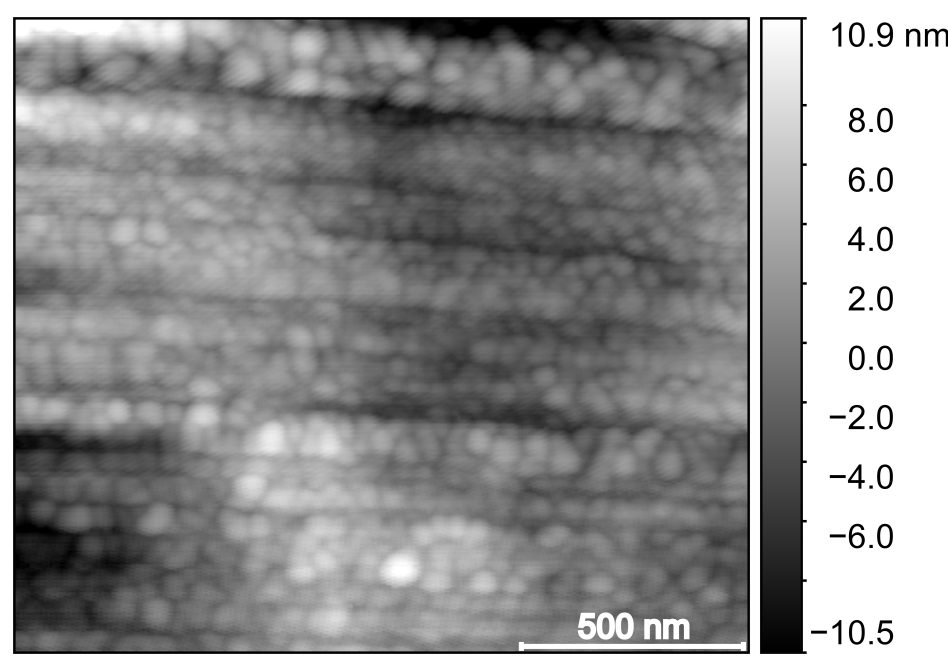

Figure 2.2: Topography image of the S2 layer provided with Atomic Force Microscopy.

The general procedure used for micromechanical testing consisted of preparing a pyramid specimen with a smooth transverse surface, air-drying at room temperature for several days, equilibrating the moisture content in the nanoindentation chamber, performing mechanical tests, applying thermal treatment, equilibrating moisture content again, and performing mechanical tests once more. The climate chamber was held at $65 \% \mathrm{RH}$ and $21^{\circ} \mathrm{C}$, to imitate ambient conditions, and resulted in moisture contents of 9\%-12\%. At least three specimens were prepared and tested for each different thermal treatment.

The moisture contents were determined by comparing weights of cube samples of pine and 
WALL

eucalyptus wood before and after thermal modifications with their oven-dry weights.

Thermal treatments were carried out by Maximilian Wentzel at the Institute of Wood Biology and Wood Products, as were the chemical and mass analyses and moisture content measurements [106]. The thermal treatments were performed in a laboratory-scale closed reactor autoclave at $160^{\circ} \mathrm{C}$ for 3 hours under different pressures and relative humidities (Table 2.2) using the following four steps [106, 112, 113]:

1. $50 \mathrm{~min}$ hold at room temperature and a pressure of less than $17 \mathrm{kPa}$,

2. temperature increase at a rate of $12^{\circ} \mathrm{C}$ per hour to the hold temperature of $160^{\circ} \mathrm{C}$,

3. hold at $160^{\circ} \mathrm{C}$ for 3 hours after introducing pre-heated pressurized steam at selected pressures and relative humidities (Table 2.2),

4. cool at a rate of $20^{\circ} \mathrm{C}$ per hour down to $65^{\circ} \mathrm{C}$.

Table 2.2: Different thermal treatments applied to the pine/eucalyptus wood samples and the resultant moisture content (MC) and corrected mass loss (CML). Maximilian Wentzel carried out thermal treatments, mass analyses and moisture content measurements at the Institute of Wood Biology and Wood Products, University of Göttingen.

\begin{tabular}{lcccccc}
\hline \hline Treatment & $\begin{array}{c}\text { Hold temp. } \\
\left({ }^{\circ} \mathrm{C}\right)\end{array}$ & $\begin{array}{c}\text { Pressure } \\
(\text { bar })\end{array}$ & $\begin{array}{c}\text { RH } \\
(\%)\end{array}$ & $\begin{array}{c}\text { Hold time } \\
(\mathrm{h})\end{array}$ & $\begin{array}{c}\text { MC } \\
(\%)\end{array}$ & $\begin{array}{c}\text { CML } \\
(\%)\end{array}$ \\
\hline Unmodified ("Ref") & - & - & - & - & $9.51 / 9.91$ & - \\
"1 bar" & 160 & 1 & 15 & 3 & $3.76 / 2.64$ & $1.09 / 1.43$ \\
"3 bar" & 160 & 3 & 50 & 3 & $4.16 / 3.36$ & $3.44 / 3.78$ \\
"6 bar" & 160 & 6 & 100 & 3 & $4.61 / 4.48$ & $8.76 / 16.26$ \\
\hline \hline
\end{tabular}

The average equilibrium moisture content at $20^{\circ} \mathrm{C}$ and $65 \% \mathrm{RH}$ after the different treatments are listed in Table 2.2 and shown in Figure 2.3. The treated specimens are clearly less hygroscopic than the untreated wood but become more hygroscopic with increasing $\mathrm{RH}$ of the treatment. This is entirely consistent with previous studies on beech wood [114], where the effect is explained by avoiding immoderate drying, prevention of complete closure of 
microvoids, and realignments of amorphous polymers during the cooling stage under high $\mathrm{RH}$ conditions. Indeed as water acts as a softener agent and minimizes the Tg of amorphous cell wall polymers, during the cooling phase under high relative humidity conditions, the amorphous polymers are more in a soft and rubbery state and have more time to reorient their structures. This reorientation prevents the formation of irreversible hydrogen bonds and increases the access ability of the hydroxyl group to attract water after the cooling stage.

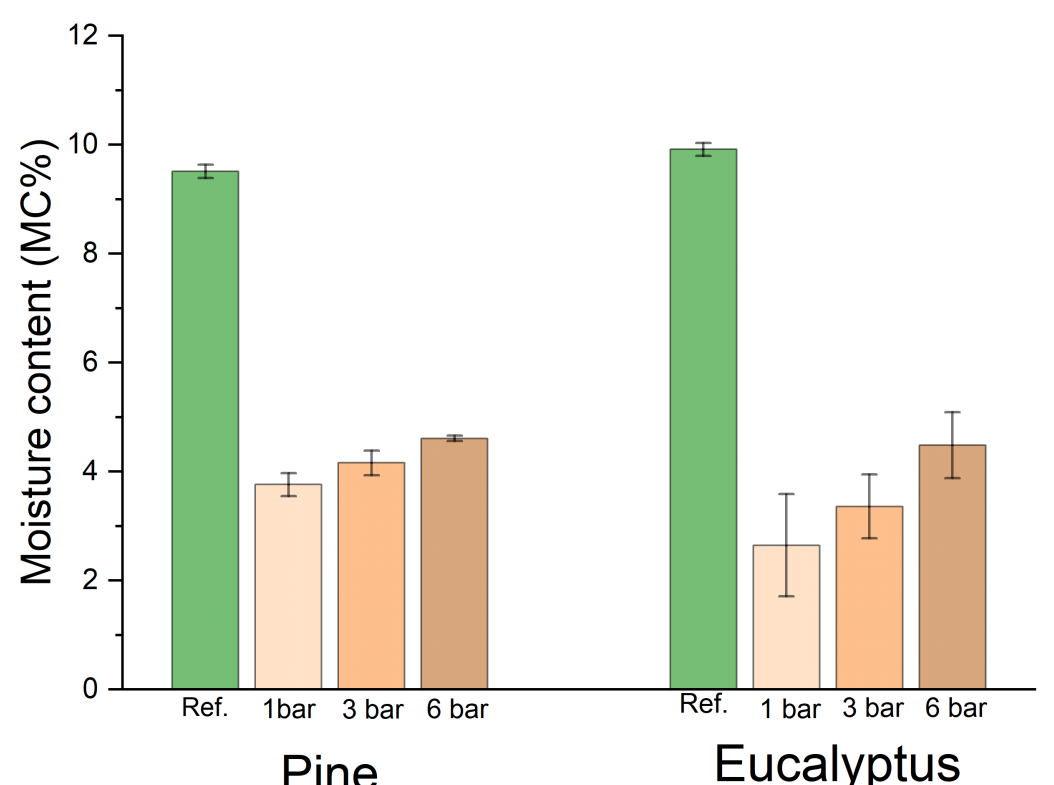

Figure 2.3: The moisture content in pine and eucalyptus wood samples before and after thermal treatments, relative to the oven-dry state.

The less hygroscopicity after thermal modification was observed in the study of Kocaefe et al. [115] as well, where the effect is explained by the preferential loss of hemicellulose, which is strongly hygroscopic.

The average corrected mass losses (CML) due to each treatment are listed in Table 2.1 and shown in Figure 2.4. The corrected masses have had the mass of solvent extractives subtracted out [107] so that the CML is a measure of carbohydrate degradation due to thermal treatment and does not include effects of extractive evaporation. At temperatures below $180^{\circ} \mathrm{C}$, the degradation is usually predominately attributed to hemicellulose reac- 

WALL

tions [115, 116]. As seen previously for beech wood [114, 117], the extent of mass loss for both the pine and eucalyptus increases with increased pressure and $\mathrm{RH}$ of the treatment. After treatment with the highest pressures and humidity (6 bar and 100\% RH), the eucalyptus wood samples show considerably more mass loss than the pine wood samples.

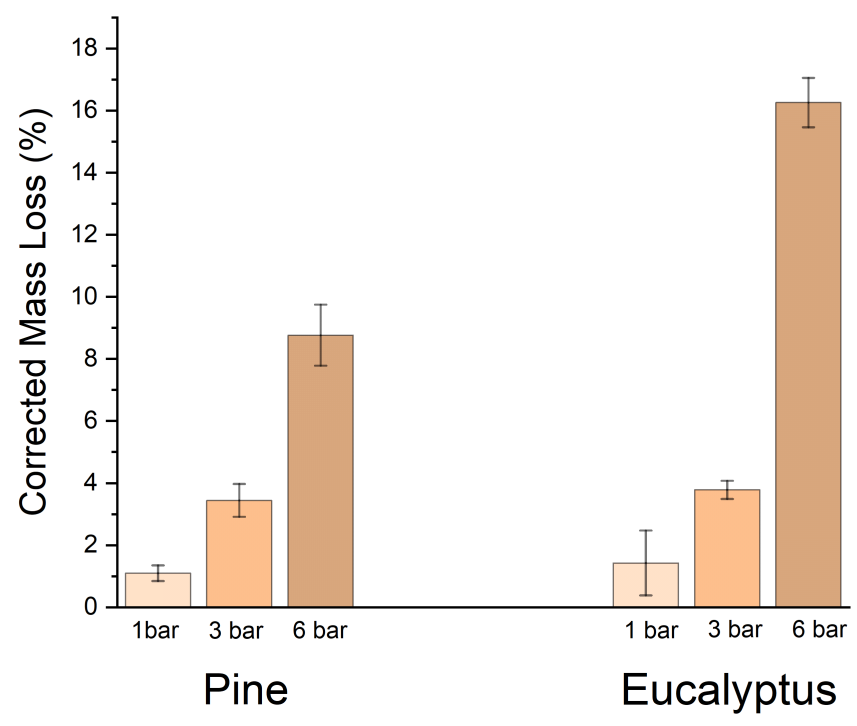

Figure 2.4: Corrected mass loss in pine wood and eucalyptus wood due to thermal modification.

The relative contents of wood polymer constituents for the different thermal modifications are shown as a percentage of the initial dry mass of each sample in Figure 2.5. It is seen that the relative cellulose and hemicellulose contents tend to decrease with pressure and $\mathrm{RH}$ of the treatment, while the relative lignin and extractive contents increase. This is consistent with the idea that hemicellulose and part of the amorphous cellulose are most strongly degraded during thermal treatment [118]. The significant loss of eucalyptus hemicellulose after treatment with the highest pressures and humidity ( 6 bar and 100\% RH) is consistent with the large mass loss for this treatment. 


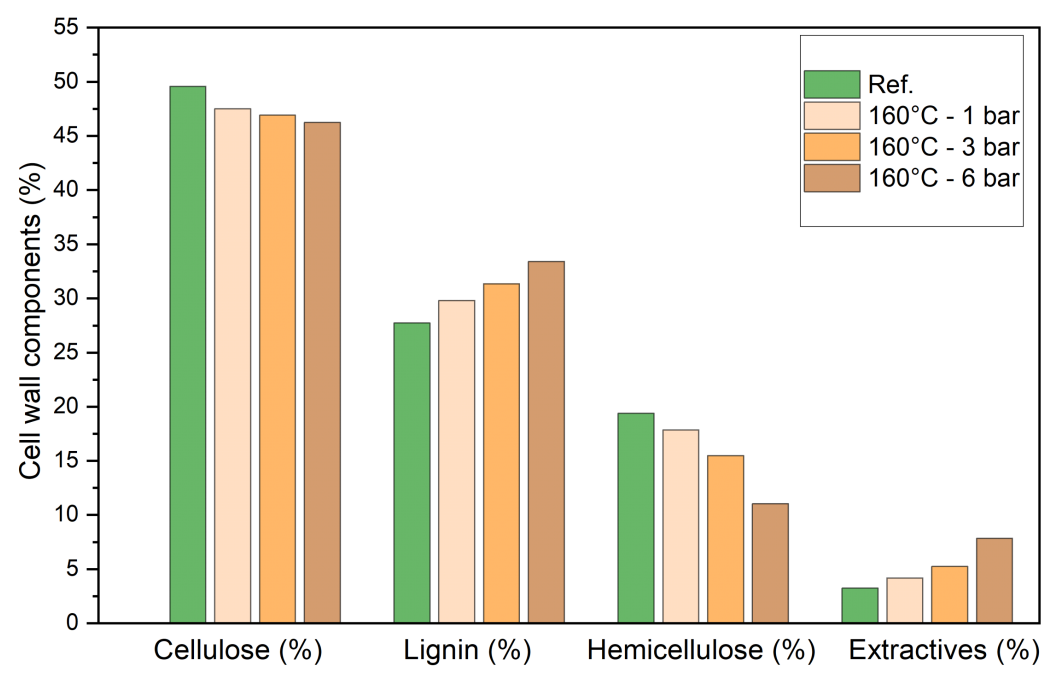

a)

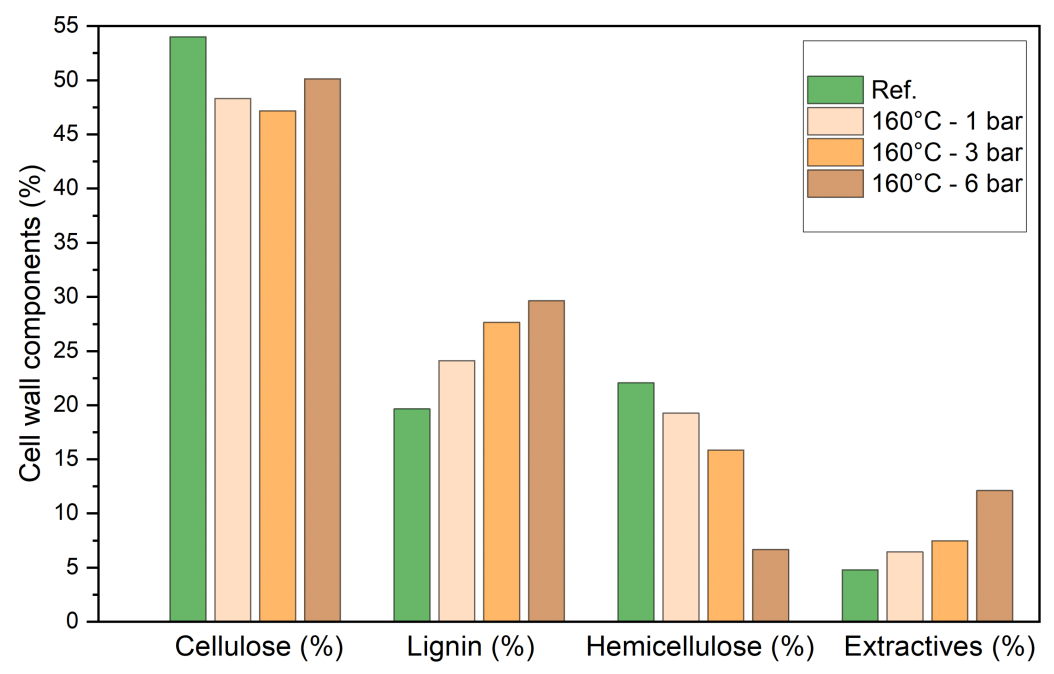

b)

Figure 2.5: The relative weight content of wood polymer constituents for the different thermal modifications of (a) pine wood and (b) eucalyptus wood. The data were provided by Maximilian Wentzel at the Institute of Wood Biology and Wood Products, University of Göttingen.

\subsubsection{Nanoindentation measurement methods}

A Nanoindenter (G200, Nano Instrument, MTS, USA) equipped with a diamond Berkovich tip with an approximate $100 \mathrm{~nm}$ tip radius was used to determine the local mechanical 
WALL

properties of the latewood cell walls under ambient conditions $\left(21^{\circ} \mathrm{C}, 65 \%\right.$ relative humidity). Hardness and elastic modulus are obtained from standard nanoindentation loaddisplacement (P-h) curves, which are analyzed with the Oliver and Pharr method [119]. The method is based on comparing the loading and unloading segments of a single indent, where the loading segment is assumed to include both elastic and plastic deformation, while only elastic recovery occurs during unloading. The hardness at any given depth is defined as:

$$
H=\frac{P}{A}
$$

where $\mathrm{P}$ is the load, and $\mathrm{A}$ is the projected contact area. The value of $\mathrm{A}$ as a function of depth is obtained from calibration on a fused silica reference sample. It should be mentioned that the primary aim of hardness testing is to quantify a material's resistance to plastic deformation. In a perfectly rigid material, since all deformation is irreversible, no elastic energy is subjected in the deformed material. In this category, indentation hardness is a reliable indicator of a material's resistance to irreversible deformation. In the material with the infinite yielding stress, all deformation is raised reversibly, so that the contact area and hardness measurement is fully determined based on the material elastic properties and the indenter geometry. As a consequence, for the wide range of materials that come between these two categories, indentation hardness is calculated concerning the material's resistance to both irreversible and reversible deformation [145].

The effective elastic modulus, defined as:

$$
\frac{1}{E_{e f f}}=\frac{1-\vartheta^{2}}{E}+\frac{1-\vartheta_{i}^{2}}{E_{i}}
$$

takes into account elastic displacements that occur in both the diamond tip (with Young's modulus $E_{i}=1141 \mathrm{GPa}$ and Poisson ratio $\left.\vartheta_{i}=0.07\right)[119]$ and the specimen $(E$ and $\vartheta)$. For wood cell walls, the modulus $E$ is highly anisotropic, while a value of $\vartheta=0.3$ is often assumed [69]. The effective elastic modulus can be obtained from the initial unloading 
slope, or contact stiffness, $S=d P / d h$ according to:

$$
E_{e f f}=\frac{S}{2 \beta} \sqrt{\frac{\pi}{A}}
$$

where $\beta=1.034$ for Berkovich tips [119].

Nanoindentation tests were performed using a 5 second load controlled ramp-up to $250 \mu \mathrm{N}$, followed by a 10 second load hold, and then a 5 second controlled unload. The 10 second hold was necessary to allow for the further sink-in of the tip due to the time-dependent response of the wood, which because of its viscoelasticity, shows a lag between the applied force and the response of the material. Further increases in the hold time did not lead to a measurable change in the initial unloading slope, indicating that 10 second is sufficient to allow all measurable creep displacements to occur.

The time-dependent response of the wood cell walls was investigated using dynamic nanoindentation. In this case, a small oscillatory force $F(t)=F_{0} e^{i \omega t}$ with a frequency of 75 $\mathrm{Hz}$ is superimposed on the load-displacement cycle, and both the displacement amplitude $Z_{0}$ and phase lag $\varphi$ of the resultant oscillatory displacement $Z(t)=Z_{0} e^{i(\omega t-\varphi)}$ are recorded [101, 120, 121] (see Figure 2.6).

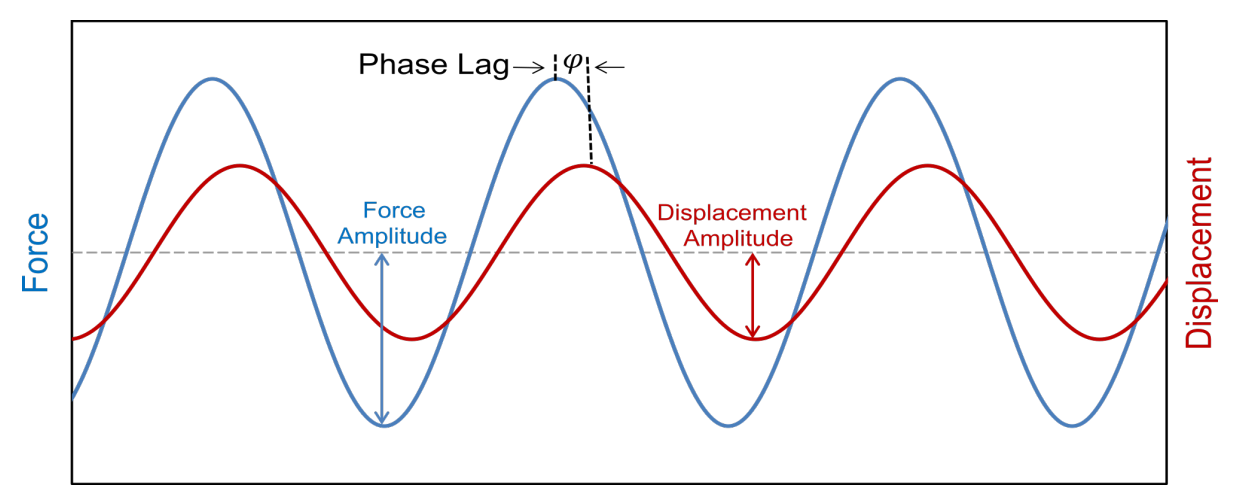

Figure 2.6: A representative figure of the sinusoidal force, applied to a sample and its response with the phase angle of $\varphi$.

The mechanical response of a viscoelastic material to an oscillatory load is characterized by the complex modulus [78]:

$$
E^{*}=E^{\prime}+i E^{\prime \prime}
$$


WALL

where the storage modulus $E^{\prime}$ is in phase with the load and represents the material's capacity to store energy, while the loss modulus $E^{\prime \prime}$ is out of phase with the load and characterizes the material's capacity to dissipate energy. The ratio of the loss modulus to the storage modulus is called the loss factor, $E^{\prime \prime} / E^{\prime}=\tan (\varphi)$, and is used as the measure of damping in viscoelastic materials.

The storage and loss moduli are typically analyzed using a Voigt spring and dashpot model for the nanoindenter and specimen [78, 101, 122, 123], as shown in Figure 2.7. It is assumed here that the nanoindenter frame is infinitely stiff, which is reasonable when working with relatively complaint materials such as wood. The equations of motion for the free hanging and contact geometries are also listed in Figure 2.7 and can be used to obtain the contact stiffness $K_{c}$ and contact damping $D_{c}$ of the specimen,

$$
\begin{aligned}
K_{c} & =\frac{F_{0}}{Z_{0}} \cos \varphi(\text { contact })-\frac{F_{0}}{Z_{0}} \cos \varphi(\text { no }- \text { contact }) \\
D_{c} \omega & =\frac{F_{0}}{Z_{0}} \sin \varphi(\text { contact })-\frac{F_{0}}{Z_{0}} \sin \varphi(\text { no }- \text { contact })
\end{aligned}
$$

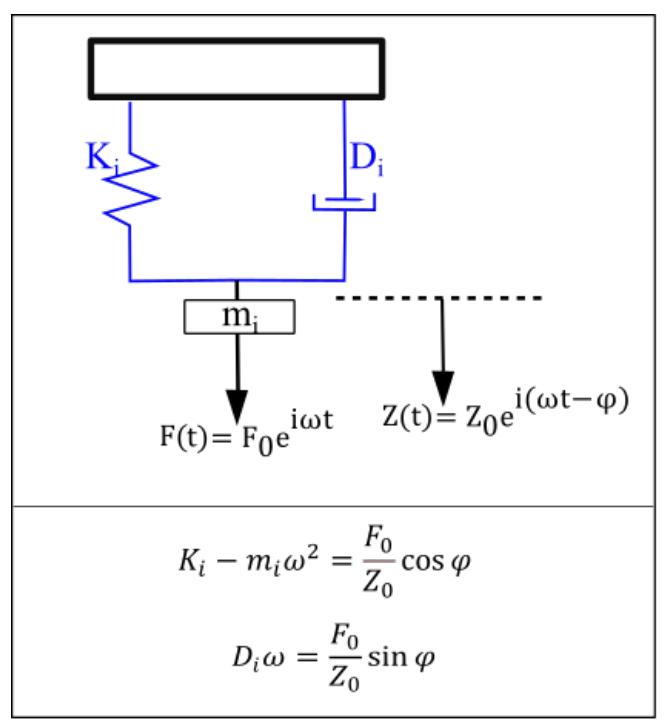

(a) Free hanging

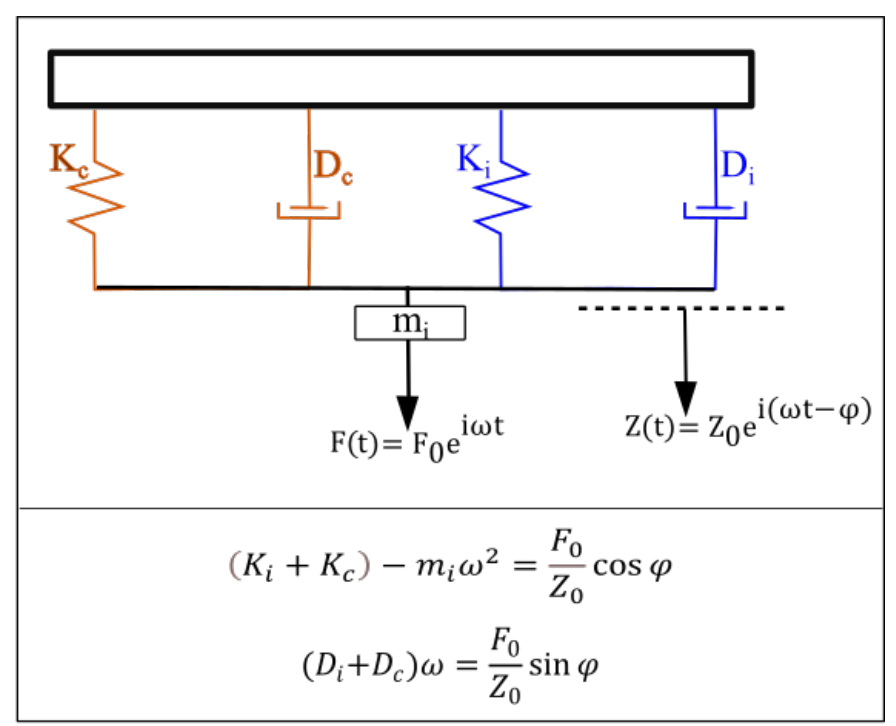

(b) Contact

Figure 2.7: A simple harmonic oscillator model for the nanoindenter machine (a) without and (b) with contact to a viscoelastic specimen. The resultant equations of motion are also included [122, 123]. 
Once the contact stiffness and damping are determined from the free-hanging and contact measurements, values for the storage modulus, loss modulus, and loss factor can be are calculated by analogy with the case for static indentation derived above (Eq. 2.3) [122]:

$$
\begin{array}{r}
E^{\prime}=\frac{\sqrt{\pi}}{2 \beta} \frac{K_{c}}{\sqrt{A}} \\
E^{\prime \prime}=\frac{\sqrt{\pi}}{2 \beta} \frac{\omega D_{c}}{\sqrt{A}} \\
\tan \varphi=\frac{E^{\prime \prime}}{E^{\prime}}=\frac{\omega D_{c}}{K_{c}}
\end{array}
$$

One of the beneficial aspects of using dynamic indentation testing is precise surface detection through surface contact stiffness, which increases abruptly when the surface is reached [124]. Figure 2.8(a) shows typical contact stiffness values while approaching the pine wood cell wall surface. The surface find criterion is a stiffness increase of more than $150 \mathrm{~N} / \mathrm{m}$, allowing surface detection to within $\pm 1 \mathrm{~nm}$. The method also allows surface roughness or particles to easily be detected, Figure 2.8(b), allowing these indents to be deleted from the results for further analysis.

After selecting the tests with a clear surface find, the hardness (Eq. 2.1) and storage and loss moduli (Eq. 2.6a) are calculated and plotted as a function of indentation depth. Typical results are shown in Figure 2.9. The increase, subsequent decrease, and leveling off of all three quantities as a function of depth is characteristic of nanoindentation and attributed to size effects [125]. Typically, values are recorded for a selected indentation depth range which represents a compromise between needing a small interaction volume to probe the heterogeneous properties of wood and reaching a sufficient depth to obtain a fairly constant value. In our case, we average and record values for indent depths between $100 \mathrm{~nm}$ and $200 \mathrm{~nm}$.

The variations in the hardness and moduli for the different indents in Figure 2.9 are not uncommon for nanoindentation and are attributed to local variations in material properties and in the contact area. The actual contact area will differ from the contact area calibrated on fused silica due to specimen surface roughness, inaccuracies in the surface find, and the local variations in material properties, which can lead to differences in sink-in and pile-up. Given that the Root Mean Square (RMS) surface roughness is less than $10 \mathrm{~nm}$ and the 


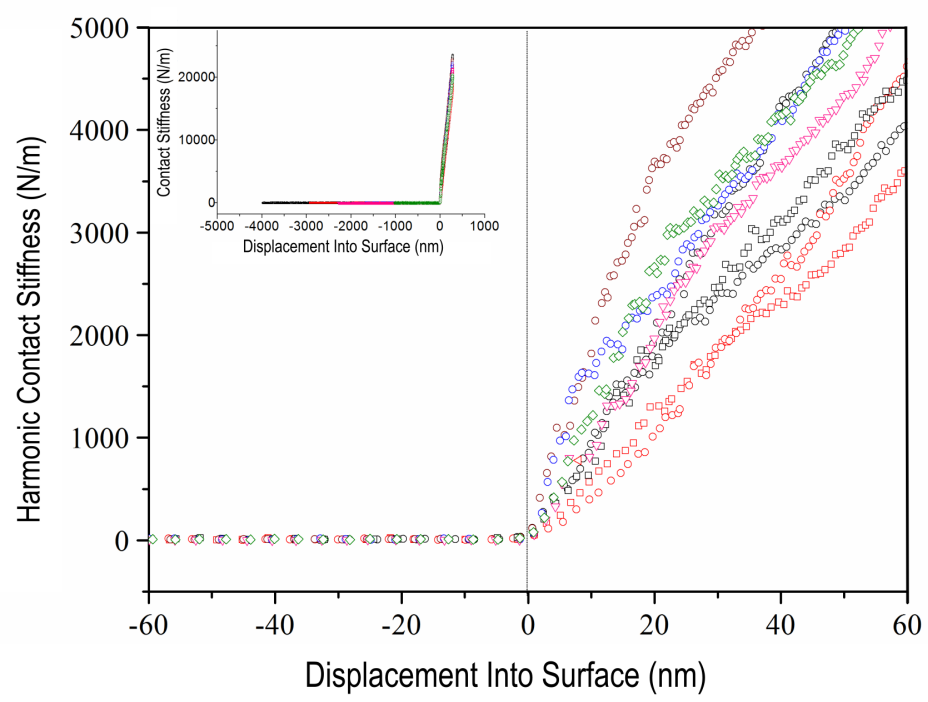

(a)

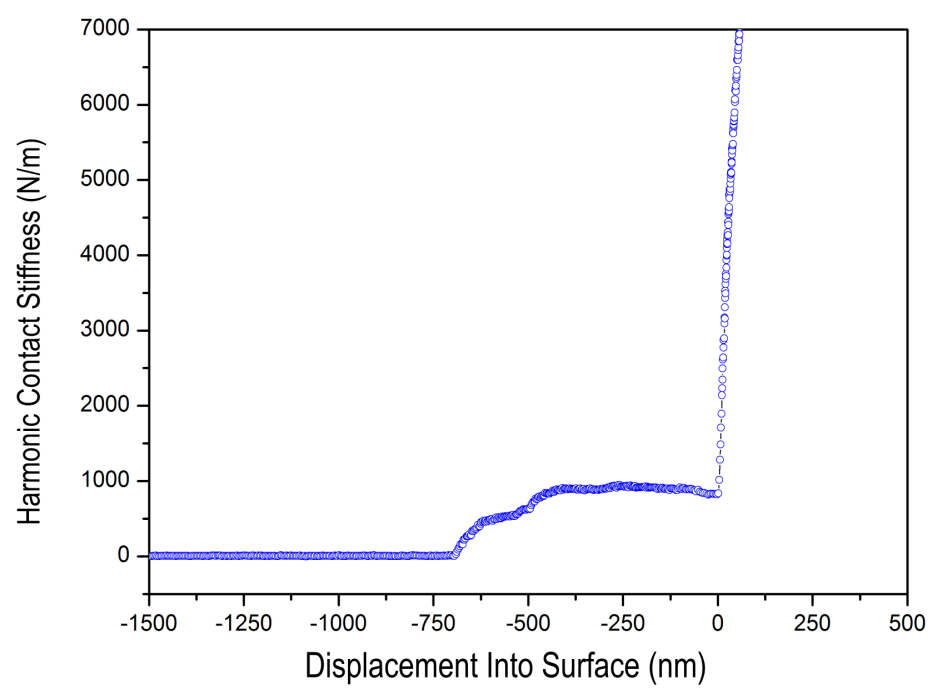

(b)

Figure 2.8: Surface detection with contact stiffness (a) when there is a smooth surface and (b) when the indenter meets roughness or particles.

surface find is precise to within a few $\mathrm{nm}$, it seems likely that the variations observed in Figure 2.9 result from local variations in material properties. These will be discussed later in more detail.

We note that the slightly more considerable variation in the hardness than in the storage 


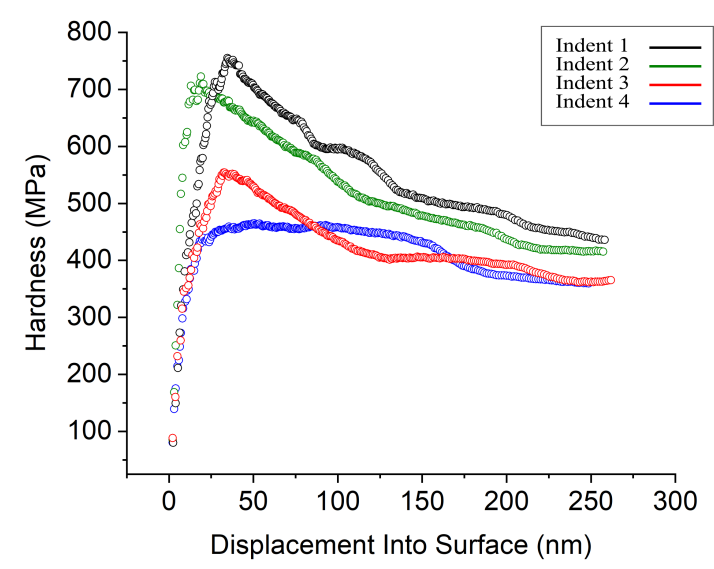

(a)

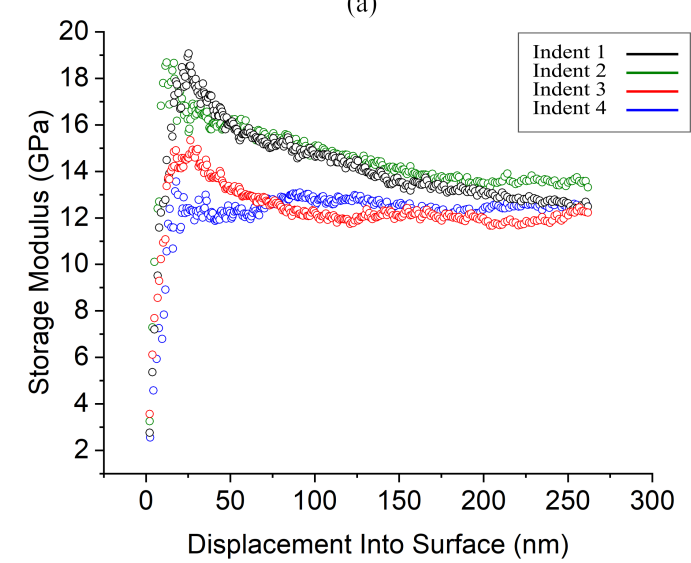

(b)

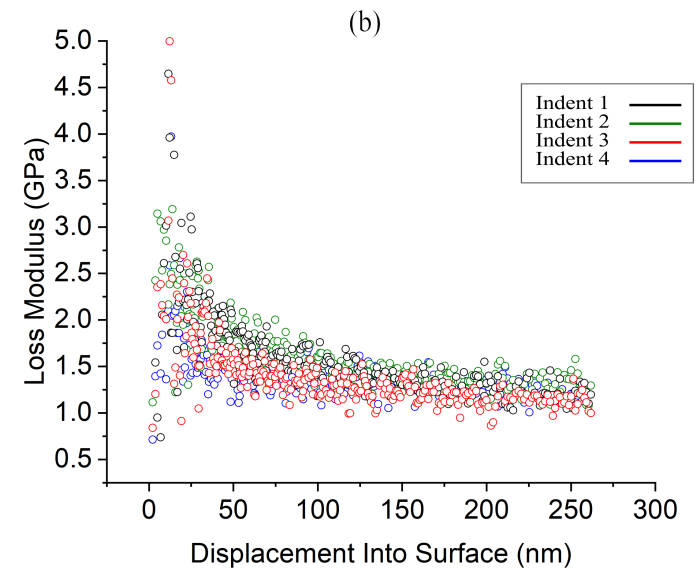

(c)

Figure 2.9: (a) Hardness, (b) storage modulus, and c) loss modulus as a function of depth for four representative indents into pine wood cell walls.

modulus may relate to the different elastic and plastic interaction volumes in the material. According to the widely accepted cavity model [126] shown in Figure 2.10, the material 


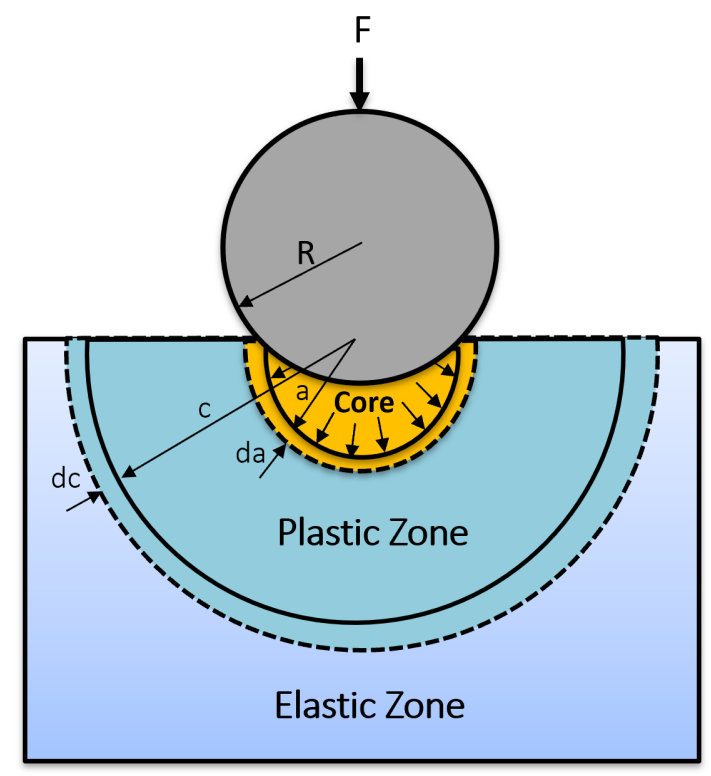

Figure 2.10: Cavity model of an elastic-plastic indentation by a sphere [126].

response under the indenter can be divided into a core zone $(\mathrm{r}<\mathrm{a})$, a plastic zone $(\mathrm{a}<$ $\mathrm{r}<\mathrm{c}$ ), and an elastic zone $(\mathrm{r}>\mathrm{c})$. Thus, the plastic response of a material, which is measured by the hardness, comes from a smaller volume than the elastic response. For material with heterogeneities on the length scale of the interaction volumes, the plastic response is expected to show larger variability than the elastic response. For Berkovich indents with depths between 100 and $200 \mathrm{~nm}$, plastic interaction volumes with dimensions of 1-2 $\mu \mathrm{m}$ and significantly broader elastic interaction volumes can be expected [127, 128]. Thus, material heterogeneities on the $\mu \mathrm{m}$ length scale will lead to the large variability in the hardness but will tend to be averaged out in the elastic response.

\subsubsection{Positioning Nanoindents in the cell walls}

The layered structure of the wood cell walls, with variations in composition and MFA, as well as the lumen, middle lamella, rays, and pits, all cause variations in the cell wall mechanical response. Since the primary goal of this study is to detect changes in mechanical response due to thermal treatments, it is necessary to find positions in the axial crosssections of wood cell walls that show the highest reproducibility. For this purpose, static 

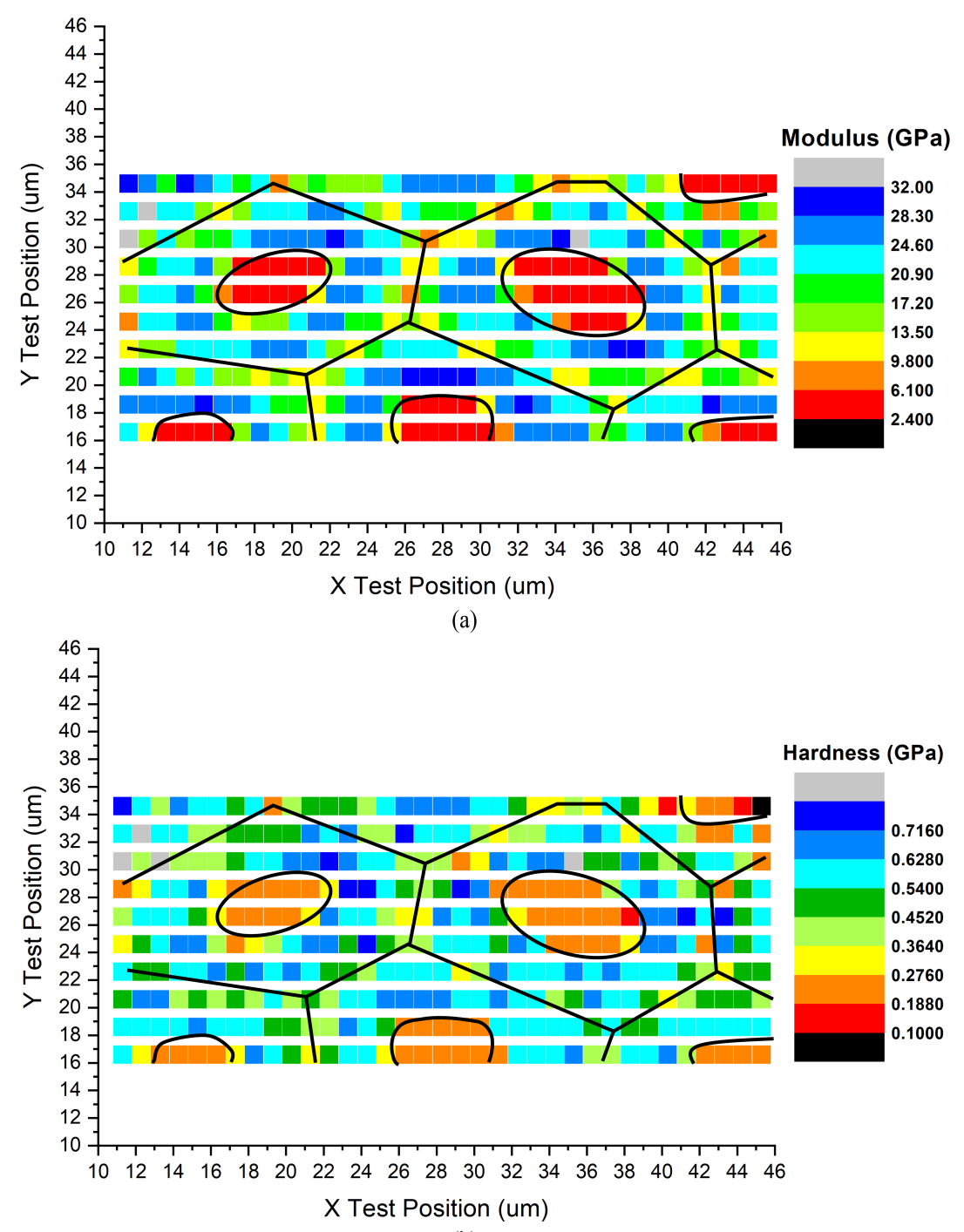

(b)

Figure 2.11: (a) Elastic modulus map and (b) hardness map obtained from static indentation arrays of a resin-embedded axial cross-section of beech wood. The black lines show the positions of the resin-filled lumen and the middle lamella.

indentation tests were performed on microtome prepared surfaces of epoxy resin-embedded beech wood cell walls, to map the heterogeneous response and identify optimal positions for obtaining reproducible results. Beech wood has large cells, making it an ideal material for studying the effects of indent positioning. An array of indents with a maximum load of $50 \mu \mathrm{N}$, and an indent depth of up to $60 \mathrm{~nm}$, was performed with spacings of 1 and $2 \mu \mathrm{m}$, 

WALL

in the $\mathrm{x}$ and $\mathrm{y}$ directions, respectively. The results were used to create a map of the elastic modulus obtained from the unloading curves, and a map of the hardness (Figure 2.11). The cell structure taken from optical microscopy images of the specimen has been sketched on top of the maps, with the lumen being easily identified by the lower modulus and hardness of the embedding resin. The modulus map (Figure 2.11(a)) shows that the elastic modulus in the wood cell wall varies from around $9 \mathrm{GPa}$ near the lumen and near the middle lamella to up to $32 \mathrm{GPa}$ in the middle of the cell wall. The hardness map (Figure 2.11(b)) shows a similar distribution, with values from $200 \mathrm{MPa}$ near the lumen and the middle lamella to values as high as $700 \mathrm{MPa}$ in the middle of the wood cell wall. Material variations and cell architecture can explain the mechanical response variations. The reduction in modulus and hardness near the lumen is presumably due to the decreased constraint by the lower stiffness and hardness of the embedding resin and low adhesion between the wood cell walls and the resin [129]. Reductions near the ML are presumably due to the high lignin content there. MFA also plays a role and is well known to affect the longitudinal stiffness: where the MFA is large, the stiffness is small and vice versus [130, 131]. However, the indents' size and spacings suggest that the known increase in MFA within $100 \mathrm{~nm}$ of the lumen and ML [12] will only produce secondary contributions to the measured decrease in modulus.

To minimize contributions from the lumen and the ML, indentation measurements were confined to the middle of the cell walls. To further identify the origins of measurement scatter, indents were compared that were placed in the middle of walls that are parallel to rays (radial walls), and in the middle of walls that are perpendicular to rays (tangential walls). The results shown in Figure 2.12 reveal that the standard deviations in both modulus and hardness are lowest for the tangential walls. The larger standard deviation of the radial walls may come from radial wall pits [17], which affect the orientation of the cellulose microfibrils in their vicinity. Therefore, all measurements to compare the effects of wood treatments are limited to indents positioned in the middle of the tangential wall cross-sections. We also avoided placing indents near rays. 


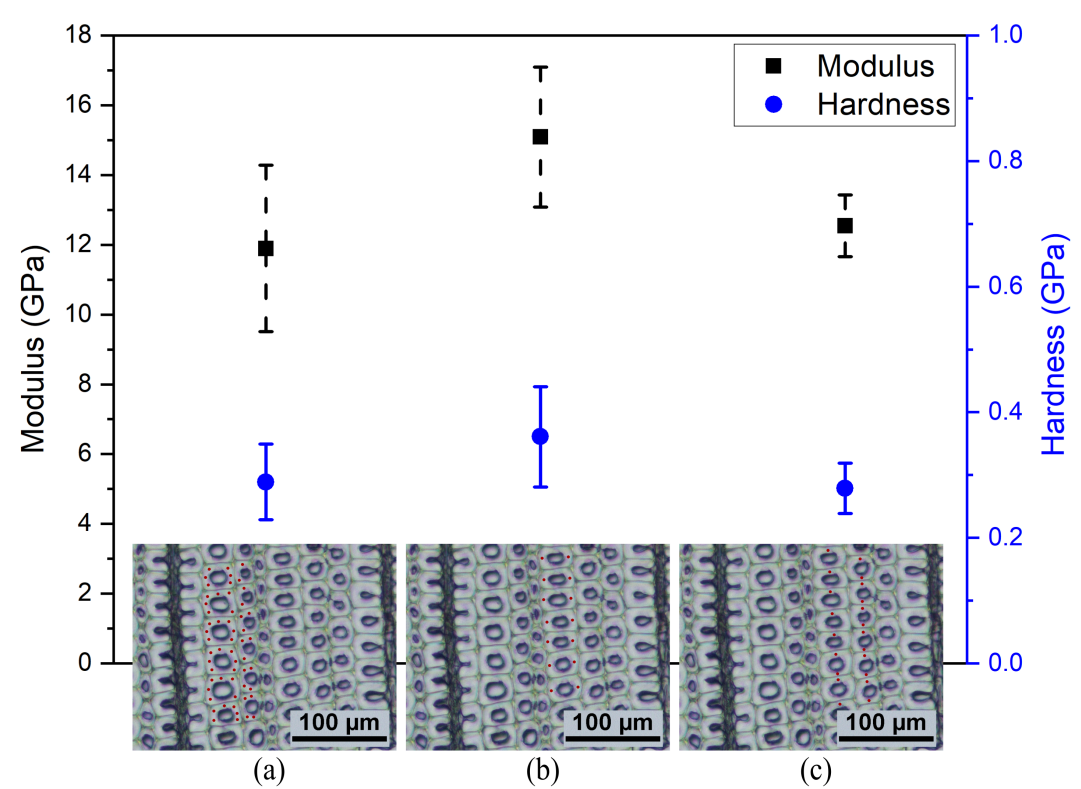

Figure 2.12: Modulus and hardness for pine wood axial cross-section obtained from (a) 30 indents placed randomly in the middle of the cell walls, (b) 10 indents placed in the middle of the radial walls, and (c) 20 indents placed in the middle of tangential walls. Error bars show the standard deviations. Modulus data are shown with square symbols and dashed line error bars. The Hardness data are shown with circle symbols and solid line error bars.

\subsection{Results}

Axial cross-sectional specimens of pine latewood and eucalyptus latewood were prepared for each of the three thermal treatments and indented both before and after treatment. The indents were performed in the middle of the tangential walls. Tests showing evidence of surface debris were culled out (e.g., Figure 2.8(b)), typically leaving between 10 and 30 high-quality indents for each treatment and wood type. The hardness, storage modulus, and loss modulus were calculated for each indent, as per the description in the previous section, and their means and standard deviations for each treatment and wood type are reported here.

The results for the pine and eucalyptus wood are summarized in Figure 2.13 and Figure 2.14. Hardness values for the untreated pine and eucalyptus latewood cell walls are around 0.5 GPa, and the storage modulus is around $13 \mathrm{GPa}$ for untreated pine latewood, 
WALL

and $15 \mathrm{GPa}$ for untreated eucalyptus latewood. The loss factors are around 0.10 for pine and 0.09 for eucalyptus wood, in agreement with the reported values of 0.06-0.09 of loss factor for dehydrated bulk bamboo samples in the frequency of $100 \mathrm{~Hz}$ [105].

\section{$\longrightarrow \triangle$ Before treatment}

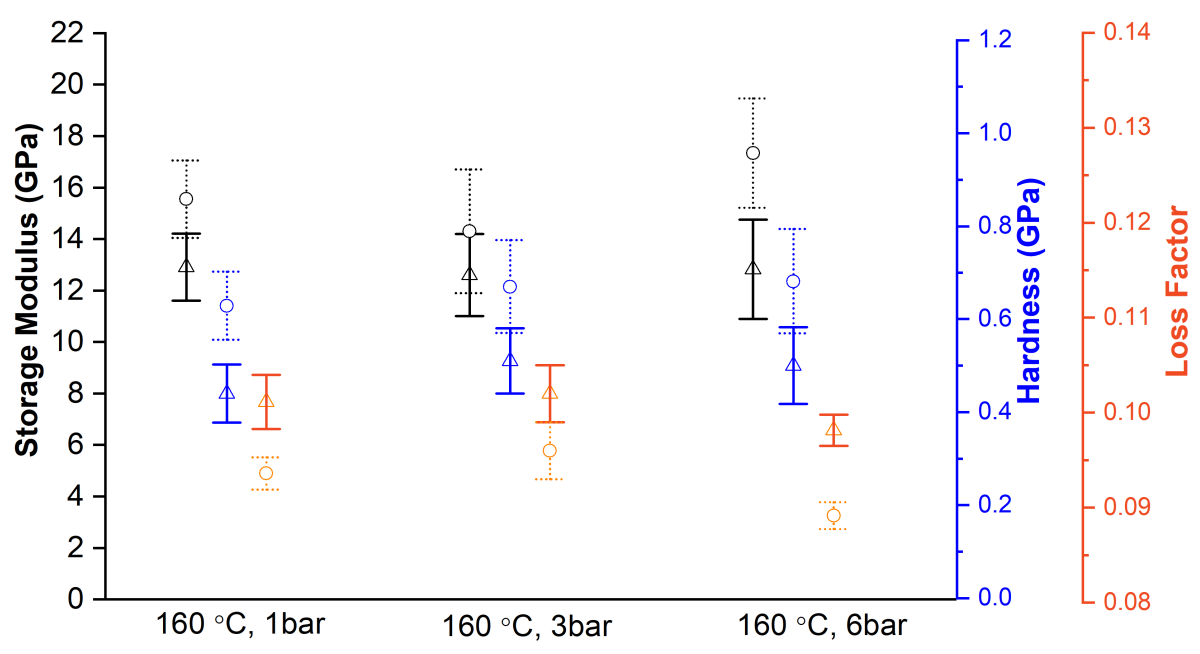

Figure 2.13: Storage modulus, hardness, and loss factor obtained from axial cross-sections of pine latewood before and after thermal modifications. Error bars show the standard deviations.

We note some variation in the means of the three untreated specimen populations for each wood type. In fact, the standard error, which is a measure of how far the mean of a given population may deviate from the true mean, is roughly a factor of 4 smaller than the standard deviation error bars, and is too small to explain the observed variation in means. This suggests that the variation in properties is smaller within the $1 \times 1 \mathrm{~mm}^{2}$ axial cross-sectional area of each specimen than between specimens that come from volumes that may have been up to $10 \mathrm{~mm}$ apart. This may either be an indication of a correlation length for property variations in wood, or it may reflect an apparent scatter due to deviations of the mounting angle of the wood specimens from the axial direction [73].

All three thermal treatments lead to an increase in storage modulus and hardness of the pine cell walls (Figure 2.13), whereas they have no significant effect on the storage modulus and hardness of the eucalyptus cell walls (Figure 2.14). In contrast, the loss factor clearly 
$\longrightarrow-$ Before treatment

......... After treatment

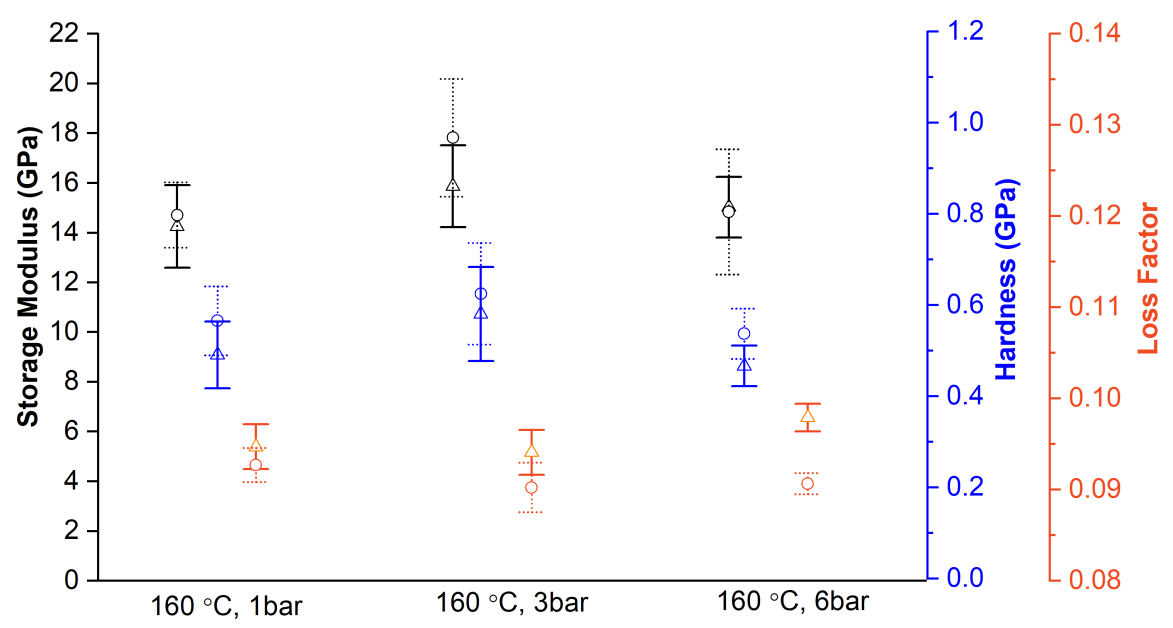

Figure 2.14: Storage modulus, hardness, and loss factor obtained from axial cross-sections of eucalyptus latewood before and after thermal modifications. Error bars show the standard deviations.

decreases for both types of wood as a result of thermal modification; the magnitude of the decrease appears to increase with humidity and pressure in the case of eucalyptus. The relative standard deviation in the loss factor is somewhat smaller than in the other properties, allowing changes due to thermal modification to be more easily seen.

Despite the large scatter in hardness and storage modulus, a comparison of the different quantities shows that they tend to scale with each other. This is illustrated by scatter plots of the hardness and the loss modulus versus the storage modulus for a given population of indents (Figure 2.15). Within the range of measured values, it is found that $\Delta H=$ $0.045 \Delta E^{\prime}, \Delta E^{\prime \prime}=0.080 \Delta E^{\prime}$, and $\Delta H=0.44 \Delta E^{\prime \prime}$. The strong positive correlation between the values clarifies that the scatter is not due to random effects but has a common origin. The obvious candidates for discussion are surface roughness and local variations in material properties. We note that the positive correlation between the loss and storage moduli also explains the smaller standard deviation of the loss factor shown in Figure 2.13 and Figure 2.14, since it is the ratio of the two moduli. 
WALL

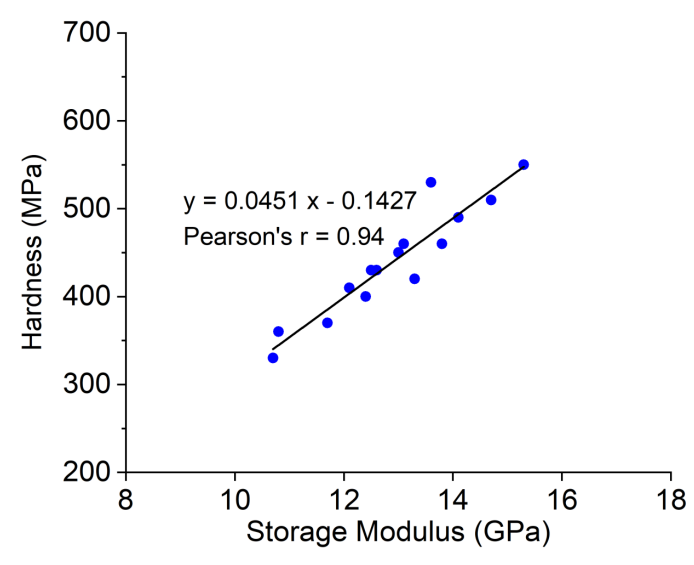

(a)

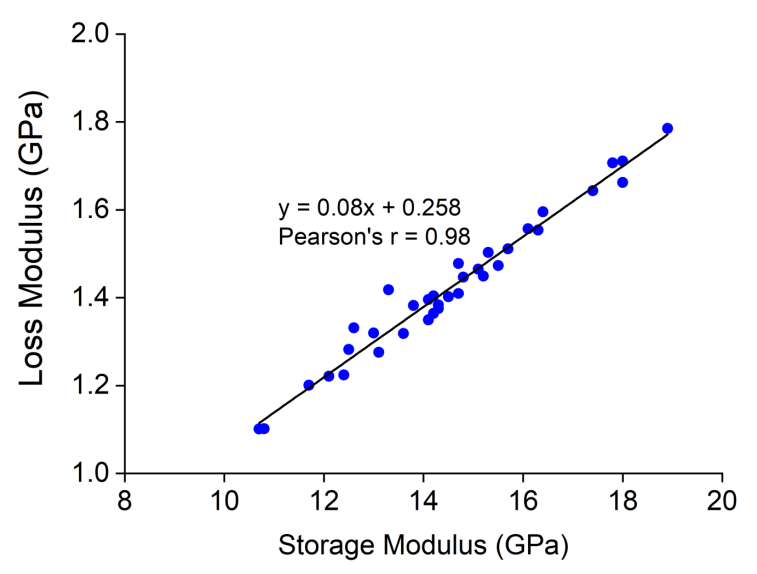

(b)

Figure 2.15: (a) Hardness vs. storage modulus and (b) loss modulus vs. storage modulus for the indents obtained from the untreated pine specimen.

The strong linear positive correlation between the loss and storage moduli is found to hold for all specimens in both the untreated and treated states (Figures 2.16 and 2.17). This offers a practical method to determine precise values for the loss factor, by simply fitting the data with a proportionality factor. The best proportionality fits before thermal treatment (green lines) and after thermal treatment (red lines) are included in Figures 2.16 and 2.17, and are summarized in Figure 2.18 and Figure 2.19. The results show a very clear decrease in the loss factor due to thermal treatments by around $10 \%$ in the pine latewood and a decrease as large as $8 \%$ in the eucalyptus latewood. Once again, we see that the error bars are smaller than the variations in the means. This strengthens the conclusion that the variations in properties (intrinsic or apparent) are smaller within the $1 \times 1 \mathrm{~mm}^{2}$ axial cross-sectional area of each specimen, than they are between specimens that come from volumes that are further apart.

\subsection{Discussion}

We have performed systematic measurements of the cell wall hardness, storage modulus, and loss modulus of pine and eucalyptus latewood using static and dynamic nanoindentation. The hardness and storage modulus values of the untreated cell walls fall within 


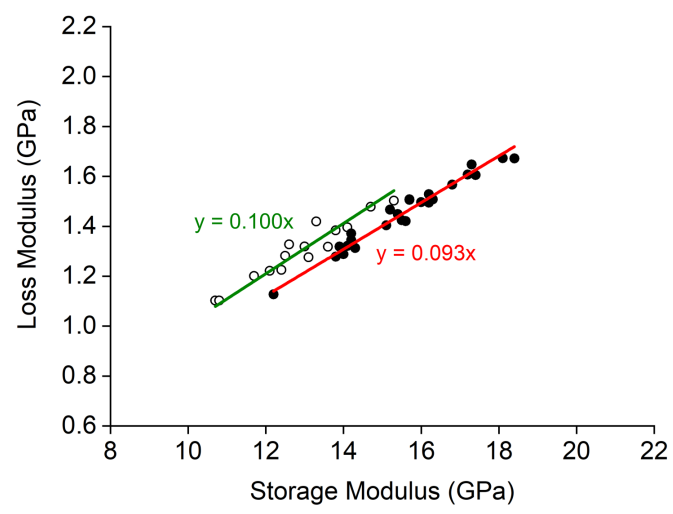

(a)

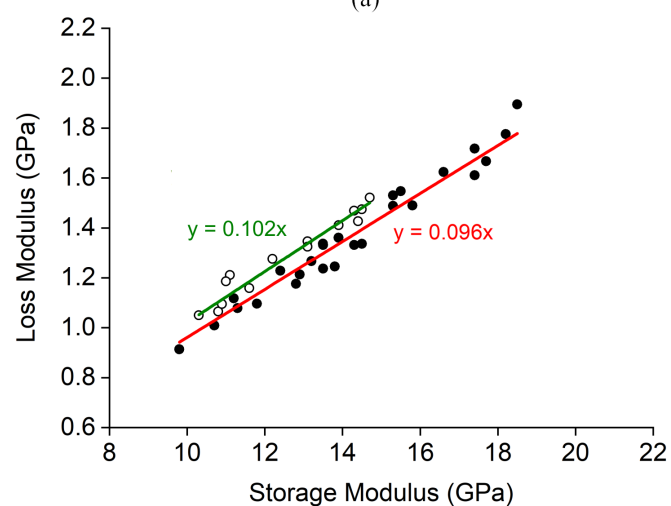

(b)

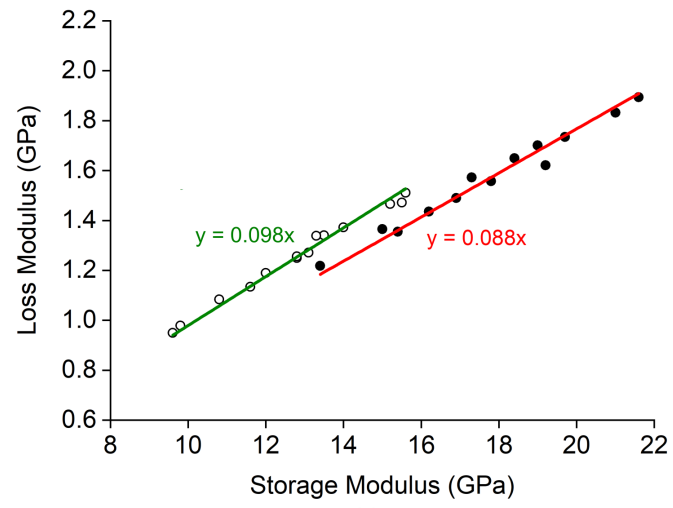

(c)

Figure 2.16: Loss modulus and storage modulus within the pine wood cell wall. Thermal modifications: (a) $160^{\circ} \mathrm{C}, 15 \% \mathrm{RH}, 1 \mathrm{bar}$, (b) $160^{\circ} \mathrm{C}, 50 \% \mathrm{RH}, 3 \mathrm{bar}$, (c) $160^{\circ} \mathrm{C}, 100 \% \mathrm{RH}$, 6bar. The green lines and open circles indicate the measurements of unmodified samples. The red lines and filled circles indicate the measurements of modified samples.

the range of previously measured values [72]. Consistent with previous microscale studies, thermal treatment leads to an increase in the hardness and storage modulus and a strong 


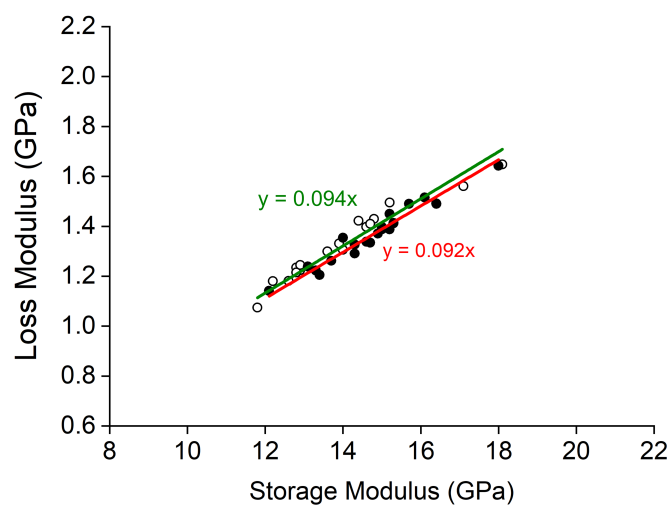

(a)

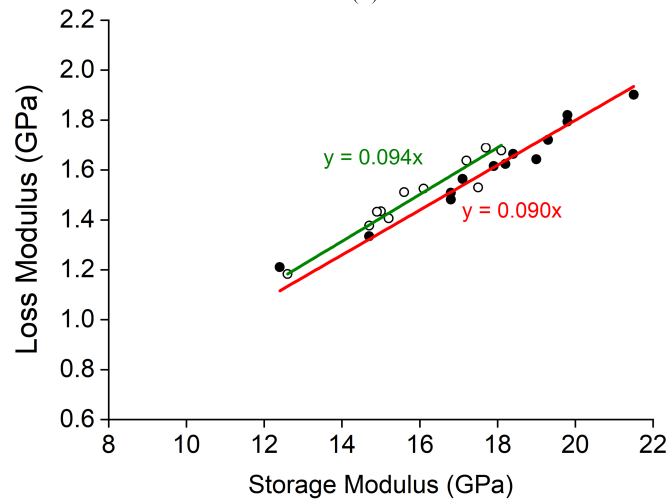

(b)

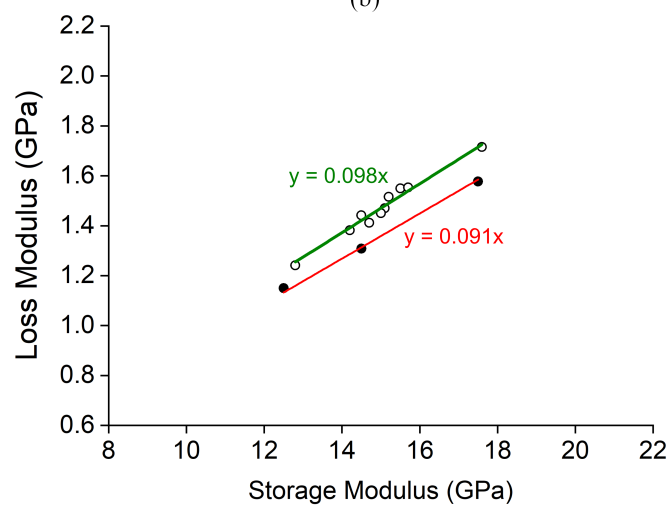

(c)

Figure 2.17: The correlation of loss modulus and storage modulus within the eucalyptus wood cell wall. Thermal modifications: (a) $160^{\circ} \mathrm{C}, 15 \% \mathrm{RH}, 1 \mathrm{bar}$, (b) $160^{\circ} \mathrm{C}, 50 \% \mathrm{RH}, 3 \mathrm{bar}$, (c) $160^{\circ} \mathrm{C}, 100 \% \mathrm{RH}, 6 \mathrm{bar}$. The green lines and open circles indicate the measurements of unmodified samples. The red lines and filled circles indicate the measurements of modified samples. 


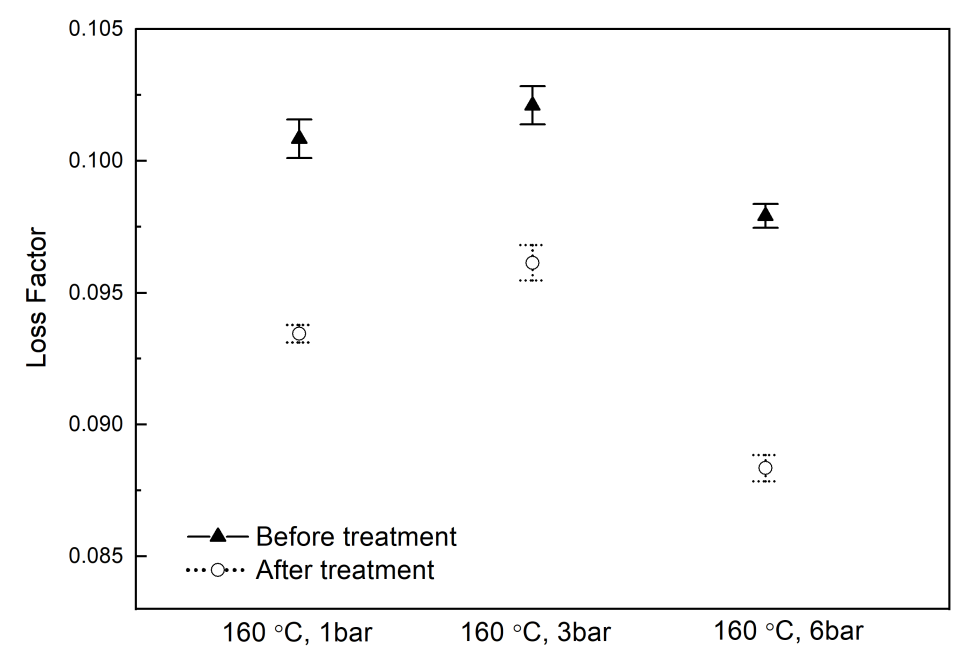

Figure 2.18: Loss factor for pine wood before and after thermal modification, as determined from the proportionality of the loss to storage modulus.

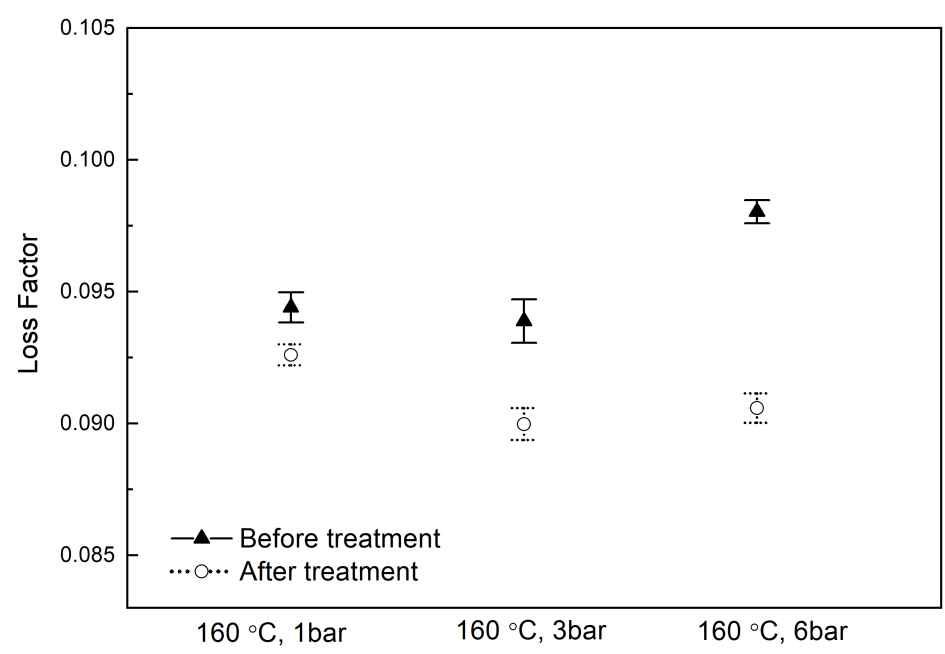

Figure 2.19: Loss factor for eucalyptus wood before and after thermal modification, as determined from the proportionality of the loss to storage modulus. 
WALL

decrease in the loss factor $[118,132,133,134]$. Despite the care taken in positioning the indents in specific locations in the cell wall, a large scatter of up to $40 \%$ was observed in the local mechanical response. The scatter in all measured properties showed strong positive correlations, revealing that they have a common, deterministic origin. Possible origins for these correlations and for the effects of thermal modification on the mechanical properties of the cell walls are discussed below and compared with macroscale mechanical properties of thermally treated wood. But first, we start by discussing the state-of-the-art understanding of the effect of composition and architecture on mechanical properties within the context of the fiber-reinforced composite theory.

\subsubsection{Composite model for the wood cell wall}

The mechanical properties of wood cell walls have often been modeled as cellulose fibers embedded in a matrix composed of lignin and hemicellulose, using fiber-reinforced composite theory. The wood cell wall mechanical properties will depend on the cellulose volume fraction $(f)$ and on the angle of the microfibrils relative to the loading axis. For the case of axial loading, this angle can be approximated as the MFA in the tested volume.

It is generally found that constant strain $\left(E=(1-f) E_{L}+f E_{C}\right)$ and constant stress $(1 / E=$ $\left.((1-f)) / E_{L}+f / E_{C}\right)$ models give reasonable agreement with the fiber volume fraction dependence of the Youngs modulus for loading angles of $0^{\circ}$ and $90^{\circ}$, respectively [135]. These models are shown in Figure 2.20(a) for cellulose fiber-lignin matrix composites, where the elastic modulus of cellulose and lignin are estimated to be $138 \mathrm{GPa}$ and $3.1 \mathrm{GPa}$, respectively $[21,136]$. Moreover, the volume fractions of cellulose in pine and eucalyptus are defined as 0.5 and 0.55 accordingly.

Although the predicted values are defined for MFA of $0^{\circ}$ and $90^{\circ}$, the nanoindentation measurements are performed mostly on the S2 layer with MFA between $5^{\circ}$ and $20^{\circ}$. Therefore, the differences between the calculated values and the nanoindentation values are explainable. Furthermore, the nanoindentation elastic modulus of the wood cell wall reflects a combination of both axial and transverse properties, which causes an underestimation of the higher modulus. 


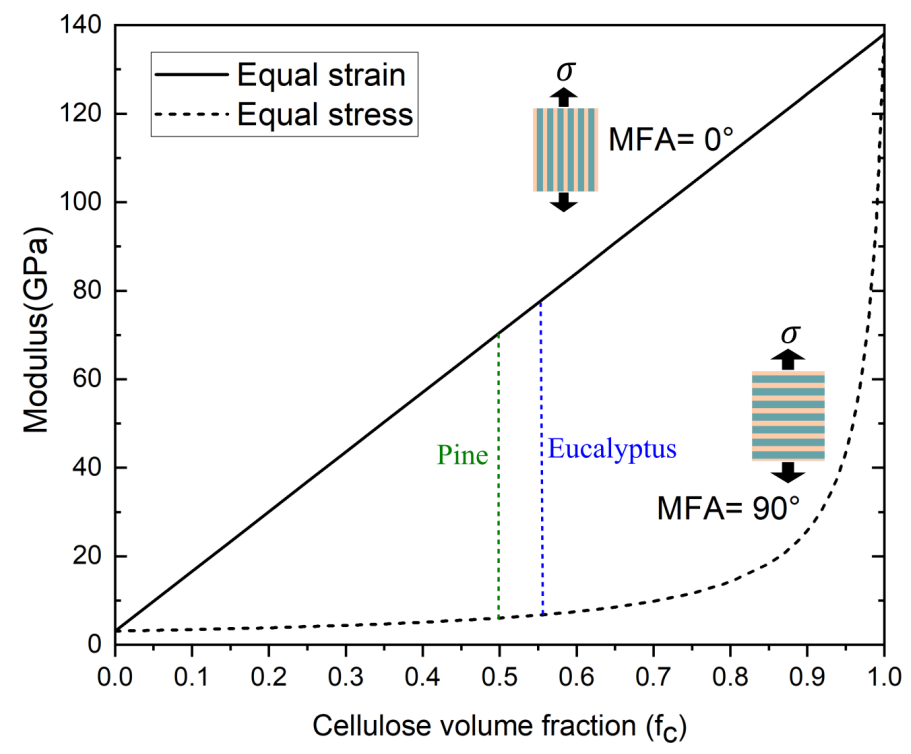

(a)

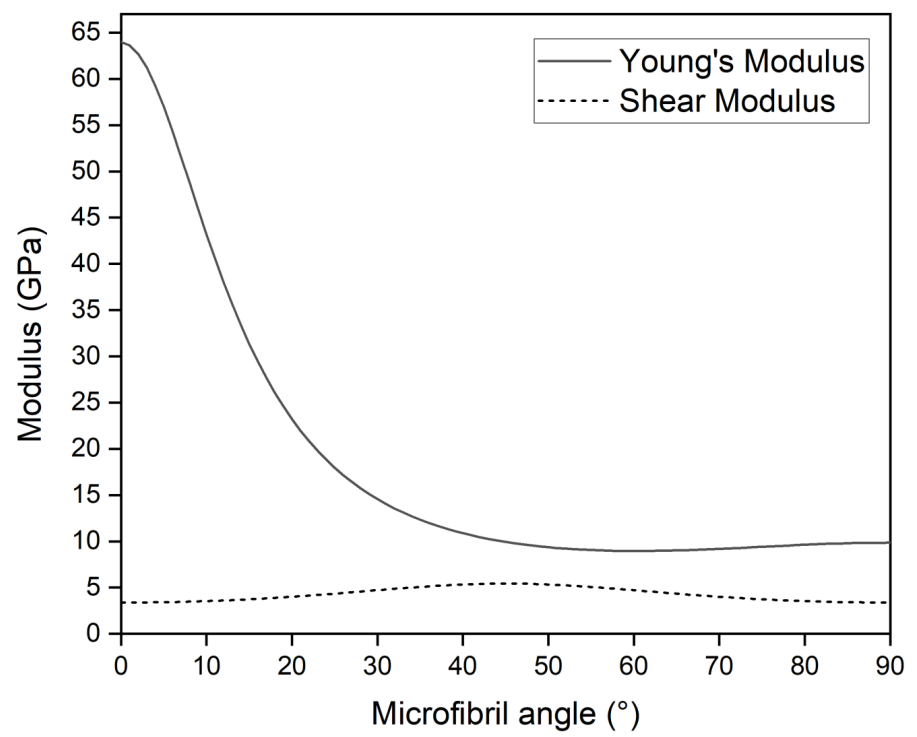

(b)

Figure 2.20: (a) The corresponding prediction of the wood cell wall's storage modulus according to the cellulose volume fraction fc by using the Rule of Mixtures in fiber-composite theory. (b) Variation of Young's modulus and shear modulus in pine wood as a function of MFA according to the laminar composite theory [135, 137]. 

WALL

The dependence of Young's modulus and shear modulus on the loading angle is shown in Figure 2.20(b). The graph is plotted based on the model for a laminar composite structure [135] and according to the elastic constants in the S2 layer given by Harrington et al. [137] for Pinus radiata pine at a $12 \%$ moisture content (Table 2.3).

Table 2.3: The constituents mass fraction and elastic constants (in GPa) of the S2 layer [138].

\begin{tabular}{lccccccc}
\hline \hline Wall layer & $f_{C}$ & $f_{H}$ & $f_{L}$ & $E_{t}$ & $E_{l}$ & $v_{l t}$ & $G_{l t}$ \\
\hline S2 & 50.4 & 27.8 & 22.0 & 9.85 & 63.96 & 0.33 & 3.38 \\
\hline \hline
\end{tabular}

Figure 2.21 shows the variation of longitudinal Young's Modulus with mean microfibril angle in pine wood [139]. In agreement with the laminar composite theory, the experimental data also show that longitudinal Young's modulus reduces steeply as the MFA in the cell wall increases.

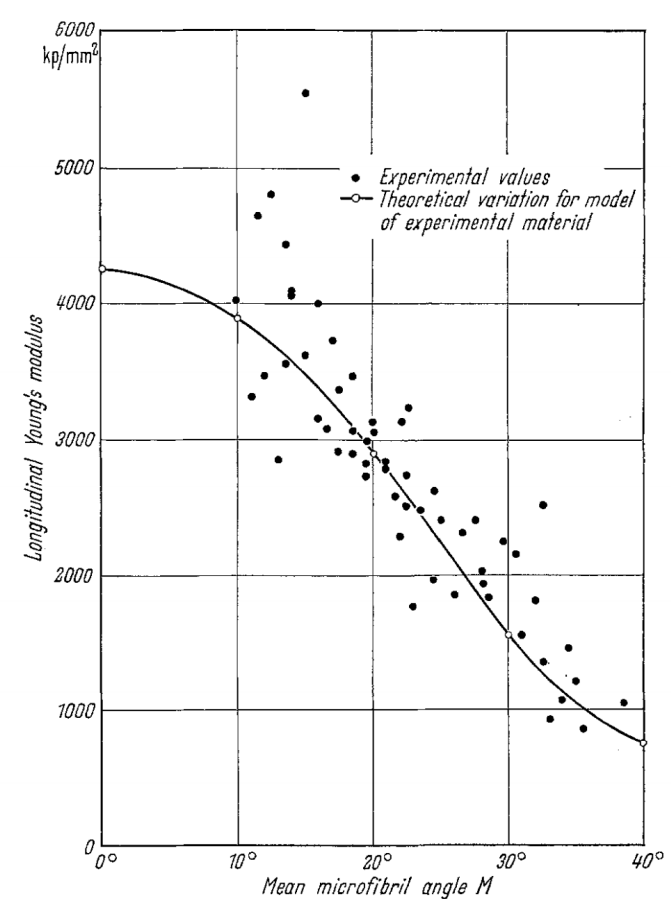

Figure 2.21: The longitudinal Young's modulus of Pinus radiata according to the MFA. A Comparison between the proposed model shown by the solid line, and experimental data in black dots. Reprinted by permission from Springer Nature [139], Copyright 1968. 
Composite theories for strength are complex and have not been applied as extensively as theories for elasticity to explain the behavior of wood cell walls. It is nonetheless widely assumed that the strength, whether measured under tension or compression, is controlled by the cellulose fibers, which have much higher strengths than the lignin [20]. A study of nanoindentation hardness, which is directly proportional to strength, supports the idea that cellulose has the dominant effect [92].

A number of models have been developed for the compressive strength of composites depending on their deformation or failure mode, which in turn depends on the misalignment between the fiber direction and loading direction. Following the model of independent modes of failure [135] and using experimental data of axial strength $\left(\sigma_{1 u}=100 \mathrm{MPa}\right.$ [141]), transverse strength $\left(\sigma_{2 u}=20 \mathrm{MPa}[141]\right)$, and shear stress $\left(\tau_{12 u}=6.6 \mathrm{MPa}[142]\right)$, the maximum strength of the S2 layer with fibers oriented at a given MFA is plotted in Figure 2.22.

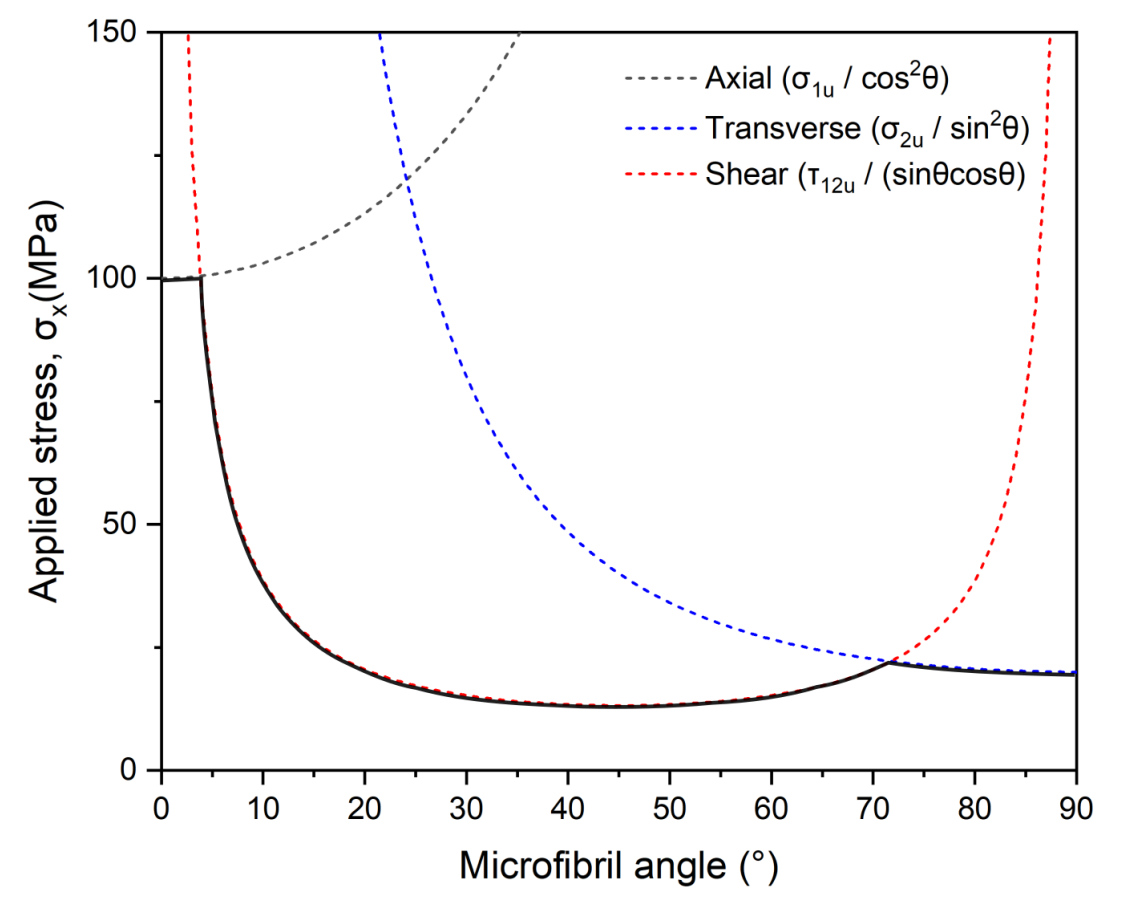

Figure 2.22: The prediction of different failure modes' onset regarding the angle between applied stress and fibrillar arrangement. Following Hull and Clyne [135].

For the case of nanoindentation into S2 layers, the angle of the Berkovich pyramid faces 
WALL

( $25^{\circ}$ between face normal and indent direction) and the MFA of the S2 cellulose fibers (typically between $5^{\circ}$ and $20^{\circ}$ ) leads to misalignment between the fiber direction and the surface-near loading direction between $5^{\circ}$ and $45^{\circ}$. According to models for the compressive strength of engineering long fiber and laminate composites [135], deformation during nanoindentation testing is expected to occur in wood cell walls by kink band formation. Regarding (Figure 2.22), the local failure mode for the S2 layer loaded in the longitudinal direction (by MFA of $5^{\circ}$ and $45^{\circ}$ ) could be failure parallel to the microfibrils or shear along the fibrils. Both failure modes show a decrease in failure stress with increasing misalignment. Thus, nanoindentation is expected to give values for the hardness and strength, which are up to ca. $80 \%$ smaller than the values for pure axial loading, as a result of the wood MFA and the tilting of the indenter tip pyramid faces.

As for the case of stiffness, the strength and hardness also depend on the cellulose to lignin fraction, such that the more cellulose, the higher the hardness. For example, in the case of kink band formation, the rate of decrease of strength/hardness with misalignment angle scales with the composite shear modulus $G$, which is given by the constant stress model $1 / G=(1-f) / G_{L}+f / G_{C}$. In contrast, the failure stress for splitting likely does not depend strongly on the cellulose fraction.

Damping, viscoelastic behavior, and loss factor of wood cell walls have only been reported in a few publications [83, 92, 104]. The study by Wang [92] identifies hemicellulose as the dominant contributor to viscous behavior. This seems reasonable given the velcro model of Fratzl et al. [65]. A possible simple model for this behavior is then based on the idea that dissipation happens by shear motion in the hemicellulose layers that bond the cellulose to lignin. Consistent with Adams et al. [143], who mentioned that the friction of fibers against the matrix and the shearing stresses affected by hydration and chemical forces are the main factors in dissipation energy in the composite materials. The viscoelastic behavior of the interface between polymeric components produces more dissipation than the viscoelastic behavior of each polymer itself. Loss modulus should then be proportional to the interface area per unit volume and the resolved interface shear stress, which depends on misalignment so that $E^{\prime \prime}=E_{0}^{\prime \prime} \frac{2 \pi r}{a^{2}} \sin \theta \cos \theta$, where $f=\pi(r / a)^{2}$, so that $E^{\prime \prime}=E_{0}^{\prime \prime} \frac{2 f}{r} \sin \theta \cos \theta$. Here $r$ is the constant microfibril radius and a is the distance between fibrils. $r$ is fairly constant and assume $E_{0}^{\prime \prime}$ is as well (loss per unit area at small misalignment), so that $E^{\prime \prime}$ is expected to scale linearly with $f$ and to increase with misalignment in the range below $45^{\circ}$. We note 
that while $E^{\prime}$ also increases with $f$, it will decrease with misalignment, so that local MFA cannot explain the positive correlation between $E^{\prime}$ and $E^{\prime \prime}$.

In Appendix A, the correlation of $E^{\prime}$ and $E^{\prime \prime}$ has been considered with a three-phase composite model for cell wall including interfaces as the third phase in the laminar structure, which is in agreement with the conclusion that loss modulus will increase with increasing the volume fraction of the cellulose fibers, up until the point that the cellulose fibers touch.

\subsubsection{Origin of scattering in local mechanical properties}

Local variations in the mechanical response of wood have long been known and attributed to variations in the component compositions and to the angle of the cellulose microfibrils (MFA) relative to the loading axis $[69,70,73,144]$. We re-discuss these effects here, in light of our new observation that the scatter in the different properties is strongly correlated, and additionally discuss the effect of surface roughness on the calculated values of the mechanical properties.

Based on the definitions of hardness, storage modulus, and loss modulus (Eq. 2.6a), it is clear that both local material properties and true contact area determine their values. The true contact area may differ from the calibrated value due to sink-in and pile-up (which are determined by local mechanical properties), but it is also affected by surface roughness. The variations in surface height as large as $\pm 10 \mathrm{~nm}$ in the axial cross-sections of the cell walls lead to relative uncertainties in the displacement into a surface as large as $\Delta h / h=10 \%$ at a depth of $100 \mathrm{~nm}$, where the mechanical properties are recorded. Given that the projected contact area of a spherical body of radius $R$ displaced into a flat surface is $A=\pi R h$, the relative uncertainty in the contact area $\Delta A / A$ at a depth of $h=100 \mathrm{~nm}$ is also $10 \%$. It follows immediately from Eq. 2.6a that the relative uncertainties in hardness and moduli due to surface roughness are $10 \%$ and $5 \%$, respectively.

The observed standard deviation in hardness and moduli values are approximately $40 \%$ and 20\%, respectively (Figure 2.13 and Figure 2.14). Although these standard deviations have the expected ratio for variations caused by uncertainties in the true contact area, they are larger than predicted by the measured roughness. Given that the roughness occurs at 

WALL

length scales smaller than the tip radius (Figure 2.2), the RMS roughness of $40 \mathrm{~nm}$ would be necessary to explain the results. This can be ruled out, leaving us with the conclusion that surface roughness can only partially account for the observed scatter. We note again that the precision of the surface find routine is better than $1 \mathrm{~nm}$, so that it also can not account for the observed scatter.

Variations in local material properties that occur on a length scale larger or equal to the indentation interaction volumes will cause variations in measured local mechanical properties. For the tests performed here, we expect plastic interaction volumes with dimensions of 1 to $2 \mu \mathrm{m}$, while the elastic interaction volumes are larger. Deviations in the true contact area will lead to positive correlations, as observed here, due to the fact that all properties increase with decreasing contact area. Similarly, a positive correlation is expected between hardness and storage modulus, since hardness measures a material response determined by both elastic and plastic contributions. Hardness has been observed to increase linearly with elastic modulus [145]. So that the positive correlation between hardness and storage modulus does not reveal anything new. However, the positive correlation between loss modulus and storage modulus is more intriguing, and exploring the conditions under which this can occur may give us insights into the origins of the structural features that control loss and storage moduli.

The loss modulus results from the viscous properties of polymeric-based materials and represents energy lost during cyclic loading. The primary source of dissipation in fiberreinforced composites is believed to occur due to friction at the interface of the cellulose fibrils to the surrounding material [143]. Given that hemicellulose acts as a bonding layer between the cellulose and lignin, this is consistent with a study showing that hemicellulose is the main source of viscoelastic dissipation in the cell wall [92]. A simple model based on these ideas would predict that the loss modulus should increase with the interface area, and therefore with the cellulose fraction, and with MFA up to $45^{\circ}$. This dependence on MFA is supported by the study by Roszyk et al. [146]. In contrast, studies on the storage modulus of the cell walls show a decrease with MFA (Figure 2.21 and Figure ??). This suggests that the scatter observed here is not the result of MFA variations. This is somewhat surprising since many studies show pervasive variations in MFA at a variety of different length scales [147]. However, local variations in composition remain a possible explanation for the scatter since an increase in cellulose content is predicted to cause an increase in both 
the storage and loss modulus. We note that changes in the local cellulose volume fraction up to $50 \%$ would be needed to explain the observed scatter. Thus, we propose that the strongly correlated scatter in the micromechanical properties measured in the cell walls of pine and eucalyptus is due to a combination of surface roughness and local variations in cellulose fraction $\left(E^{\prime \prime}=E_{0}^{\prime \prime} \frac{2 f}{r} \sin \theta \cos \theta\right)$.

The correlation between hardness and modulus is reported in the other studies as well [93, 145, 148, 149]. Labonte et al [145]. reported that for a wide range of biomaterials, the indentation hardness was tended to be linearly related to the indentation modulus, with a constant proportionality of about $0.05\left(H=0.046 E^{1.03}\right)$. This ratio was addressed to the amount of relative irreversible deformation in the contact zone, the material's yield strength, and the severity of mechanical damage.

Indentation hardness is a hybrid property that measures both reversible and irreversible deformation resistance and therefore is intrinsically related to the elastic modulus. Furthermore, it has been shown that hardness is a measure of the work needed to produce a unit volume of residual impression [150]. So, as a result, the ratio of hardness to modulus is related to the ratio between irreversible and reversible work performed throughout indentation [151]. This ratio, which reflects the energy needed to produce a unit volume of irreversible impression, is independent of the modulus and indenter geometry [145].

\subsubsection{Origin of the effect of thermal modification on micromechan- ical behavior}

The thermal treatments lead to mass loss, degradation of hemicelluloses, cross-linking of lignin, and an increase in the crystalline fraction of cellulose. At the low treatment temperature $\left(160^{\circ} \mathrm{C}\right)$ used in the current study, basically, only the loss of amorphous carbohydrates included amorphous cellulose and hemicelluloses, can be expected. Therefore, the observed decrease in hydrophilicity (Figure 2.3) is expected by losing the hemicelluloses after thermal treatment.

The mass reduction of wood is due to thermal modification is presented in Figure 2.4. According to the literature, the main reasons for the mass loss are degradation of wood 

WALL

components (mainly hemicelluloses) into volatile products that evaporate during treatment, evaporation of extractives, and loss of moisture content. The main forms of degradation caused by thermal treatment are cleavage of secondary bonds included hydrogen and Van der Waals bonds, within the hemicellulose molecules, cleavage of the secondary bonds between the hemicellulose and cellulose, cleavage of covalent bonds between hemicellulose and lignin, and cleavage of covalent bonds within the cellulose fibrils (depolymerization) [33].

Heat treatment under moist conditions, especially in the treatment of $100 \% \mathrm{RH}$, appears to increase the fraction of crystalline cellulose [37, 38, 41, 42, 152]. Bhuiyan et al. [38] mentioned that crystal cellulose fraction after a highly moist thermal modification is almost two times higher than crystal cellulose fraction after oven-dried thermal modification. They suggested that the crystallization of cellulose under the highly moist heat treatment condition enables the hydrogen bonds to make the system more flexible for adopting and reordering the crystalline chains. Moreover, the internal stress could distribute among the molecular chains more uniformly and prevent severe shrinkage in the cell wall under the hydrothermal modification.

The researchers are wonder if the increased crystal cellulose fraction is due to the degradation of amorphous cellulose rather than the crystallization of amorphous cellulose. Boonstra et al. [33] indicated that at temperatures below $210-220^{\circ} \mathrm{C}$, only a little cellulose degradation occurs; therefore, the degradation of cellulose seems to be unlikely in the thermal modifications of the current study.

As seen in Figure 2.13, there is an increase in the elastic modulus of pinewood after thermal modification. Consistent with other nanoindentation studies [118, 132, 133, 134]. Easy to understand in terms of crystallization of cellulose which is stiffer than amorphous cellulose. As shown in Figure 2.14, the elastic modulus of the eucalyptus wood cell walls seems to have not significantly changed after thermal modification. One possible explanation could refer to the crystalline forms of cellulose in softwoods and hardwoods. The relative amount of cellulose $\mathrm{I} \beta$ and cellulose $\mathrm{I} \alpha$ is different in different wood spices. According to the research of Roger $H$. Newman [153], the ratio of I $\alpha$ to I $\beta$ crystalline forms of cellulose are noticeably higher for softwoods than for hardwoods. It is reported in different works of literature that cellulose I $\alpha$ could be transformed into I $\beta$ by hydrothermal treatment [154] or by heat treatment in inert gas [24]. This demonstrates that cellulose $\mathrm{I} \beta$ is thermodynamically 
more stable than cellulose I $\alpha$ [21]. It means the cellulose I $\beta$ form in eucalyptus wood (as hardwood) is more stable under thermal modification, but the cellulose $\mathrm{I} \alpha$ form is metastable and can be converted to the more stable I $\beta$ in pine wood (as softwood) after thermal modification.

According to the hardness measurements in pine wood samples (Figure 2.13), there is an increase in hardness after thermal modification. During the hydrothermal treatment, lignin could be affected by degradation and also depolymerization reactions. The covalent bonds between lignin and hemicelluloses might be broken. Consequently, the high reactive products of low molecular weight lignin fragments and the degradation products of hemicelluloses could produce new lignin-based polymers and increase cross-linking of the existing lignin network [40].

Although the resistance to plastic deformation is related to covalent bonds but also hydrogen intrapolymer bonds could play an important role. Dumail et al. [155] suggested that the breakage and reformation of hydrogen bonds have a crucial role in the stress-strain behavior of wood. It is widely accepted that the strength properties of wood are affected by bound water. Decrease amounts of bound water increase hydrogen bonding between the organic polymers of the cell wall and thereby increase the strength properties of wood. Heat treatment, therefore, has a positive contribution to the strength properties since heattreated wood is less hydrophobic. As shown in Figure 2.3, the moisture content is reduced after thermal modification. The lowest moister content for the treatment of 1 bar pressure and $15 \% \mathrm{RH}$ correlated well to the higher increase of hardness in the wood cell wall for this treatment.

The loss factor of the wood cell walls was significantly reduced by thermal modification (Figure 2.13 and Figure 2.14). As discussed previously, the main source of dissipation is believed to be frictional sliding between cellulose fibers and the surrounding matrix. However, following the research on damping in fiber-reinforced composite materials [156, 157], we expect that the viscoelastic behavior of the cellulose and lignin components will also play a role. Further, hydroxyl groups and moisture content, hemicellulose, and any damage or defects are expected to influence damping.

As shown in Figure 2.23 and regarding the idea of Roger Rowell. [20], when a load is 
WALL

applied to a piece of wood, at the molecular level, hydrogen bonds within the individual cellulose chains are breaking and subsequently reforming to allow the elementary fibrils to slide by one another [20]. This load distribution within the microfibrils contributes to the distribution of internal stresses and, consequently, energy dissipation in the whole system [158].

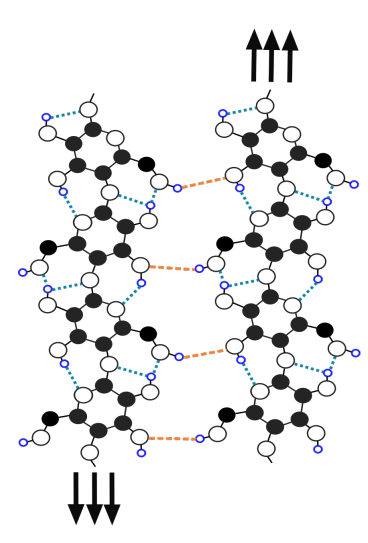

(a)

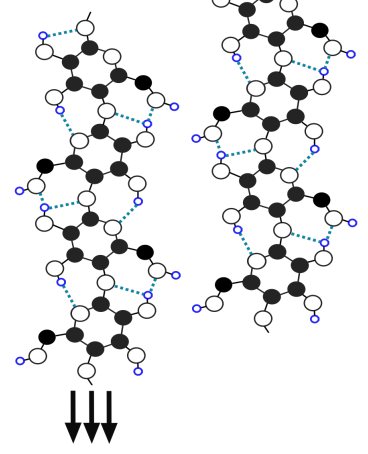

(b)

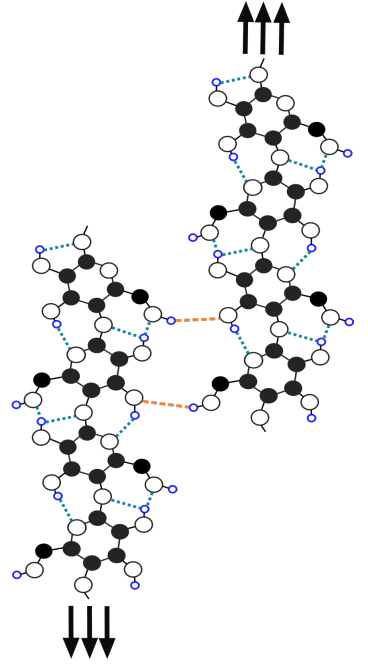

(c)

Figure 2.23: Hydrogen-bonding patterns in cellulose I $\beta$ [88] under shear forces. (a) bonded (b) sliding, unbounded (c) rebounded [20]. Black circles: Carbon atoms, White circles: Oxygen atoms, Blue circles: Hydrogen atoms, Orange dashed lines: inter-chain hydrogen bonding, Blue dashed lines: intra-chain hydrogen bonding. Adopted with permission from J. E. Winandy et al. [20], Copyright 1984, American Chemical Society.

Furthermore, in the wood cell wall, interphases between the cellulose fibers and the matrix of hemicelluloses and lignin are assembled with the chemical-mechanical linkages originated from covalent and hydrogen bonds [20, 158]. It has been suggested from different works of literature that the primary function of hemicelluloses is to act as a coupling agent between hydrophilic cellulose and hydrophobic lignin. The carbohydrate molecules of hemicelluloses are highly branched and expose hydrogen bonding within the hemicellulose chain and between hemicellulose and cellulose regions [20]. Since hemicelluloses are the most sensitive component of wood to thermal and chemical modifications, the high temperature and high relative humidity during the hydrothermal treatment resulted in a significant reduction of hemicellulose (Figure 2.5). 
Hemicellulose presumably acts as an essential factor for dissipating energy in the wood cell wall. Degradation of the hemicelluloses interrupts the load-sharing capacity of the ligninhemicellulose matrix in which the cellulose fibrils are embedded. Therefore, the ability of cellulose fibrils to distribute the stress in the system will be reduced [20, 33, 116, 159]. (see Figure 2.24)

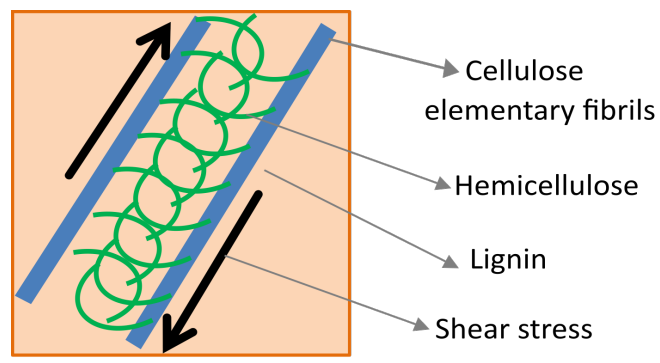

(a) Unmodified wood

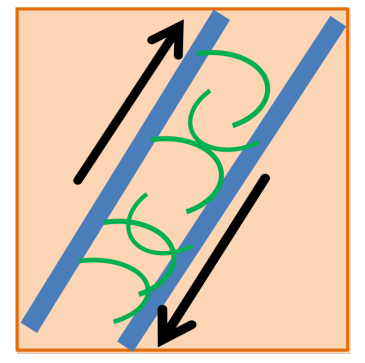

(b) Thermally modified wood

Figure 2.24: Interfacial slippage between elementary fibrils with hemicellulose contribution: (a) before thermal modification, (b) after thermal modification.

Another important factor to consider is the availability and accessibility of the free hydroxyl groups of the wood carbohydrates [33] to the adsorption and desorption of water molecules in the wood cell wall. As shown in Figure 2.3, there is a significant reduction in moisture content after thermal modification. The reduction of free hydroxyl groups could be related to depolymerization of the carbohydrates and especially hemicelluloses and increase of the relative proportion of the crystalline cellulose, in which the hydroxyl groups are not easily accessible to water molecules [32, 160], and cross-linking of the lignin network [32] which might prevent the accessibility of free hydroxyl groups to water [161]. This reduction of moisture content after thermal modification presumably is one of the reasons for the lower damping factor after thermal modification [162].

The thermoplastic behavior of polymer chains is also one of the facts that should be discussed concerning thermal modification. It is mentioned in the works of literature that the hemicellulose could pass the rubbery or plastic state within the $127-235^{\circ} \mathrm{C}$. For lignin, the transition state is in the range of $167-217^{\circ} \mathrm{C}$, and for cellulose, it is much higher and is around $231-253^{\circ} \mathrm{C}$. However, by counting the role of water as a plasticizer, steaming could reduce the softening point of each component by about $20^{\circ} \mathrm{C}$ [33]. So it seems that in the current thermal modification that we used, the temperature of $160^{\circ} \mathrm{C}$ might enable 
WALL

the hemicellulose and lignin to reach the rubbery state. After the cooling down phase, the polymer chains become rigid again. This kind of phase transformation could change the molecular structure and affect the damping behavior as well [33].

\subsubsection{Comparison of micromechanical behavior with macroscale properties}

The macro mechanical measurement of thermally modified wood samples in bulk scale has been provided in different research works and depends sensitively on the details of the thermal treatment (temperature, whether closed or open, whether dry or wet). Wentzel et al. reported that thermal modification of eucalyptus wood samples in the closed system under the condition of $160^{\circ} \mathrm{C}$ temperature and $100 \% \mathrm{RH}$ caused a decrease in the modulus of elasticity (MOE) from 18.45 $\pm 0.96 \mathrm{GPa}$ to $15.06 \pm 1.7 \mathrm{GPa}$, whereas the thermal modification of $160^{\circ} \mathrm{C}$ temperature and $30 \% \mathrm{RH}$ had a slight decrease of MOE from $18.45 \pm 0.96$ $\mathrm{GPa}$ to $17.01 \pm 1.2 \mathrm{GPa}$ [45]. It means that the changes in the bulk elastic properties of eucalyptus wood under lower pressure treatment followed the same trend as the microscale behavior of the eucalyptus treated samples. On the other hand, the higher pressure treatment induced a decrease in bulk elastic properties while having no effect on the micro elastic properties of eucalyptus wood (see Figure 2.14).

Michiel J. Boonstra et al. [33] mentioned that the Brinell hardness of pinewood samples in the axial direction increased by about $48 \%$ after hydro thermolysis treatment under relatively mild conditions $\left(<200^{\circ} \mathrm{C}\right)$, which is consistent with the increased hardness observed in the indentation test of thermally treated pine wood samples in this study (see Figure 2.13).

Furthermore, Boonstra et al. [33] reported that the tensile strength parallels to the fibers decreased $(39 \%)$. In contrast, the compressive strength parallels to the fibers increased $(29 \%)$ after heat treatment. This treatment also resulted in a small reduction (3\%) of the specimen's strength before rupture (MOR).

Wentzel et al. [113] reported a decrease of MOR at the modifications with higher pressure $\left(160^{\circ} \mathrm{C}, 6 \mathrm{bar}\right)$ from $119 \pm 13 \mathrm{MPa}$ (untreated) to $89 \pm 15 \mathrm{MPa}$ (treated). However, the 
modifications at a lower pressure $\left(160^{\circ} \mathrm{C}, 1.8\right.$ bar $)$ did not have a significant change in MOR $(117 \pm 16)[94]$. It is mentioned in their research that thermal modification on the eucalyptus wood samples initiated the cracks in the middle lamella and pits.

So far, the dynamic mechanical analyses and loss factor measurements of wood in macroscale have been barely employed. However, there are several studies related to dynamic strength testing, such as the impact bending test in the bulk scale. The damping and impact strength are both a function of temperature. In the research of Heijboer et al. [163], it is mentioned that if the damping is because of the movement within the main polymer chains, the damping and compact strength correlate with each other, and the maximum damping causes an increase in impact strength. Despite that, if movements of sidechains only cause the damping maximum, it has a slight influence on the impact strength.

The impact strength is defined by the lost energy of the hammer through the breakage of the specimen. This energy is the sum of three quantities: the energy required to fracture the specimen, the energy to throw the broken end of the specimen, and the energy dissipated in the device supporting the fixed end of the specimen. The information provided by Kubojima et al. [164] and Boonstra et al. [33] showed that the decrease in dynamic strength after thermal modification is more drastic compared to MOE and MOR. This agrees with damping measurements in the current study, where a clear decrease in loss factor with thermal treatment is observed for both pine and eucalyptus wood.

Furthermore, a new test method named the High-Energy Multiple Impact (HEMI) test was developed by Rapp et al. [165] to overcome the difficulties of standard dynamic strength testing. This method is based on crushing small specimens by a great range of impacts from pounding steel balls in a heavy vibratory mill. Wentzel et al. [113] measured the dynamic mechanical behavior of eucalypt wood after thermal modification using a HighEnergy Multiple Impact (HEMI) method. They calculated resistance to impact milling (RIM) and the degree of integrity (the percentage of the biggest fragments) of modified and unmodified wood samples. An obvious reduction in RIM and degree of integrity was observed for thermally modified eucalyptus wood samples. Rapp et al. [165] indicated that RIM reduction is highly correlated with the decrease in mass due to heat treatments.

In general, it seems that under the mild thermal treatment conditions, the bulk and mi- 

WALL

croscale mechanical behavior of the wood samples follow the same trend. However, applying higher pressure in the thermal treatment could cause cracks initiation in the middle lemalla and pits region, resulting in a more severe decrease of mechanical properties in bulk scale compared to the cell wall scale.

\subsection{Conclusion}

The Nanoindentation measurements revealed the local intrinsic wood cell wall properties, including hardness, storage modulus, loss modulus, and loss factor, which helped to gain deeper insights into the structural and compositional functions of the wood cell wall at the microscale.

Sample preparation, indent positions, and interpretation of nanoindentation test data were carefully considered to provide precise measurements.

The storage modulus showed strong positive correlations with loss modulus and hardness values, suggesting that they have a standard, deterministic origin.

It is suggested that local variations in cellulose fraction induce the strongly correlated scatter in the measured loss modulus and storage modulus of the wood cell wall. Indeed, where cellulose fibers and lignin are intended to increase the cell wall's stiffness and strength, the interfacial displacements, debonding, and friction between cellulose fibers and matrix could be identified as the fundamental energy dissipation mode in the cell wall.

It is shown that the local MFA variations cannot explain the positive correlation between $E^{\prime}$ and $E^{\prime \prime}$.

The loss factor is found to be relatively insensitive to local variations in the cell wall. As a result, it provides a sensitive metric for detecting changes regarding the externally imposed fields like thermal modification.

According to the thermal modifications applied on the pine wood, a systematic increase of elastic modulus and hardness was observed. However, the thermal treatment could not significantly affect the hardness and elastic modulus of eucalyptus wood. Despite that, a 
considerable reduction in the loss factor was observed for both pinewood and eucalyptus wood after thermal modification. It is proposed that hemicelluloses play an essential role in the overall damping performance of the wood cell wall before and after thermal treatment. 
742 MICROMECHANICAL MEASUREMENTS OF THE WOOD CELL WALL 


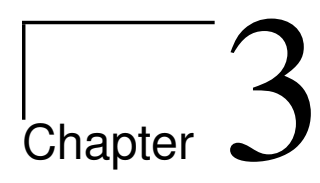

\section{Ultrastructural arrangement of the wood cell wall in nanoscale}

Wood, as a structural hierarchical material, has currently attracted scientist's attention to learn its genius pattern and generate new advanced materials in nanotechnology. To achieve this goal, detecting the physical properties and structural design of the wood cell wall assembly in nanoscale is one of the critical challenges. In this research, Atomic Force Microscopy (AFM), via a multifunctional mapping method, has been used to obtain the wood cell wall's topographical and compositional characteristics on the nanometer scale. The provided AFM compositional maps differentiated the secondary layers in the wood cell walls' transverse and radial sections. These determinations gave a better understanding of the spatial organization of the cell wall in three dimensions. Comparing the chemical and mechanical properties of the individual wood cell wall polymers, such as cellulose, lignin, and hemicellulose, is one of the elusive and critical issues in the wood study, which will be mentioned in this chapter. However, it still demands further research and development. 


\subsection{Introduction}

Wood as a natural hybrid material has an optimized structural design in all length scales, from the meter to the nanometer level. At least four different wood structure levels can be identified: Macroscopic structure including growth rings, microscopic structure, including cell wall and cellular design, nanostructure including secondary layers in the cell wall and molecular structure including individual polymeric building blocks like cellulose, lignin, and hemicellulose [8].

So far, considerable effort has been applied to investigate each building block individually and find their distribution in the cell wall. The wood cell wall included three main building blocks: cellulose, a linear polymer arranged in crystalline and amorphous shape, hemicellulose consisting of different polysaccharides, and lignin, an aromatic amorphous molecule.

According to our latest knowledge, the molecular structure of cellulose microfibrils consists of 18 cellulose chains representing the 3-4 $\mathrm{nm}$ fibrils visible by transmission electron microscopy $[16,166,167,168]$, that corresponds to the $2.5 \mathrm{~nm}$ fibrils observed by X-ray measurements $[169,170]$. These are the smallest fibril structures identified in cell walls, referred to as elementary fibrils [19, 171]. For the first time, the structure of cellulose microfibrils aggregate in the wood cell wall was explained by Frey-Wyssling in 1954 [11]. It was suggested that elementary fibrils could cluster together into microfibrils in various sizes. Until now, different techniques, including scanning probe microscopy, field emission scanning electron microscopy, and transmission electron microscopy, have been used to describe microfibril aggregates. These methods are often applied to develop cells [172], delignified fibers $[173,174,175]$, or decayed cell walls [176]. These studies have shown that microfibril aggregates are normal in chemically or physically altered cell walls, but little knowledge is available about cellulose aggregates in native cell walls [177]. In the research of Frey-Wyssling [19], the microfibril aggregates in chemically purified cellulose fibers were in the size of $250 \AA$. The study of Bardage et al. [174], indicated that cellulose aggregates in bleached pulp fibers are in the range of 18-20 nm, similar to the size found by Fengel [178]. Cellulose aggregation tendency during kraft processing was proposed to be the consequence of the lignin and hemicellulose exclusion from the cell wall [179, 180, 181]. 
Lignin is the next most prominent polymer in the cell wall. To fully comprehend the physical and chemical properties of lignin in the wood cell wall, the 3D structure of this macromolecule must be identified. Various electron-microscopic methods for analyzing the lignin distribution within the wood structure have been established till now. The distribution of lignin was determined by confocal laser scanning microscopy [182], ultraviolet (UV) technique [183, 184], and energy-dispersive X-ray analysis (EDXA) using bromination technique $[185,186]$. Furthermore, potassium permanganate $\left(\mathrm{KMnO}_{4}\right)$ was established as a general electron-dense staining agent for lignin [187]. Basically, the coniferylalcohol molecule is oxidized by $\mathrm{KMnO}_{4}$; therefore, indicating the reaction sites is possible when the permanganate anion is reduced to manganese dioxide and subsequently precipitates.

Among the different imaging techniques, atomic force microscopy (AFM) has been demonstrated as a robust and non-destructive method to track the wood cell wall's topography and mechanical properties in nanoscale. In recent years, several AFM studies have been applied to mostly embedded wood samples in contact [86, 87], and tapping mode $[6,188,189,113]$. The organization of secondary layers in the wood cell wall's crosssection and the distribution pattern of microfibril aggregates in the S2 layer was one of the main concepts in these studies. Fahlén and Salmén revealed a concentric lamellar organization of microfibrils in the wood cell wall [6, 188, 189] . In contrast, Zimmermann et al. [113] suggested that concentric and radial organization patterns coexist in the wood cell wall structure. They proposed a random organization of the microfibril aggregates. The aggregation of cellulose microfibrils in the S2 layer of softwood tracheids was reported in the size of 15 to $25 \mathrm{~nm}[6]$.

Recently, the AFM measurements have been performed on the wood samples to identify the middle lamella and secondary layers based on Young's modulus and stiffness gradient. However, nanoscale disclosure regarding the physical and mechanical properties of polymeric building blocks (e.g., cellulose, lignin) has not been represented so far [190, 191, 192]. Indeed the original structure of wood cell wall cross-sections is still debated, and further studies are necessary to demonstrate the nanoscale organization of the cell wall.

This study used bimodal Atomic Force Microscopy to investigate the native, non-embedded wood cell wall's ultrastructural design. In this method, the measurement of energy conservation and dissipation by a vibrating tip in the closeness of the sample surface provided 
the morphology and spatial compositional variations of the transverse and radial sections of the wood cell wall in ambient condition.

\subsection{Materials and Methods}

\subsubsection{Sample preparation}

For the experiments performed in this chapter, the radial and transverse sections of pine sapwood were prepared. Pine wood was chosen due to its relatively simple structure. Moreover, different plane sections of the cell wall were selected to have a better insight into the wood cell wall's secondary layers organizations from different perspectives of the wood samples. To have consistent AFM measurements on the wood samples, a perfectly smooth surface is required. At the same time, it is essential to do not to have any alteration in the sample's ultrastructure during specimen preparation. Therefore all forms of embedding and infiltration medium were avoided. Accordingly, the established method by Jakes et al. [96] was performed in this study. As shown in Figure 3.1, two unembedded wood specimens were prepared as pyramid-shaped by cutting with a razor blade. For the radial section preparation, the pyramid's apex was pointed perpendicular to the fiber axis and positioned in the latewood radial plane. And for the transverse section, the pyramid's apex was pointed parallel to the fiber axis and was placed in the latewood transverse plane. Afterward, $10 \mu \mathrm{m}$ slices were removed from the water brushed apexes using a Sartorius 31A30 sledge microtome equipped with a disposable steel knife. This procedure was concluded to an approximately $1 \times 1 \mathrm{~mm}^{2}$ smooth surface area for samples. Afterward, the samples were air-dried at room temperature for several days. The samples' moisture content was equilibrated in the AFM chamber at $65 \% \mathrm{RH}$ and $21^{\circ} \mathrm{C}, 12$ hours before $\mathrm{AFM}$ tests. 


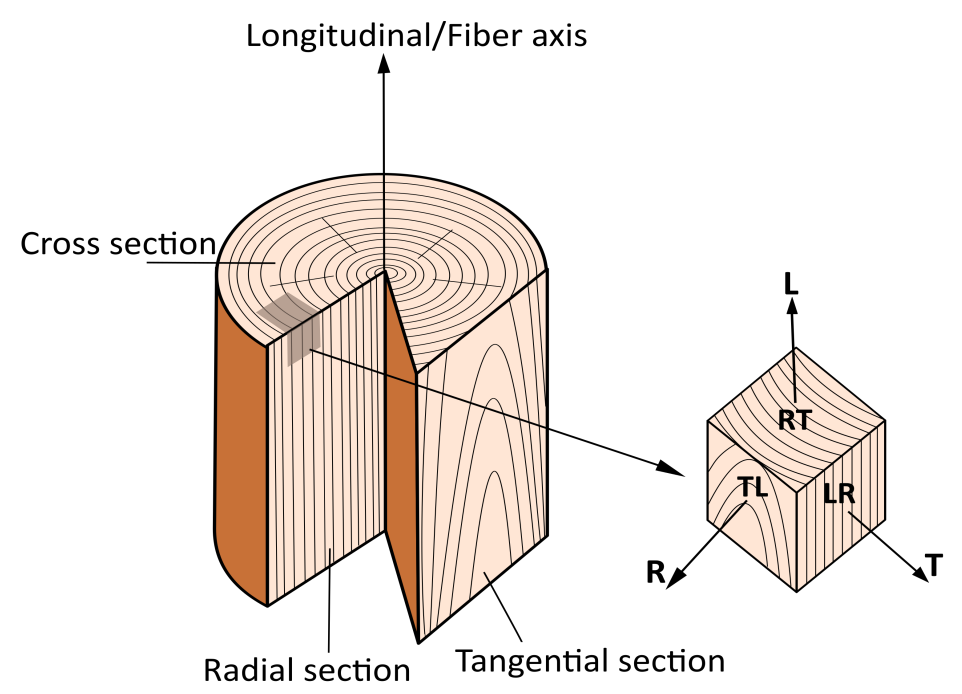

(a)

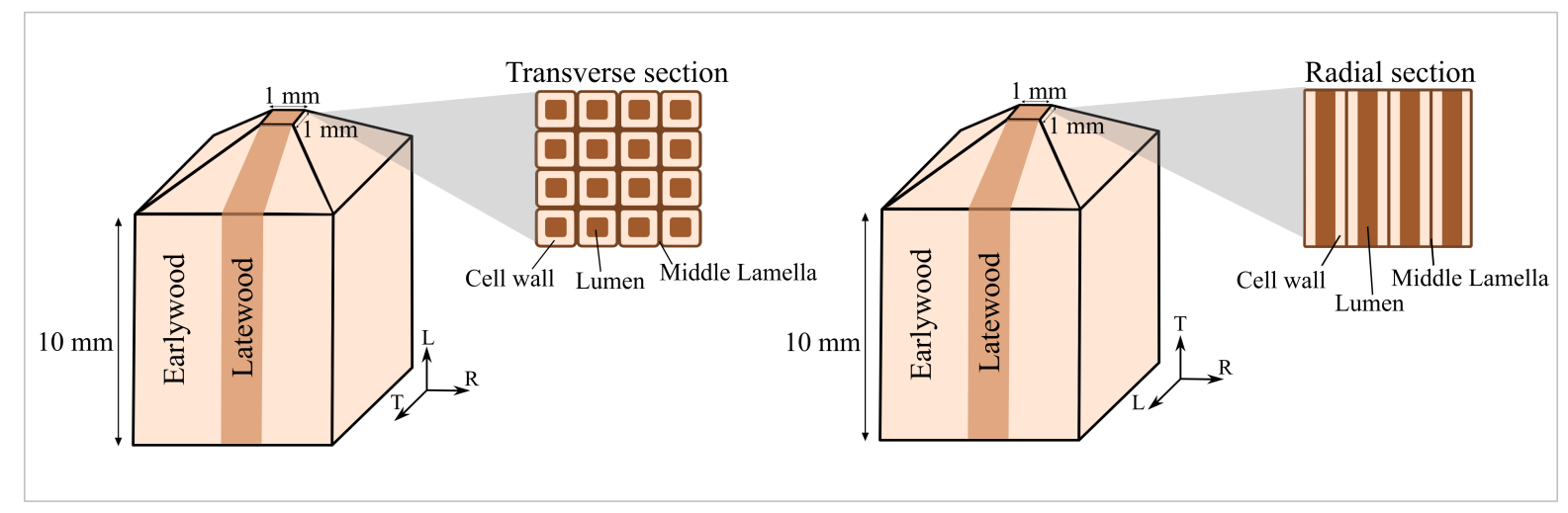

(b)

Figure 3.1: Schematic illustrations of (a) the longitudinal, radial, and tangential coordinate systems and plane sections of the tree trunk, (b) the pyramid-shaped samples positioned in the transverse and radial sections of the wood specimens.

\subsubsection{Atomic Force Microscopy method}

The ultrastructural organization of the wood cell wall in nanoscale was investigated with a commercial Atomic Force Microscopy (Asylum MFP 3D). The AFM method's fundamental concept is that a nanoscale tip attached to a bendable cantilever (so-called probe) interacts with the sample's surface over a raster scanning motion. In this work, we used the commercial silicon cantilevers (AC160TS) with the nominal stiffness of $26 \mathrm{~N} / \mathrm{m}$ and aluminum reflex coating. The cantilever included a crystalline silicon tip, where the tip 
height was $14 \mu \mathrm{m}$, and the tip radius at the apex was $10 \mathrm{~nm}$ nominally (see Figure 3.2).

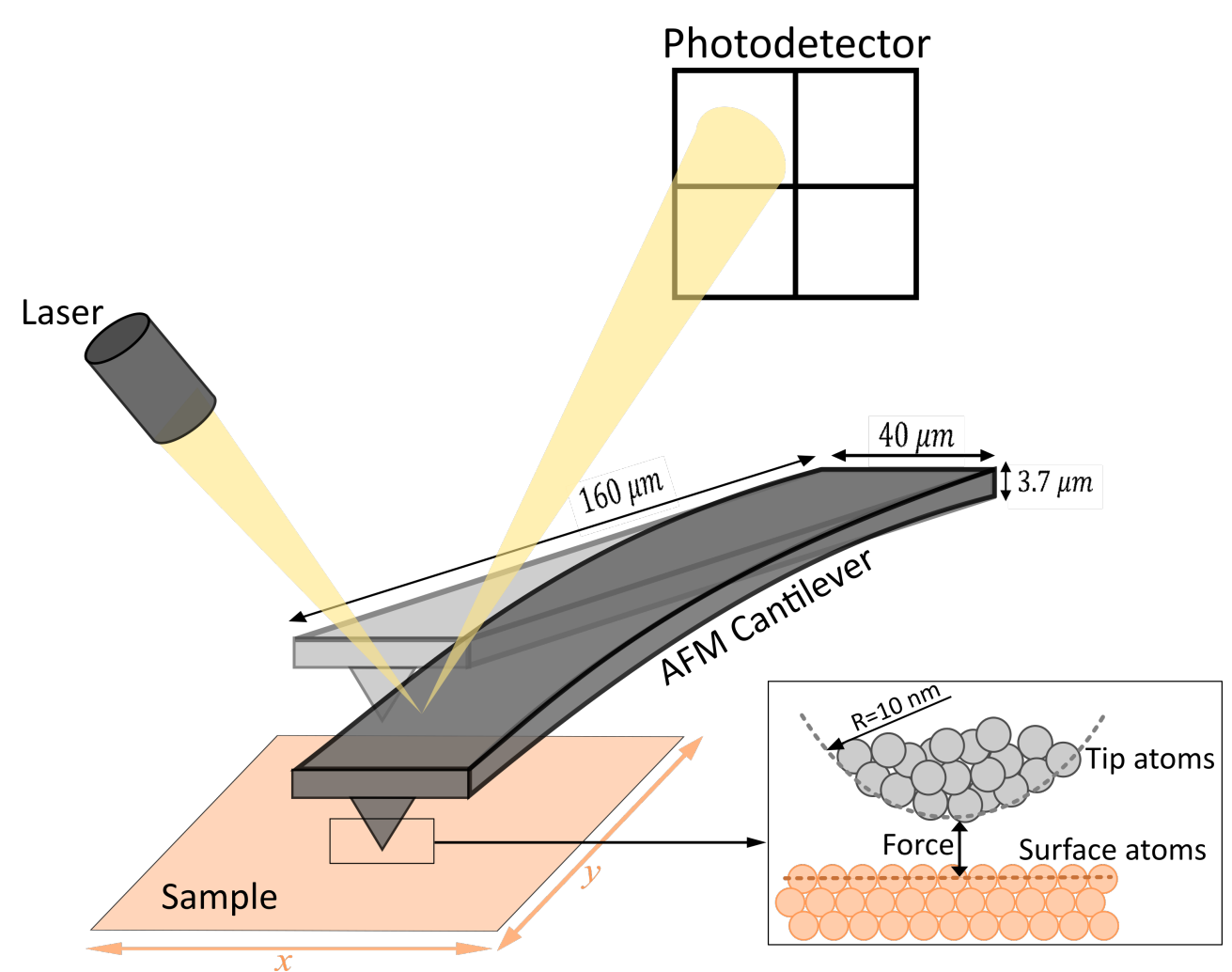

Figure 3.2: Schematic representation of the main components of the AFM concept. The deflection produced on the tip has reflected the photodetector with the laser beam.

The first mechanical principle of the AFM method is to measure the forces between atoms of the tip and the sample substrate. As a simple assumption, following Hooke's law (F = $\mathrm{k} \times \Delta \mathrm{z}$ ), when a probe with a spring stiffness $(\mathrm{k})$ approaches within some nanometers of a surface, the interacting forces $(\mathrm{F})$ between the tip and surface atoms cause a deflection $(\Delta \mathrm{z}=\mathrm{F} / \mathrm{k})$ in the probe. As the AFM tip scans the surface, a laser-reflection technique is used to measure the AFM tip's movement (up / down and side to side). The reflected laser beam is then tracked by a position-sensitive photodetector (PSPD) that picks up the probe's vertical and lateral motions (Figure 3.2). 


\section{Multifrequency dynamic atomic force microscopy}

In the current study, we used the dynamic AM-FM method. This method is selected because the high-resolution images (molecular and atomic resolution) are only achievable using very small oscillation amplitudes in the range of 1-2 $\mathrm{nm}$ in the dynamic mode rather than the static or contact mode [193]. Further, this method is non-destructive and can give even more information regarding the nanometer scale's energy-dissipation phenomena.

The Multifrequency AFM is based on the excitation of several eigenmodes' frequencies of the cantilever. Figure 3.3 shows the frequency response of a rectangular cantilever under a non-linear force. In this study, bimodal atomic force microscopy [194] was operated using a cantilever that was simultaneously driven at a superposition of the first and second of its resonance frequencies.

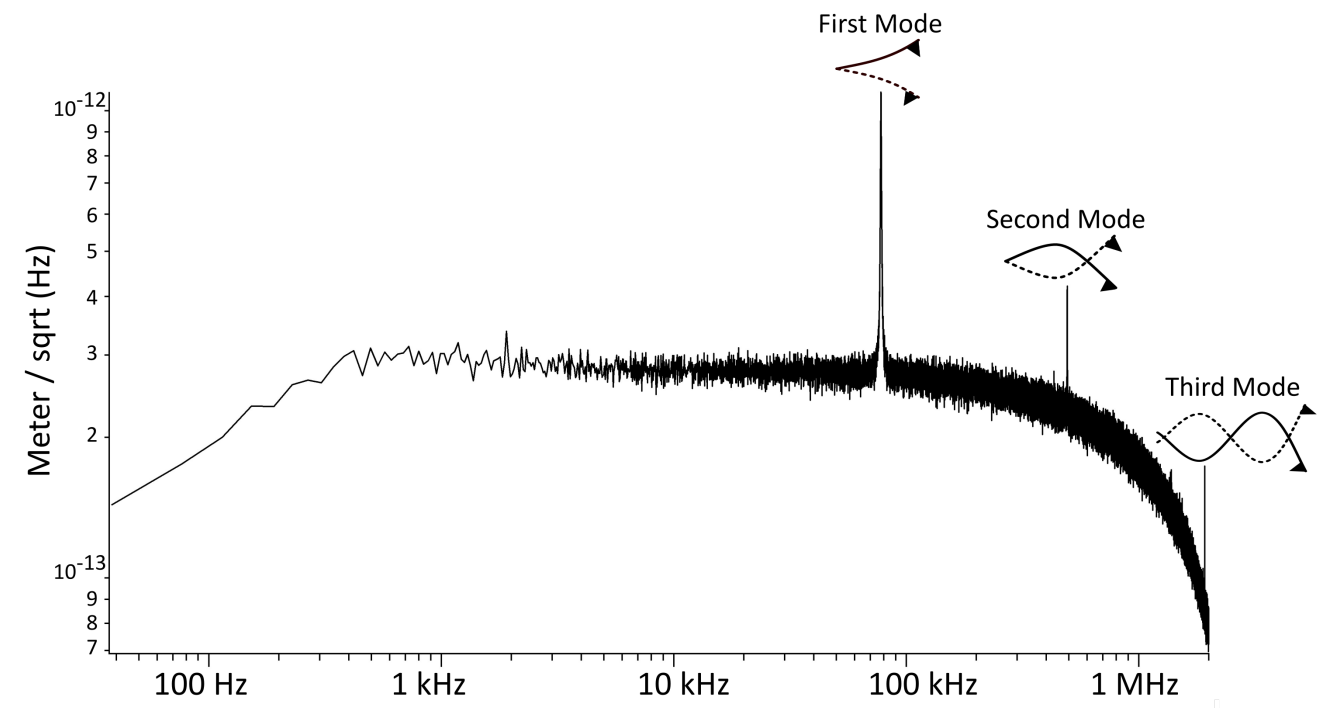

Figure 3.3: Typical thermal noise spectrum of a cantilever's deflection and its first, second, and third harmonic oscillating modes correspond to its first, second, and third resonance frequencies.

The principles of the AM-FM method, operated in the experimental setup of the current study, are described in Figure 3.4.

Before the tip-sample interaction, the first cantilever eigenmode was excited near its reso- 


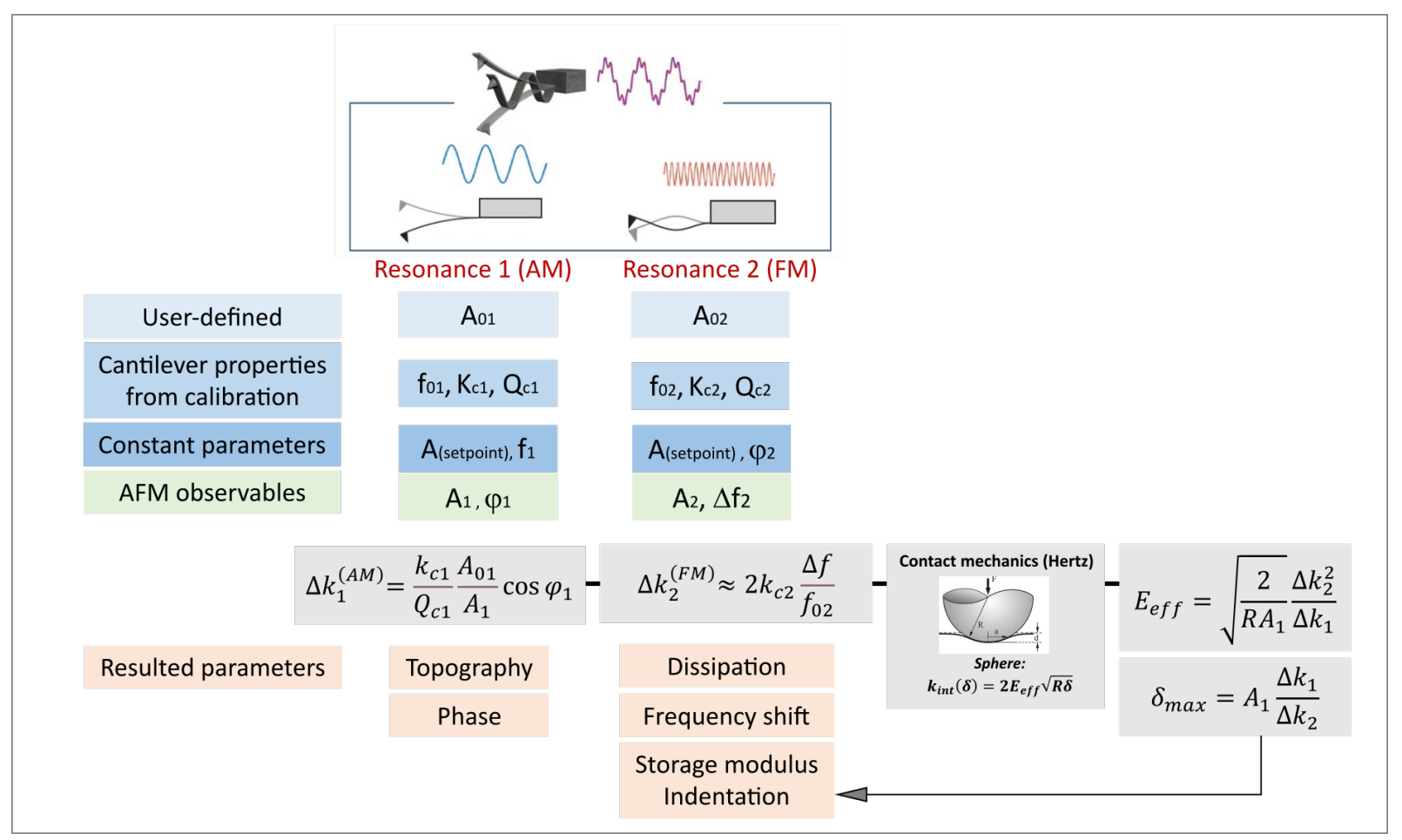

Figure 3.4: Schematic illustration for the bimodal AM-FM method. Resonance 1 operates in AM mode. The amplitude $A_{1}$ is the feedback parameter to provide topography, while changes in the phase lag $\left(\varphi_{1}\right)$ give values for loss tangent. Resonance 2 operates in FM mode. Changes in resonance frequency determine the stiffness and elasticity, while changes in the amplitude $\mathrm{A}_{2}$ provide viscous or dissipation information.

nant frequency $\left(f_{1}\right)$ with a large amplitude $\left(A_{01} \approx 2 \mathrm{~V}\right)$, where the second eigenmode was excited near its resonant frequency $\left(f_{2}\right)$ with a much smaller amplitude $\left(A_{02} \approx 25 \mathrm{mV}\right)$ simultaneously.

In continue, the AFM measurements' primary step included the cantilever stiffness calibration and the deflection sensitivity calibration. An automated routine in the MFP 3D instrument relied on the Sader method [195] was used to calculate the stiffness of the first eigenmode of the "free" cantilever away from the surface $\left(k_{c 1}=12.94 \mathrm{~N} / \mathrm{m}\right)$. The first eigenmode's stiffness was then used as a basis of calibration for the second eigenmode stiffness $\left(k_{c 2}=548.2 \mathrm{~N} / \mathrm{m}\right)$ [196]. Next, the cantilever's thermal noise spectrum was recorded [197] within a few hundred nanometers to the surface. A single harmonic oscillator model from the software was used to fit the thermal spectrum peak to detect the resonance frequency 
and quality factor of the first and second cantilever's eigenmodes $\left(f_{c 1}=204.81 \mathrm{kHz}, f_{c 2}=\right.$ $\left.1.33 \mathrm{MHz}, Q_{c 1}=244.094, Q_{c 2}=531.184\right)$.

Further, the deflection sensitivity calibration was carried out. Since the photodetector transforms the reflected laser beam in a voltage signal, this calibration is essential to change the voltage unit to the $\mathrm{nm}$ unit. Therefore, the equipartition theorem as an automated routine was used to convert both eigenmodes' amplitudes (in voltage unit) into nanometers unit [198, 199, 200]. This procedure provides a so-called InvOLS (Inverse Optical Lever Sensitivity) parameter in the $\mathrm{nm} / \mathrm{V}$ unit. In the current experiment, the measured InVOLS for the first and second modes were $39.88 \mathrm{~nm} / \mathrm{V}$ and $20 \mathrm{~nm} / \mathrm{V}$, respectively. It should be mentioned that the numbers mentioned above are typical values from one of the measurements.

Upon approaching the sample, both vibrations were controlled by two separate feedback loops (Figure 3.6), and consequently, multiple observables could be obtained regarding the tip-sample nanomechanical interactions. Certainly, the sample properties are figured from changes in a driven cantilever's parameters while interacting with the sample surface [201]. In the following section, we explain the fundamental operation of Amplitude Modulation mode (AM-AFM) and Frequency Modulation mode (FM-AFM) in details.

\section{First Mode: Amplitude Modulation (AM)}

In amplitude modulation mode, the cantilever is excited at its first resonance, where the oscillation amplitude is maximized. As the probe taps along the surface, the height variations in topography and the elastic/inelastic interaction forces between the tip and the surface change the amplitude and phase of the oscillation.

As shown in Figure 3.5, the cantilever oscillation amplitude is the feedback parameter to track the sample's topography in the AM mode. A specific setpoint (reduced target amplitude) for the amplitude is defined. The setpoint is represented as a percentage of the free amplitude $\left(A_{0}\right)$. Before scanning the surface where the tip is far from the surface, the amplitude is equal to the free amplitude $\left(A_{0}\right)$ (status 1 ). After approaching the surface, the oscillation amplitude reaches the set point amplitude value, and the tip starts scanning 
the surface (status 2). When the tip detects a change in the sample's topography, the oscillation amplitude changes (status 3), and this amplitude change is the source of the feedback. The feedback loop adjusts the tip-sample distance to maintain the setpoint amplitude at every image pixel by adapting the z-piezo to move the cantilever probe up and down (status 4). The resulting z-piezo movements provide the height information, so the scanned image represents the surface topography.

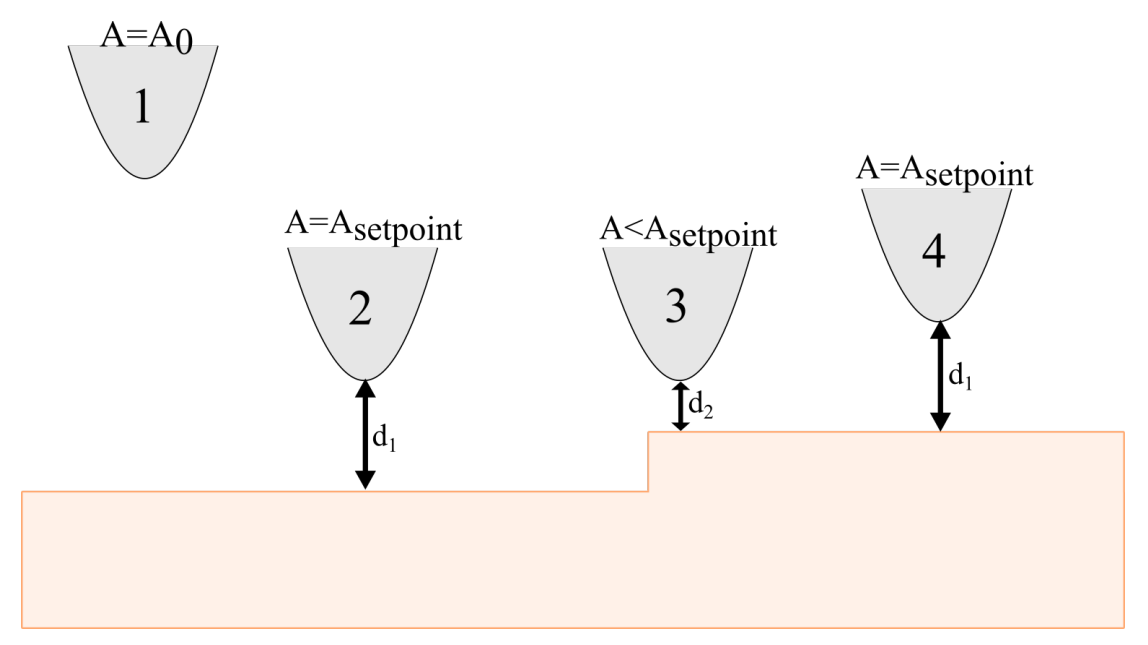

Figure 3.5: Schematic representation of the surface topography where the cantilever oscillation amplitude is the feedback parameter.

Another parameter collected with AM-AFM mode is the phase map that is simultaneously obtained with the topography map. At the resonance frequency, the phase shift is 90 degrees. During scanning the surface at a fixed amplitude, a phase shift occurs between the vibrating probe's excitation and its response to the tip-surface interactions. So the phase-contrast image provides a map of compositional variations of heterogeneous material.

Practically phase imaging is interpreted through the loss tangent of the tip-sample interaction. Although the phase map is a common map when contrast based on material properties is desired, measuring viscoelastic properties in nanoscale is generally complicated due to the phase dependence on near-surface effects. It is mentioned in the works of literature that the phase response depends not only on the energy transferred from the tip to the sample surface (dissipated energy) but also on the strength of the tip-sample conservative interactions (stored energy) [202]. Furthermore, some dissipation sources, such as the surrounding air environment, absorbed water on the sample and tip, and sample 
plasticity can lead even to overestimations in the dissipated energy and underestimations in the stored energy [203]. So the phase shift happens due to the complexity of multiple material properties, such as adhesion, dissipation, viscoelasticity, and stiffness, and it can be challenging to interpret the phase contrast and refer it to the material's individual property.

Indeed, in tapping AM mode, only the ratio of the loss modulus to storage modulus can be measured as the phase map. Separating the storage and loss moduli requires additional independent observables that can be extracted by the FM-AFM mode.

\section{Second Mode: Frequency Modulation (FM)}

In FM mode, the cantilever is excited at an additional flexural mode. By performing this mode, the tip-sample contact's mechanical parameters, such as reduced elastic modulus $E$, dissipation energy, and indentation depth $\delta$, can be calculated. When the tip approaches the sample, the tip-sample interaction is inducing a decrease in the oscillation amplitude and a shift in the resonance frequency.

The dissipation map originates from changes in the second amplitude $\left(A_{2}\right)$. An amplitude feedback loop adjusts the drive voltage to keep the second amplitude constant. The output of the second voltage signal contains information on viscous or dissipative forces. Basically, the energy which is fed into the cantilever will dissipate from two different sources [138]: Background dissipation, which could come from the body of the cantilever, like air damping. Tip-sample dissipation interaction includes localized dissipation between the tip and sample.

The resonant frequency changes are also recorded on FM mode, while the phase shift between the driving signal and the response is kept $90^{\circ}$ by a phase-locked-loop (PLL) [194]. The output of the second resonance frequency describes the elastic tip-sample interaction. Basically, higher frequency means greater stiffness or modulus. The frequency shift can then be converted into quantitative Young's modulus values through the Hertz-Sneddon model using a series of equations outlined in the next section. 


\section{Contact mechanics in the AM-FM method}

In the bimodal AM-FM method, experimental observables' transformation into nanomechanical properties is accomplished by using suitable contact mechanics models. In the current study, the Hertzian contact model was chosen as the simplest model, which was available in the latest version of the MFP-3D software from the Oxford Instruments company. In the Hertzian contact model, the interaction stiffness kint is defined regarding the indentation depth $\delta$, the tip radius $R$, and effective Young's modulus. As shown in Figure 3.6, by assuming to have a paraboloid (sphere) shape at the apex of the tip and having a flat surface of the sample, the interaction stiffness kint is calculated as below [126]:

$$
k_{\text {int }}(\delta)=2 E_{\text {eff }} \sqrt{R \delta}
$$

The tip radius $R$ was assumed to be at its nominal value of $10 \mathrm{~nm}$. The effective Young's modulus $E_{\text {eff }}$ combines deformation of the tip and sample [126]. Since the probe Young's modulus $\left(E_{t i p}\right)$ is at least two orders of magnitude larger than Young's modulus of the wood sample studied here, the effective Young's modulus is assumed to be equal the sample Young's modulus $\left(E_{\text {eff }} \approx E_{s}\right)$.

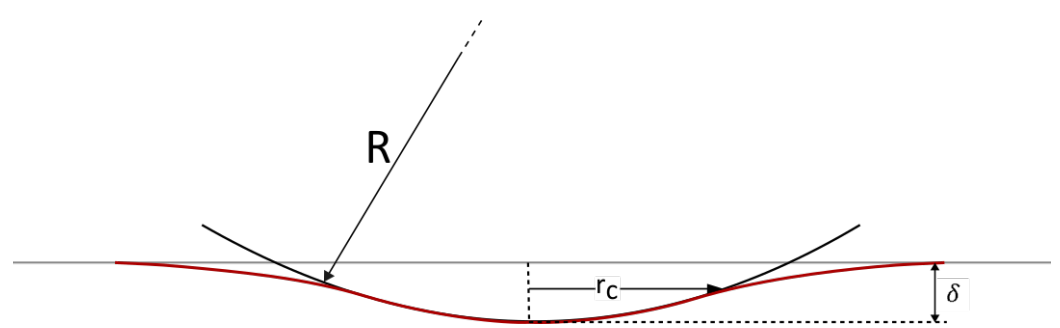

Figure 3.6: The indentation of a paraboloidal indenter with radius $R$ into an elastic surface [126].

In the bimodal interaction theory, the tip-sample interaction stiffness $\left(k_{i n t}\right)$, which is the instantaneous stiffness profile experienced by the oscillating cantilever tip, affects the timeaveraged change in stiffness $(\Delta k)$ of the cantilever tip-sample system. In this method, $\Delta k$ is experimentally measurable in the cantilever's first and second eigenmodes [204]. For 
the first and second eigenmodes, the change in stiffness of the interacting cantilever $\left(\Delta k_{1}\right.$, $\Delta k_{2}$ ) can be computed by integrating $k_{\text {int }}(\delta)$ over one cycle, with a semi-circular weight function [205]. The mathematical calculations are presented in detail by the work of Labuda et al. [204]. By taking into account that the first eigenmode is driven at amplitude A1 which is considerably larger than the interaction length scale, and the second eigenmode is purposely driven at a small amplitude $A_{2}$, such that the interaction stiffness is roughly constant through one cycle of the higher eigenmode oscillation, the weight function can be approximated, and the time-averaged change in interaction stiffness of the first and second eigenmodes $\left(\Delta k_{1}, \Delta k_{2}\right)$ can be calculated as below:

$$
\begin{aligned}
& \Delta k_{1} \approx \sqrt{\frac{R}{2 A_{1}^{3}}} E_{e f f} \delta_{\text {max }}^{2} \\
& \Delta k_{2} \approx \sqrt{\frac{R}{2 A_{1}}} E_{e f f} \delta_{\max }
\end{aligned}
$$

By the combination of Equations 3.2 and 3.3, the following analytical expressions of the maximum indentation depth $\left(\delta_{\max }\right)$ and the effective Young's modulus $\left(E_{\text {eff }}\right)$ are extractable as a function of the interaction stiffness change $\left(\Delta k_{1}, \Delta k_{2}\right)$ :

$$
\begin{aligned}
\delta_{\max } & =A_{1} \frac{\Delta k_{1}}{\Delta k_{2}} \\
E_{\text {eff }} & =\sqrt{\frac{2}{R A_{1}}} \frac{\Delta k_{2}^{2}}{\Delta k_{1}}
\end{aligned}
$$

moreover, for the first eigenmode driven in AM mode, the corresponding time-averaged interaction stiffness $\left(\Delta k_{1}\right)$ can be calculated from the measured interaction amplitude $A_{1}$ and interaction phase $\varphi$ as follows:

$$
\Delta k_{1}^{(A M)}=\frac{k_{c 1}}{Q_{c 1}} \frac{A_{01}}{A_{1}} \cos \varphi
$$


where the cantilever quality factor $Q_{c 1}$ the cantilever stiffness $k_{c 1}$, and the reference amplitude $A_{01}$ (free amplitude) are all measured during the calibration procedure for the first eigenmode.

In the second eigenmode driven in FM mode, where the resonance frequency $f_{2}$ is tracked with a phase-locked-loop (PLL), the measured frequency shift $\Delta f$ can be used to estimate the interaction stiffness $\left(\Delta k_{2}\right)$ by estimation as bellow:

$$
\Delta k_{2}^{(F M)} \approx 2 k_{c 2} \frac{\Delta f}{f_{02}}
$$

Consequently, combining equations 3.4, 3.5, 3.6, and 3.7, the $\delta$ and $E_{\text {eff }}$ are extractable related to the experimental observables. In Figure 3.4, the whole procedure for the AM-FM method has been summarized.

\subsection{Results and Discussion}

\subsubsection{AM-FM maps configuration for the wood cell wall}

In Figure 3.7, an example of provided maps from the AM-FM methods is presented. The topography and materials property maps of the wood cell wall cross-section are shown, which all were taken simultaneously during one AFM measurement. The different interaction resonances act as different channels representing separate material properties such as topography, viscoelasticity, elasticity, and the indentation depth.

The AFM topography (Figure 3.7(a)) shows a granular structure of the S2 layer in the wood cell wall's transverse section. According to the root mean square value of the height irregularities, the surface roughness is $3.49 \mathrm{~nm}$, which confirms that the topography map has a reasonably smooth surface suitable for mechanical measurements. It should be noted that the horizontal scratched lines are due to the stick-slip motion of the knife blade.

The surface's granularity is also preserved in the phase, frequency shift, elastic modulus, and indentation maps (Figure 3.7(b-e)). We call these maps materials property maps, 
First Mode:

Amplitude Modulation (AM)

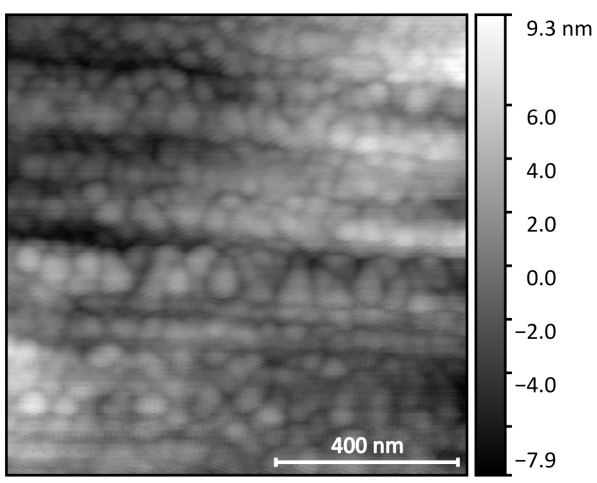

(a)

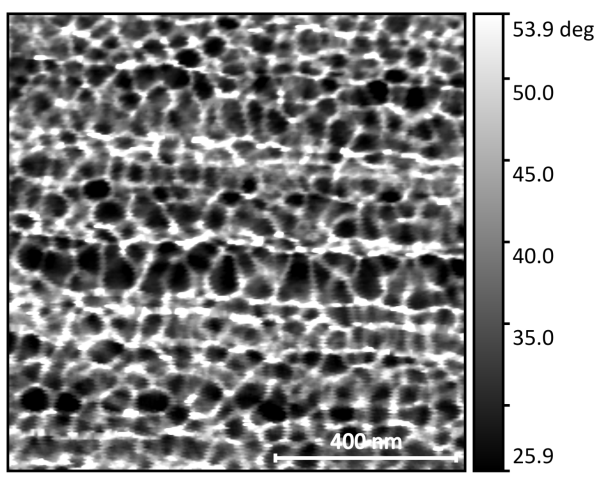

(b)
Second Mode:

Frequency Modulation (FM)

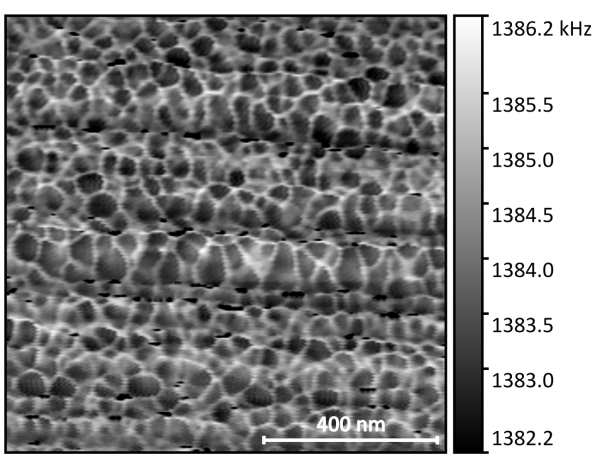

(c)

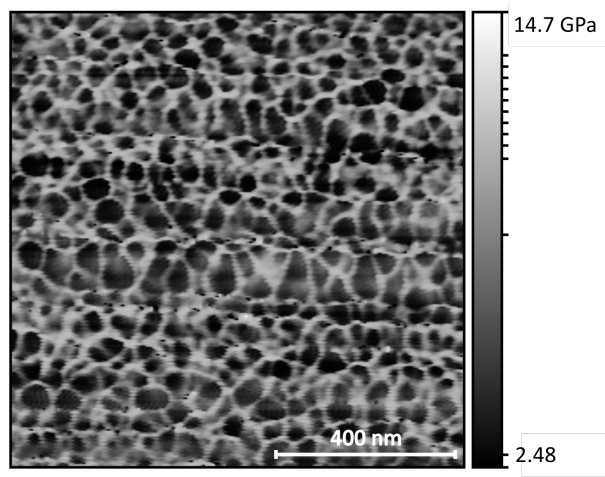

(d)

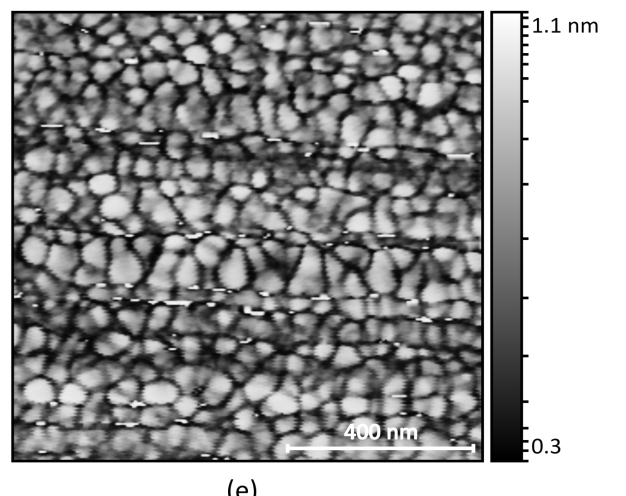

(e)

Figure 3.7: Ultrastructural arrangement of the S2 layer in the transverse section of the wood cell wall. (a) topography, (b) Phase map, (c) Frequency map, (d) Modulus map, and (e) Indentation depth map.

which show significant contrast between the wood cell wall polymers and reveal a structure composed of ca. $30 \mathrm{~nm}$ diameter inclusions embedded in a matrix. 
In the phase map (Figure 3.7(b)), the inclusions have a small phase lag, while the matrix has a high phase lag. Basically, phase imaging is interpreted regarding the loss tangent of the tip-sample interactions. However, practically the phase change occurs due to the complexities of several material properties, such as adhesion, dissipation, viscoelasticity, and stiffness. Therefore, it is not easy to refer the phase contrast to an exclusive property of the material. It has been shown that under ambient conditions, capillary forces influence the phase contrast in repulsive mode [206, 207]. Water condensation can create a nanometersized water bridge as the tip approaches the sample. So brighter phase lag may exhibit a more hydrophilic surface [206]. The lignin and cellulose molecule's chemical models indicate that lignin has two hydroxyl groups per 10 carbon atoms, while there are $3 \mathrm{OH}$-groups per 6 carbon atoms in the polysaccharides [178]. That means the lignin molecules are less hydrophilic than the polysaccharide molecules. Considering the cellulose's hydrophilic behavior in the wood cell wall, the light area in the phase map could be related to cellulose. The phase map, shown in Figure 3.7(b), is consistent with the adhesion force map of recent researches [208, 209]. Felhofer et al. suggested that the hydrophilic polysaccharides and hydrophobic lignin can be differentiated regarding the silicon tip's tendency to hydrophilic polysaccharides [208].

The frequency map and Young's modulus map (Figure 3.7(c,d)) also reveal two different domains on the sample surface: the circular areas (softer) and the surrounding matrix (stiffer) domains, which is consistent with the modulus maps of other studies [190, 191, 209]. Based on the fact that the elastic modulus of cellulose ( $\sim 130 \mathrm{GPa})$ is much higher than that of lignin ( $\sim 3 \mathrm{GPa})$ [4], it is suggested that presumably the matrix is cellulose and the inclusions are aggregates of lignin molecules.

These results indicate that the cell wall's ultrastructural arrangement is composed of lignin inclusions in a cellulose matrix that contrasts with what has long been assumed and is still intriguing for further investigations.

As one explanation, it is likely that the inverted modulus results could be due to the artifact in AFM measurements in nanoscale! It is well known that despite the advantages of the AFM method, the probing ability of AFM is limited when used for surfaces that have features comparable or smaller than the tip radius [210]. 
Changes in the surface design by cutting is another possible interpretation. So the surface structure (including topography) which we measure could be the product of the cutting process. It is possible to adjust the structure by cuts, and that this could account for the high correlation in Fig. 3.7.

More discussion about the possible model for cell wall ultrastructure is proposed in Appendix B. Appendix $\mathrm{C}$ is also available in which the author provided more investigation on cellulose and lignin distribution throughout the cell wall with various treatments.

It should be mentioned that the aim of Young's modulus map presented here is providing high-resolution qualitative images rather than quantitative values. In fact, because of the simplifying hypothesis of the Hertzian contact model, the numbers given in the elastic modulus map's color scale bar are taken into account qualitatively, and we do not discuss the absolute values of Young's modulus of the circular areas and the surrounded matrix in this chapter.

The indentation map also depends on the sample's local mechanical properties (Figure 3.7(e)). The relative stiff matrix shows smaller indentation, while the indentation values are larger for the more compliant inclusion regions. So the contrast in the indentation and Young's modulus maps are consistent with each other.

\subsubsection{The effect of topography}

Identifying the topography's role in compositional maps is often a controversial topic in atomic force microscopy measurements. The effect of local roughness on nanomechanical measurements, especially elastic modulus, may not be ignored and is still under debate between researchers. In fact, it might be challenging to achieve full contact of the tip in the valley areas, and the contact area may be significantly reduced which consequently, causes the overestimation of elasticity in these areas. For this reason, we tried to check out the dependency of compositional maps on the topography to find out a fair interpretation of the presented AFM maps in this study.

As shown in Figure 3.8, the plotted phase, modulus, and frequency versus height diagrams gained from corresponding maps in Figure 3.7, do not show a clear correlation between 
compositional maps and topography.

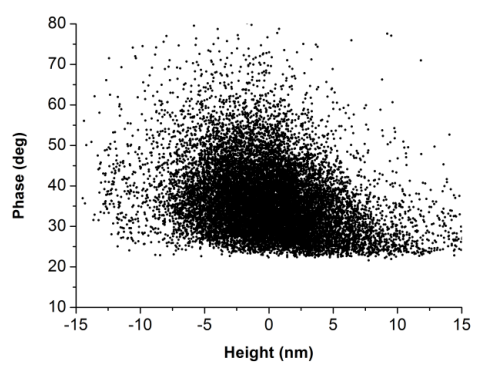

(a)

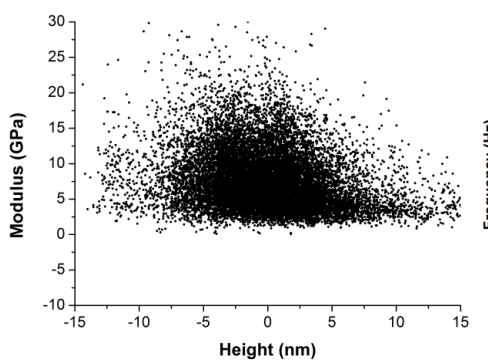

(b)

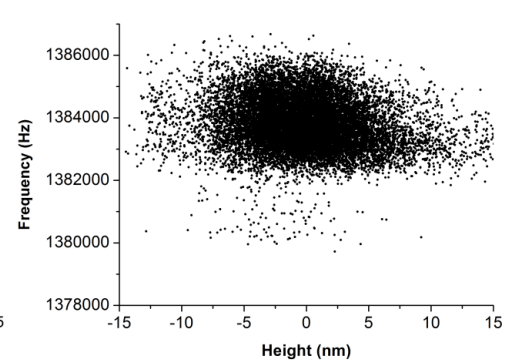

(c)

Figure 3.8: Topography correlation with (a) Phase map, (b) Modulus map, and (c) Frequency map gained from corresponding maps in Figure 3.7.

Further, to investigate the effect of the surface curvature on the elasticity, we calculated the curvature of the surface from the topography map in Figure 3.9(a), included $512 \times 512$ scan points in $2 \mu \mathrm{m} \times 2 \mu \mathrm{m}$ area. After that, the distribution of modulus values (Figure 3.9(b)) was evaluated corresponding to the different surface curvature values. As shown in Figure 3.10, the surface curvature calculation was implemented according to the height of 3 neighboring points $(i-1, i, i+1)$ with a pixel's distance $(p)$.

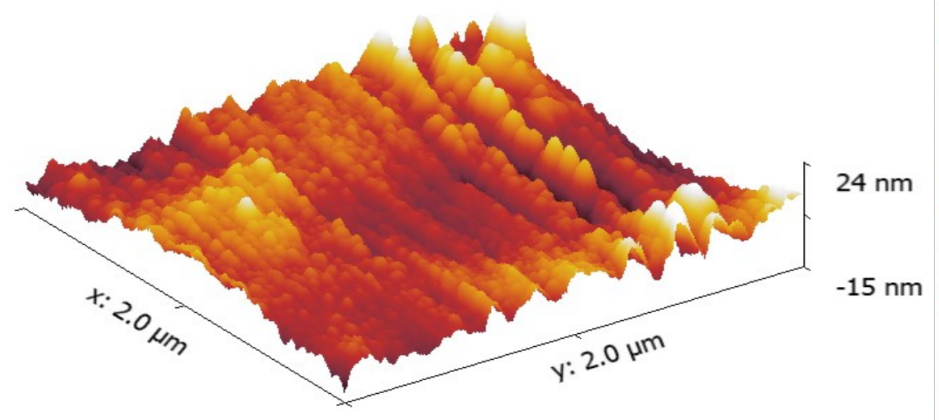

(a)

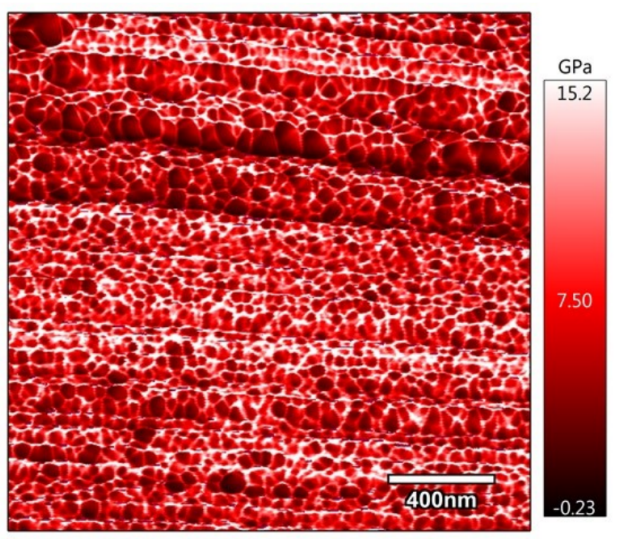

(b)

Figure 3.9: (a) 3D representation of the height map and (b) modulus map, included $512 \times 512$ scan points in $2 \mu \mathrm{m} \times 2 \mu \mathrm{m}$ area. 


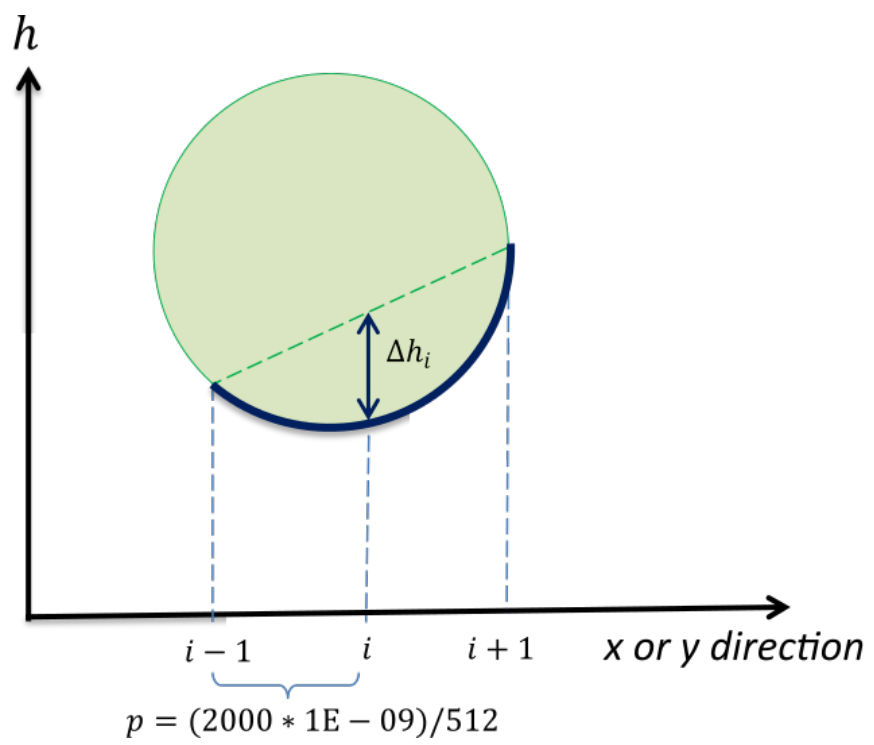

$$
\begin{aligned}
\Delta h_{i} & =h_{i-1}+\frac{1}{2}\left(h_{i+1}-h_{i-1}\right) \\
\Delta h_{i} & =\frac{1}{2}\left(h_{i+1}+h_{i-1}\right) \\
R_{i} & =\frac{p^{2}+\Delta h_{i}^{2}}{2 \Delta h_{i}} \\
K_{i} & =\frac{1}{R_{i}}
\end{aligned}
$$

Figure 3.10: 2D curvature of a line. Curvature $(K)$ is defined as the inverse of the radius of a circle tangent to the surface of three neighboring points

The curvature values are then categorized into three groups (Figure 3.11): group (a), where the surface's curvature is close to zero in the flat areas. Group (b), where the curvature is negative in the concave valley areas. Group (c), where the curvature is positive in the convex hills areas. As shown in Figure 3.11(a), the elastic modulus histogram diagram in flat areas where the surface's curvature is very close to zero is in the range of 0-24 GPa. This elastic modulus distribution is almost the same in the valley areas with positive curvature (Figure 3.11(b)). It means that the distribution of elastic modulus on flat surfaces and in the valley areas is almost the same, and the valley area does not show a significant effect on the contact area and elastic modulus measurements. The negative curvature areas, which refer to the high hills of the globular structures, show a lower elastic modulus (Figure 3.11(c)). One possible explanation for reduced modulus at the top of the hills is the reduction in lateral constraint (topography effect). Another possible explanation is the property of the material, where granular aggregates of lignin are assumed to be the result of the cutting process in producing the high hills.

Comparing the counts of the scanned points in the histograms shows that there are much 
(a)

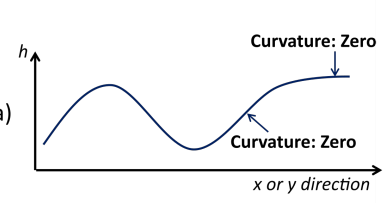

(b)
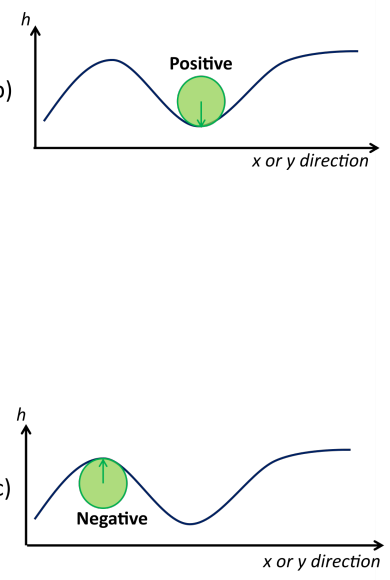
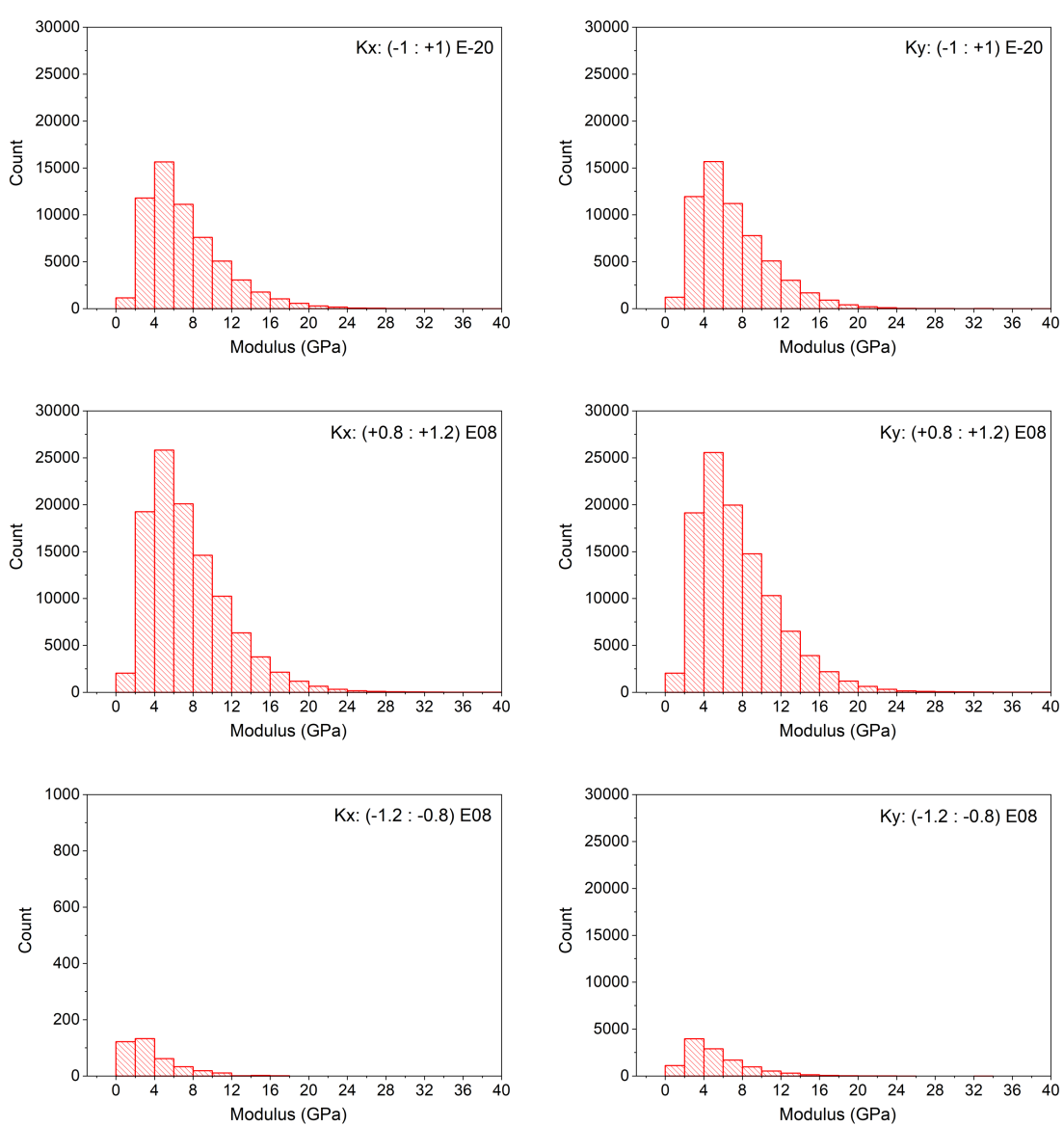

Figure 3.11: The histogram diagrams of modulus distribution regarding the: (a) flat areas, surface curvature is close to zero, (b) concave valley areas, positive curvature, (c) convex hill areas, negative curvature. The surface curvature calculation is performed for both $x$ and $y$ directions in the height map.

fewer measurements in the high hill areas (Figure 3.11(c)), in comparison to the flat (Figure 3.11(a)) and valley areas (Figure 3.11(b)). It is because the hills are mostly flat on top and go to the flat surface category rather than the hill category.

Evaluating the number of counts in the histogram of these three categories show that about $96.48 \%$ of the measurements belong to the flat and valley area that show the same distribution of elastic modulus, and 3.5\% of the measurements belong to the high hill areas with lower modulus distribution. This shows that about $96 \%$ of the measurements are independent of the surface curvature, providing evidence that the contrast of the elastic modulus 
map is mainly due to the variation of material stiffness rather than surface roughness.

\subsubsection{Comparing different regions of the wood cell wall}

This section presents AFM images of the wood cell wall's radial and transverse surfaces to better understand the microfibril spatial organization in secondary layers. Since we aimed to look into the cell wall's morphology, the AFM maps with the best resolution were our criteria to choose, to see as many details as possible in the structure. Therefore, different radial and transverse surfaces, from frequency map, phase map, or dissipation map, were chosen rather than the topography map.

\section{Microfibrils structure in the S2 layer}

Figure 3.12 shows the S2 layer of the transverse and radial sections of the pinewood cell wall provided with atomic force microscopy. It is believed that the wide S2 layer, which forms about 80 to 90 percent of the wood cell wall, consists of tightly packed microfibrils arranged at an angle of $0^{\circ}$ to $20^{\circ}$ degrees [8]. In the shown $\mathrm{S} 2$ layer of the radial and transverse sections, inclusions are visible in a globular structure. The inclusions' structures in the cross-section map (Figure 3.12(b)) are almost in the round shape, regarding the fact that the sectioning plane is perfectly perpendicular to the fibers. In the radial section, the inclusions' structures are mostly in the larger round shape and elliptical shape (Figure 3.12(a)), which could be related to the fibrils' helical orientation around the wood cell wall and deviation of sectioning/cutting angle from $90^{\circ}$ concerning the direction of the fibers. 


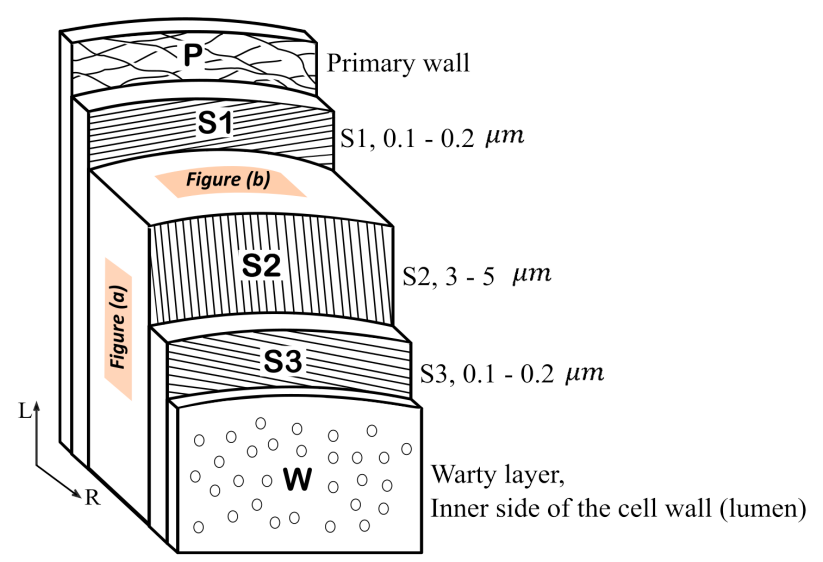

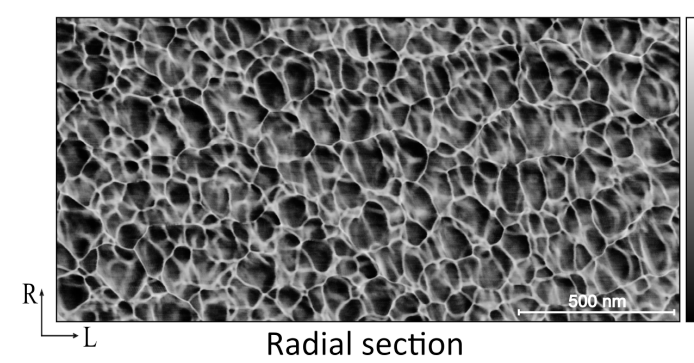

(a)

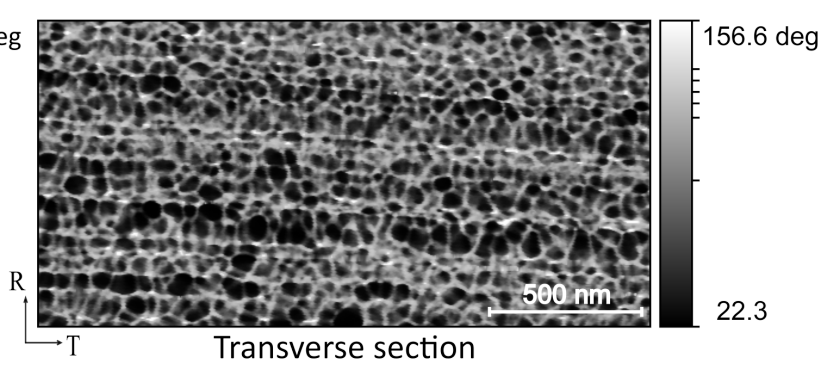

(b)

Figure 3.12: Comparing the AFM images of the (a) radial section and (b) transverse section of the S2 layer in the wood cell wall. The highlighted areas in the schematic picture of the cell wall show the corresponding scanned regions.

\section{Microfibrils structure in the $\mathrm{S} 1$ and $\mathrm{S} 2$ region}

Figure 3.13(a) shows the schematic picture of the wood cell wall's radial section and secondary layers, where the highlighted area in the picture refers to the corresponding scanned area of the cell wall with atomic force microscopy.

Figure 3.13(b) shows the AFM image of the pinewood cell wall's radial section, where the $\mathrm{S} 1$ layer close to the middle lamella and the broad region of the S2 layer are visible. In the upper part of Figure 3.13(b), the microfibrils are in a nearly parallel view. This area refers to the S2 layer as the thickest cell wall layer with approximately $5 \mu \mathrm{m}$ width. In the bottom part of Figure 3.13(b) near the ML, the microfibrils are in an almost cross-sectional view that might refer to the S1 layer where the microfibrils are perpendicular to the cell 


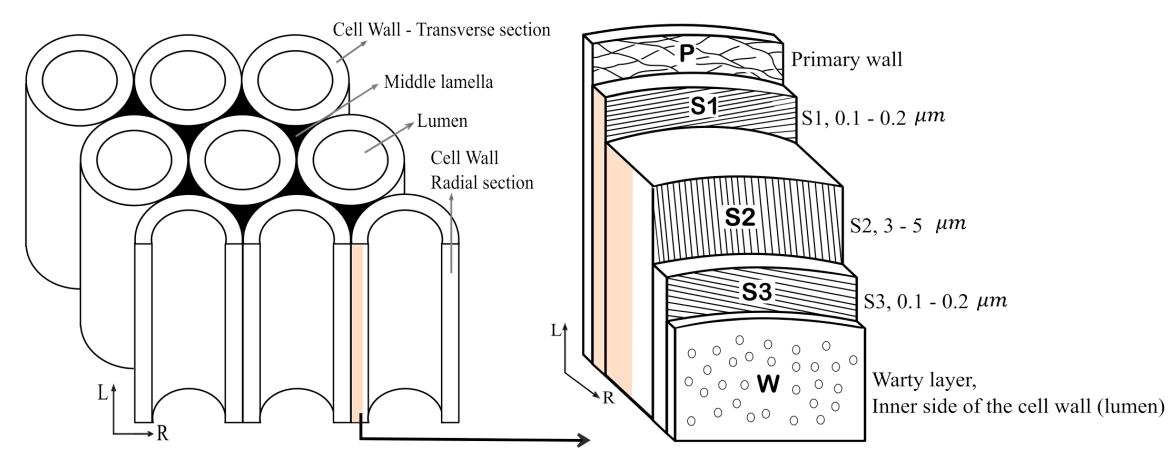

(a)

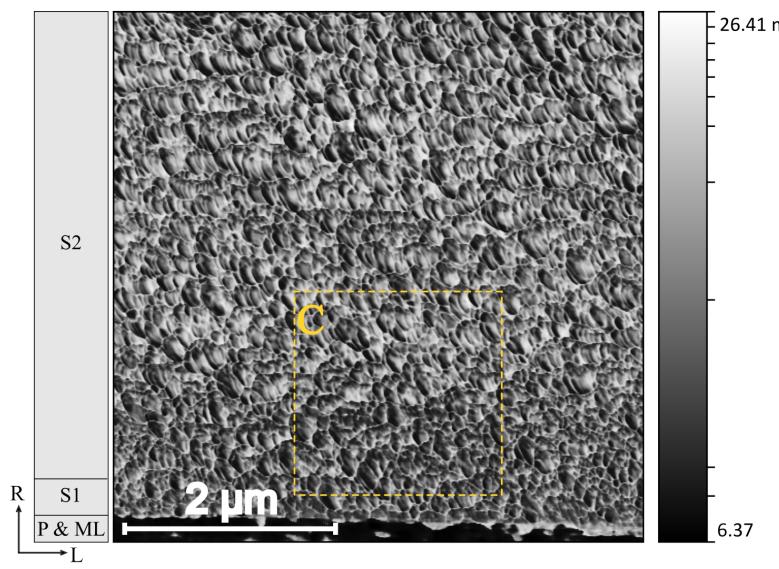

(b)

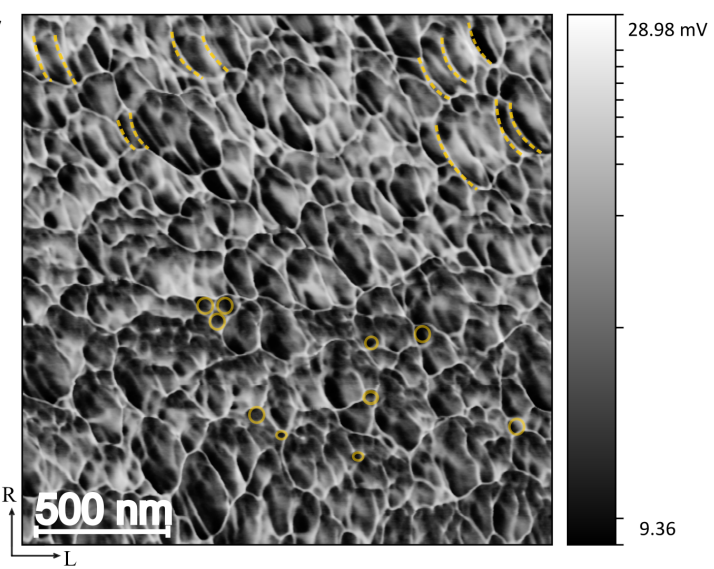

(c)

Figure 3.13: (a) Schematic picture of the wood cell wall's radial section and secondary layers. The highlighted area shows the scanned area with atomic force microscopy, (b) Dissipation map of the radial section, related to the S2 and S1 layers (c) Higher magnification of the marked area in figure b.

axis [8].

The different configurations are shown in Figure 3.13(c) with higher magnification. This appearance is not unexpected by considering the microfibrils' helical orientation around the fiber axis and the microfibrils angle change between the S1 and S2 layers. The different color coding in Figure 3.14 shows the structure alteration from the middle lamella to the S2 layer more clearly.

Figure 3.15 shows the cross-sectional view of the spruce wood cell wall, where the morphology of the middle lamella, S1 layer, and S2 layer is visible. ML has a randomly arranged 


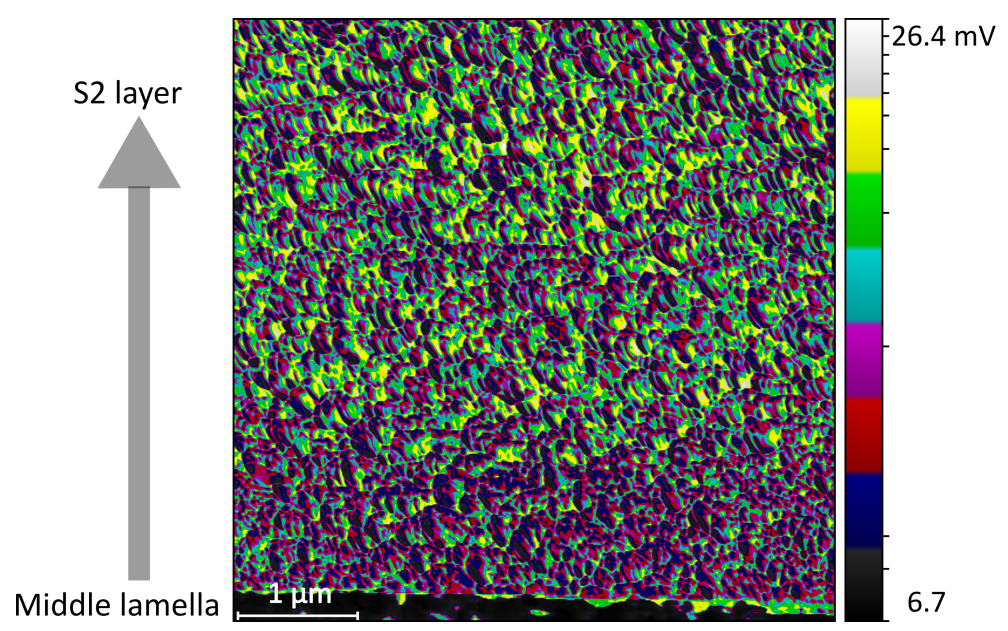

Figure 3.14: The illustration of the morphology change from the middle lamella to the S2 layer, using different color coding of dissipation map.
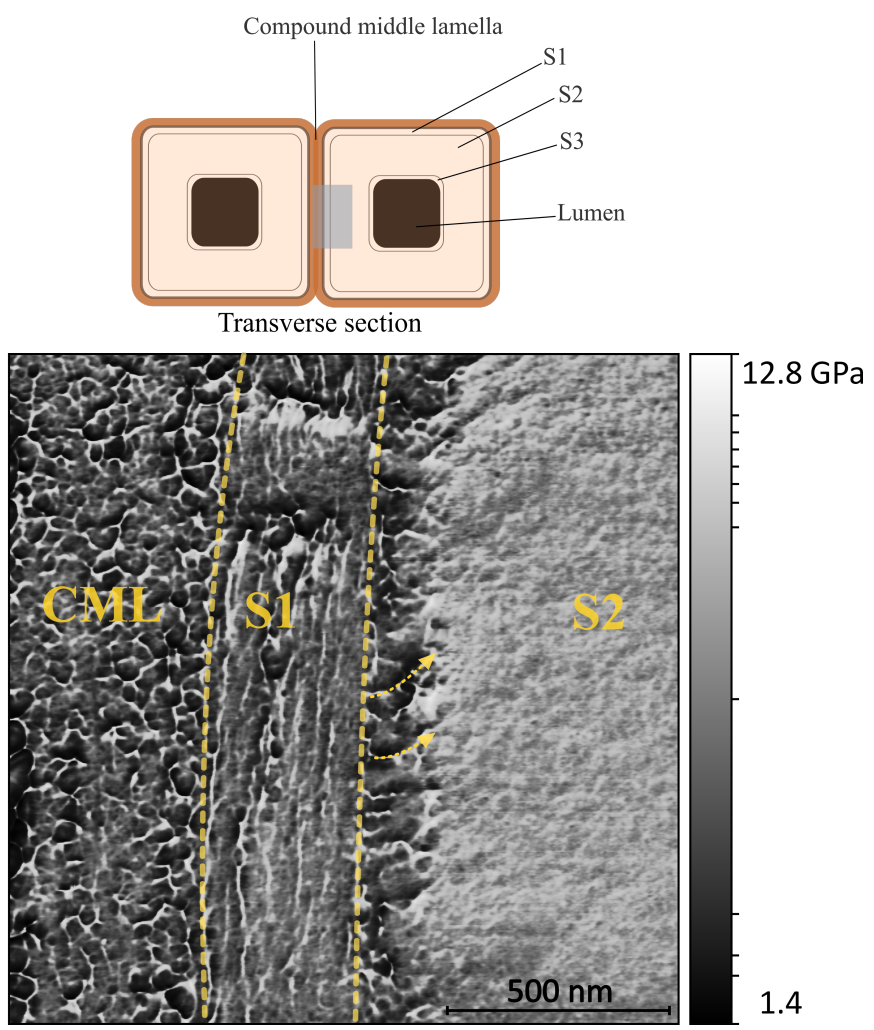

Figure 3.15: The AFM image of the spruce wood cell wall's cross-section. The ML, S1, and S2 layers are shown in the figure. The radial orientation of the microfibrils from the $\mathrm{S} 1$ layer towards the $\mathrm{S} 2$ layer is visible. 
globular structure of lignin. In the S1 layer, the fibrils are oriented in a parallel view perpendicular to the cell axis, while the $\mathrm{S} 2$ layer shows a very firm and dense structure. The radial direction of microfibril from the S1 layer towards the S2 layer also is apparent.

Regarding the AFM elastic modulus map in Figure 3.15, the S2 layer shows higher elastic modulus in comparison to the S1 and ML layers, which is reasonable. The higher concentration of lignin in ML and S1 layers shows a lower elastic modulus, while the higher concentration of cellulose in the S2 layer shows higher stiffness in the AFM elastic modulus map. It should be mentioned that the Spruce sample used here was prepared by our colleague Dr. Maria Adobes-Vidal in the institute of Wood Materials Science, ETH, Zurich, and our group performed further AFM measurements on this sample.

\section{Microfibrils structure in the S2/S3 region}

Figure 3.16 shows the cell wall's radial section, where the S2 and S3 layers are visible. At the upper part of Figure 3.16, various parallel lines from the S2 layer toward the lumen are apparent, which presumably refer to the S3 layer. This layer is the last fibrillar layer at the cell wall's inner boundary, with a thickness of about 100-200 nm.

Figure 3.17 shows the cross-sectional view of the wood cell wall near the lumen region. A borderline shows a dark layer in the most inner part of the S2 layer, which is presumably related to the S3 layer. The microfibrils are almost uniform in the S2 layer, where they are more extensive and randomly arranged in the area close to the S3 layer. This could be related to the change of the microfibril angle in the transition zone between the S2 and S3 layers. 


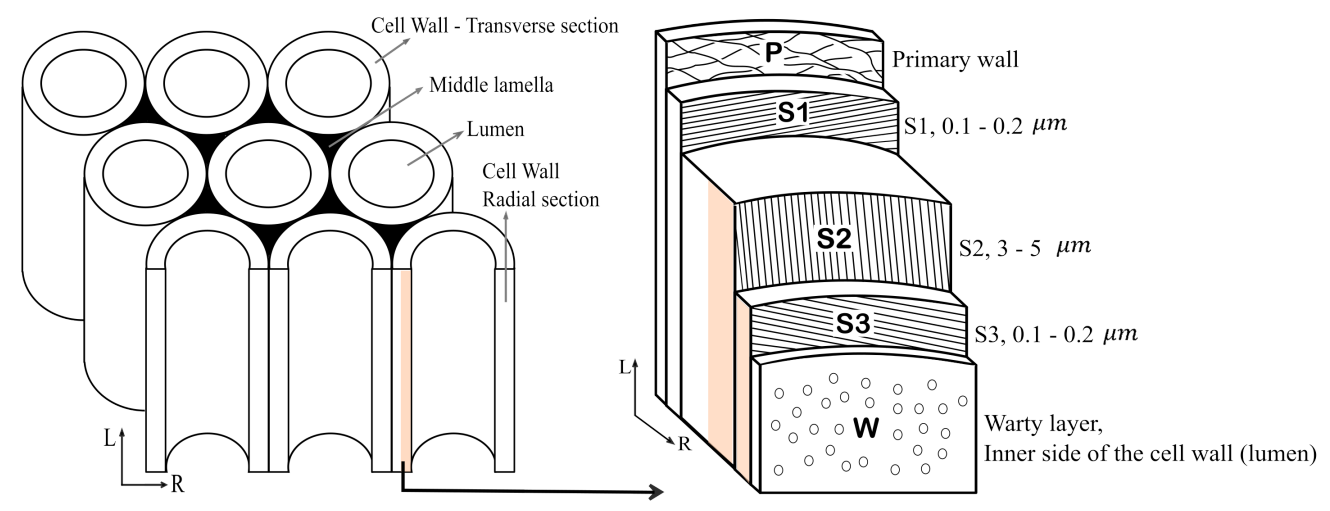

(a)

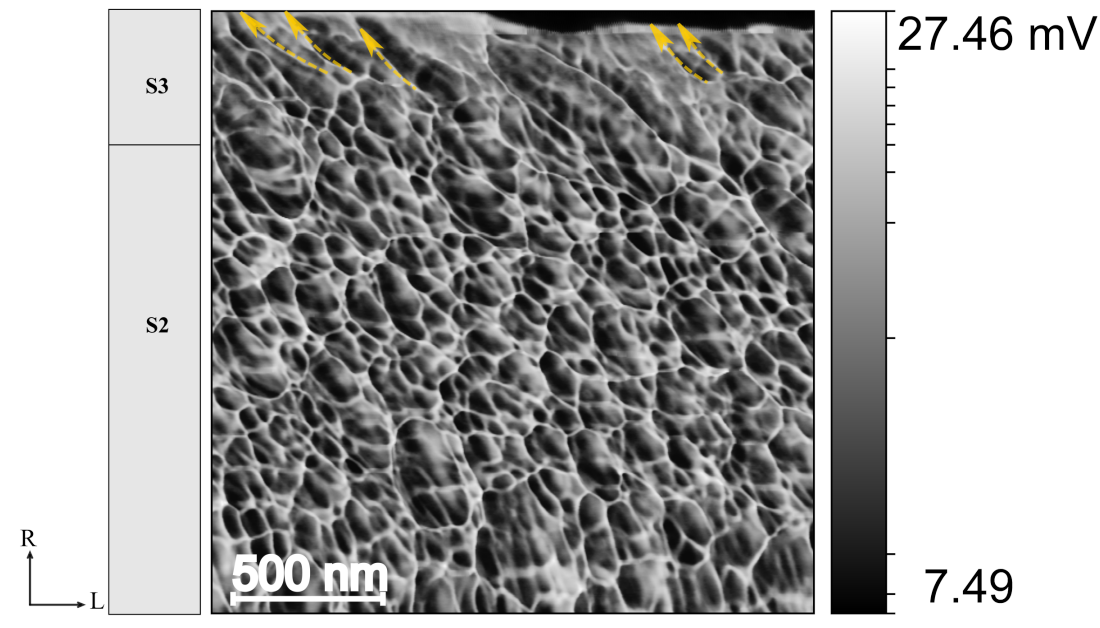

(b)

Figure 3.16: (a) Schematic illustration of the radial section, the highlighted zone shows the corresponding scanned area with atomic force microscopy. (b) Dissipation map of the S2/S3 layers. 


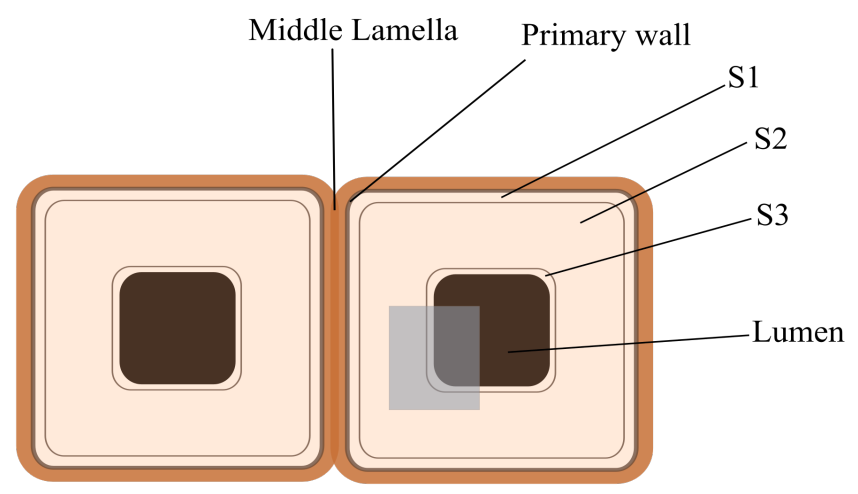

Transverse section
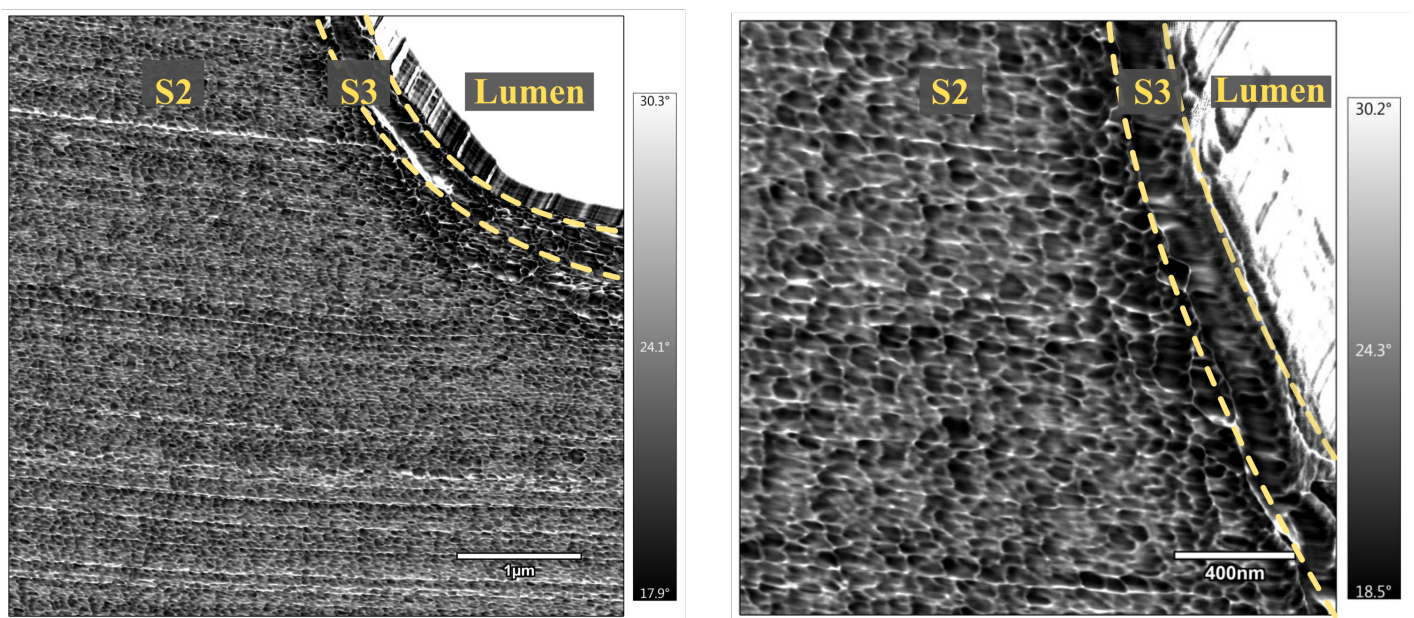

Figure 3.17: The AFM image of the pinewood cell wall's cross-section in two different magnifications. The differences between the S2 and S3 layers are visible. The highlighted area in the schematic picture refers to the scanned area with atomic force microscopy.

\section{Adjacent cell walls}

The two adjacent cell wall morphology is another important characteristic in the wood cell wall, shown in Figure 3.18. The cell walls are labeled as cell wall (1) and cell wall (2) in Figure 3.18(b,c). The microfibrils are in an almost cross-sectional view in the cell wall (2); however, they appear in a diagonal view in the cell wall (1).

Certainly, this is because of the helical arrangement of the cellulose fibers. Heyn et al. [211] already observed these topographic features with transmission electron microscopy. According to their observation [211], this morphological representation might be related to 


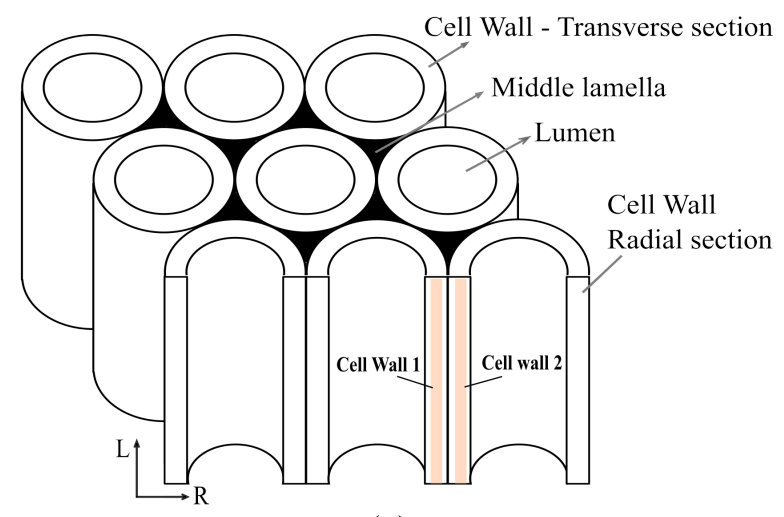

(a)
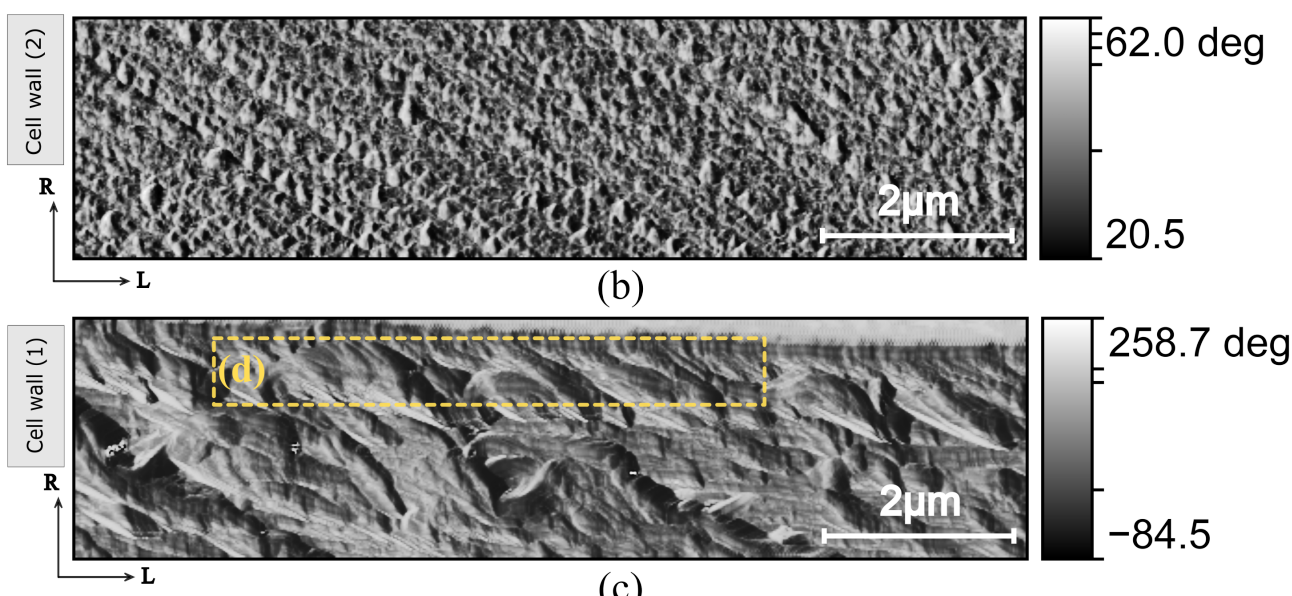

(c)
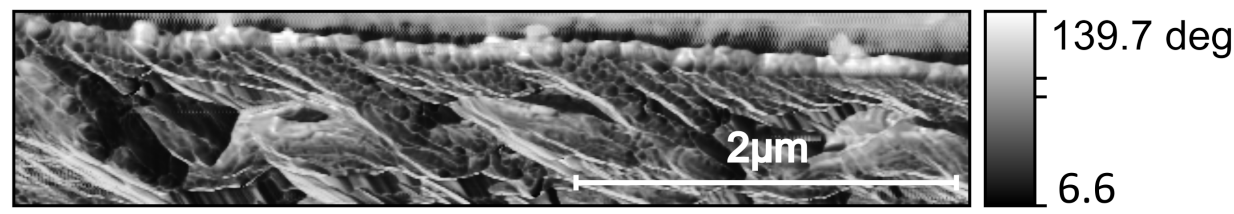

(d)

Figure 3.18: (a) Schematic illustration of the wood cell wall's radial view and the highlighted scanned area, (b,c) Phase map of the adjacent cell walls, (d) The higher magnification of the highlighted area in figure c. The radial direction of fibers in the S1 layer is visible.

the plane of sectioning. As shown in Figure 3.19, the sectioning plane could form a different angle with the fibrillar helix during the sample preparation. If the plane of sectioning forms the same angle by the helix, this plane would be parallel to the helix at one side of the lumen and perpendicular to the helix at the other side of the lumen. 
Further, the microfibrils' radial orientation in the S1 layer of the cell wall (1) is clearly visible in Figure 3.18(d), where the cellulose microfibrils spin in the right-hand direction of the cell axis.

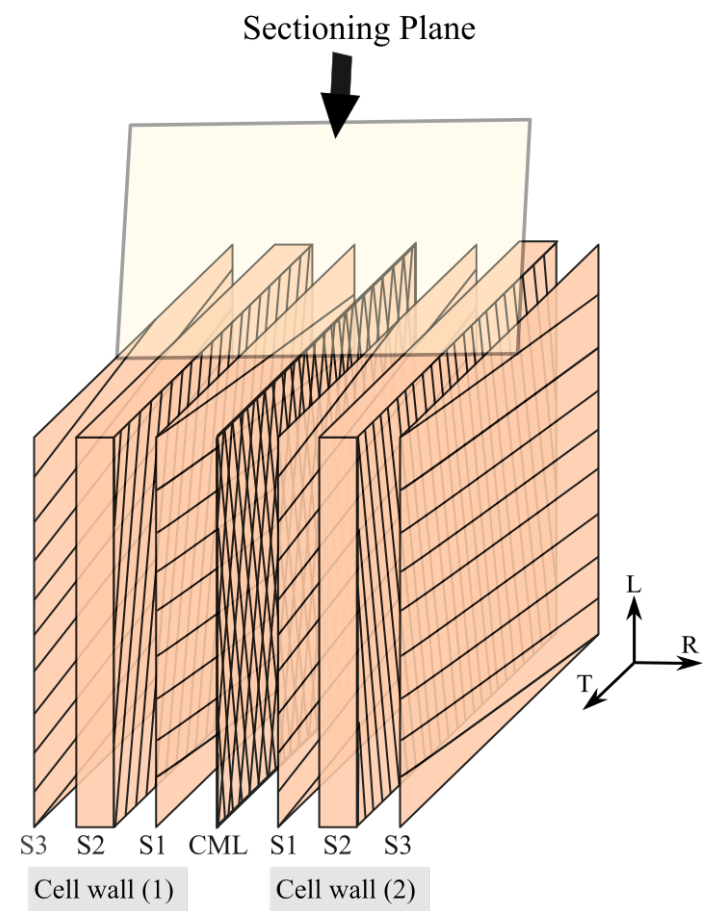

Figure 3.19: Schematic illustration regarding the sectioning plane and the fibrillar helix angle for two adjacent cell walls [137].

\section{Middle lamella and lignin distribution}

Figure 3.20 shows the cross-section of two adjacent wood cell walls. The middle lamella and adjacent primary walls are so-called compound middle lamella (CML), with a thickness of approximately $300 \mathrm{~nm}$. A globular structure in the S1 layer and middle lamella regions is visible. This morphological structure also exists in the S2 layer but on a much finer scale. It should be mentioned that the Spruce sample used for this image is the same as the sample presented in Figure 3.15, which was prepared in the institute of Wood Materials Science, ETH university. And the author performed further AFM measurements on this sample at the University of Goettingen.

By knowing the fact that in the middle lamella, the cellulose concentration is very low, 


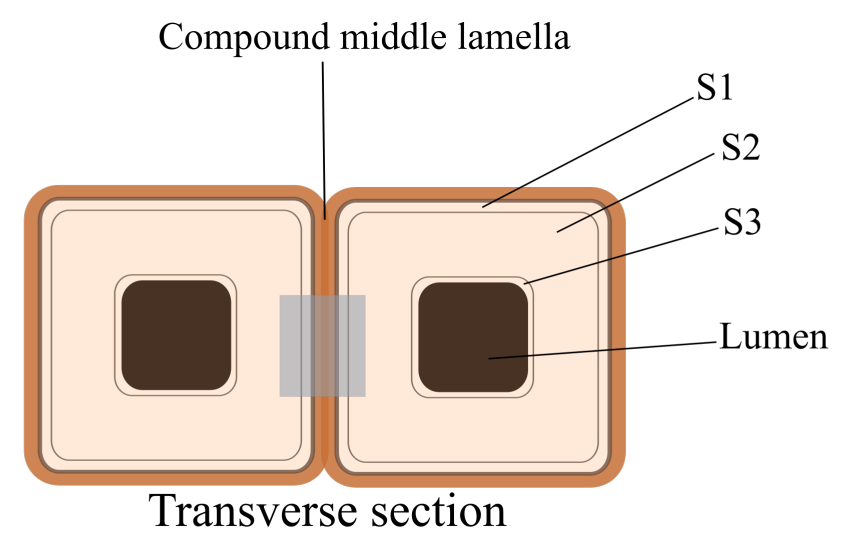

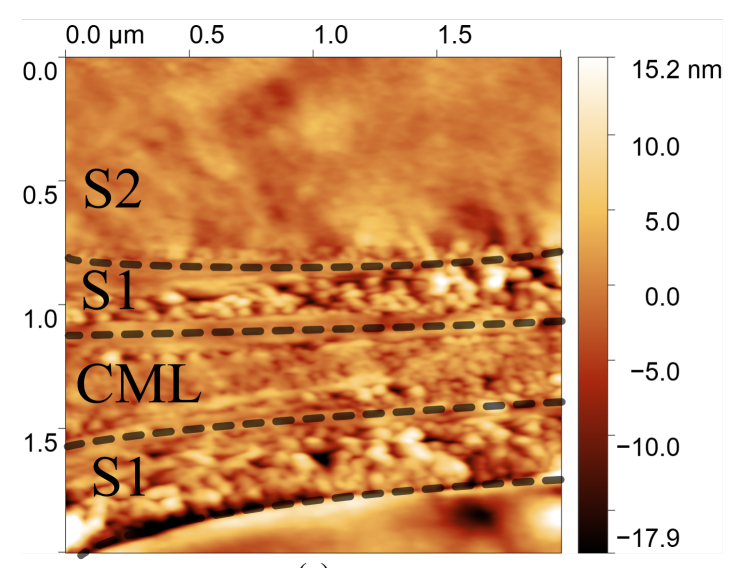

(a)

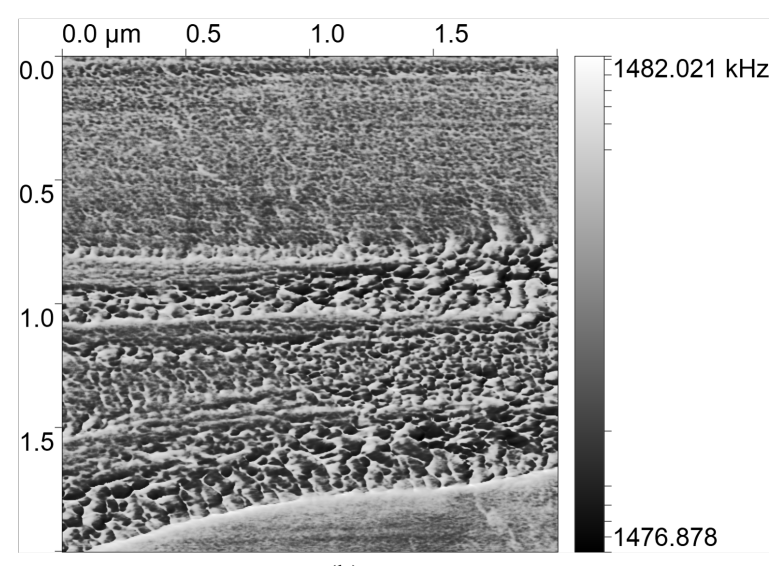

(b)

Figure 3.20: The AFM images of two adjacent cell walls shows the middle lamella and secondary layers. (a) topography and (b) frequency map. The corresponding scanned area is highlighted in the schematic picture of the wood cell wall's transverse section.

existing the granular aggregates in the middle lamella in Figure 3.20 presumably suggests that lignin in the wood cell wall is the component with the potential to form aggregates. This observation agrees with Donaldson et al. [177], which observed lignifying cell wall of pine wood by field emission electron microscopy and suggesting that lignin has a tendency to form aggregates. The globular aggregates of lignin in the wood cell wall are also reported in other studies. Terashima et al. [144] reported bead-like inclusions with the size of $35 \pm$ $5 \mathrm{~nm}$ in the middle lamella and cell corner regions distributed randomly.

Figure 3.21 shows the field-emission-scanning-electron microscopy (FE-SEM) image of the CML and the S1 layers in the transverse section of the spruce cell wall, using a backscattered electron detector (BSE). This figure is presented from the work of Fromm et 
al. [138]. The bright microfibrils and the dark lignin regions are shown, where lignin is visible as globular aggregates in CML and S1 layers.

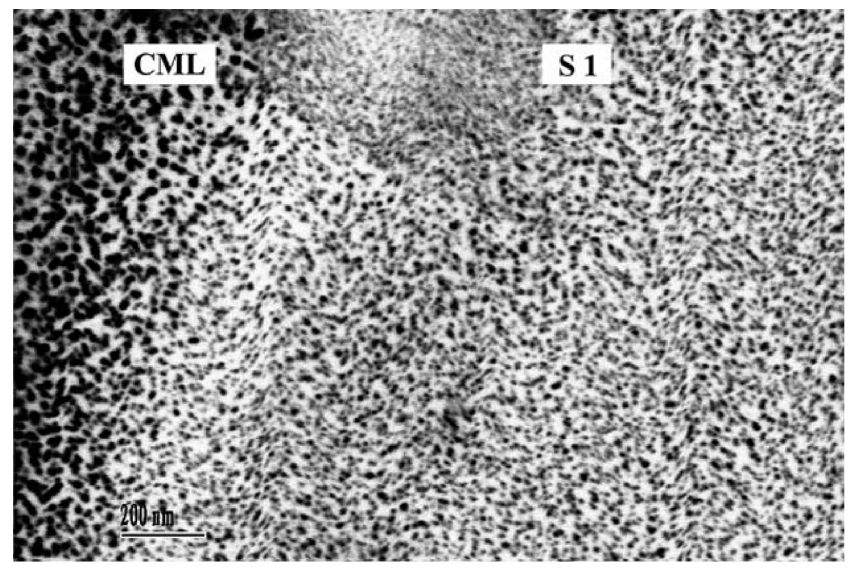

Figure 3.21: FE-SEM micrograph of spruce tracheid taken with a BSE detector. The transverse section shows lamellae of bright cellulose microfibrils and dark lignin aggregates in the S1 and compound middle lamella (CML) layers. Reprinted from Fromm et al. [138], Copyright 2003, with permission from Elsevier.

\section{Microfibrils structure in the lumen}

So far, while much attention has been focused on the secondary layers, it was not much concerned with examining the warty layer and lumen area with atomic force microscopy. This layer, however, seems to be interesting regarding the fibril arrangement. Figure 3.22 shows the wood cell wall's inner side (the surface lumen), scanned with atomic force microscopy.

Knowing that the crystalline silicon tip height is about $14 \mu \mathrm{m}$, it was possible to examine the lumen gently using the AM-FM method. It should be mentioned that this observation has been made on the natural wood cell wall, without any treatment and any knife cut in the lumen surface. So there is no damage to this layer. As shown in Figure 3.22, the parallel oriented microfibrils (with a beaded string appearance) wounded inside the lumen are visible, with an angle of approximately $45^{\circ}$ regarding the cell axis. The twisting or the criss-cross of elementary fibrils is also apparent (Figure 3.22(c)).

The lumen surface is the so-called warty layer in literature because it consists of spherical 

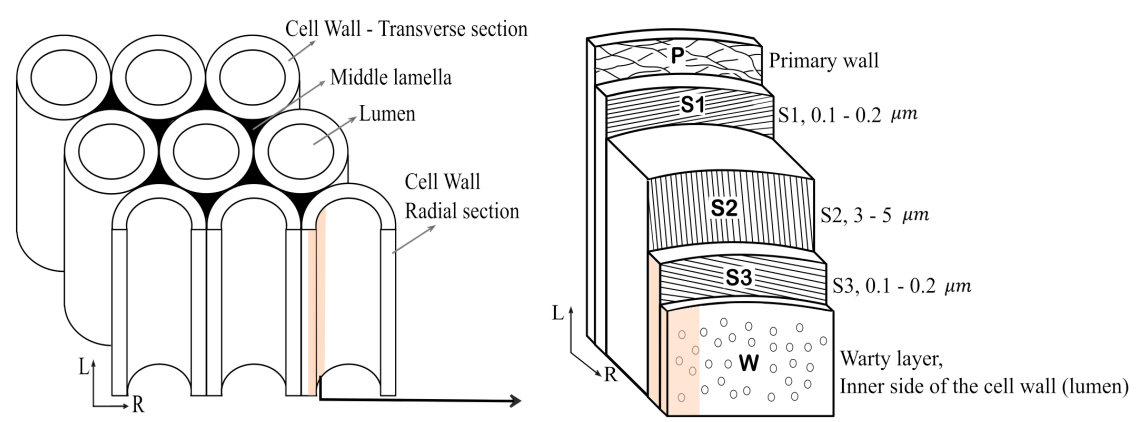

(a)

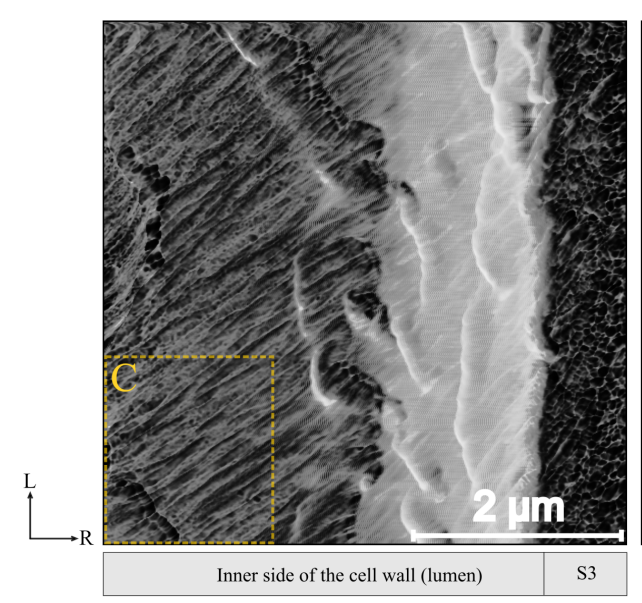

(b)

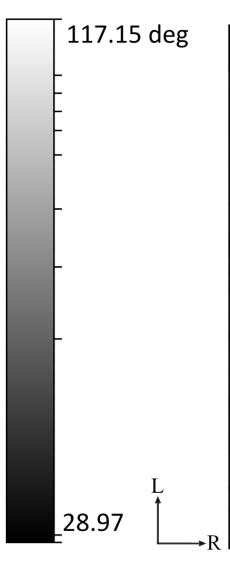

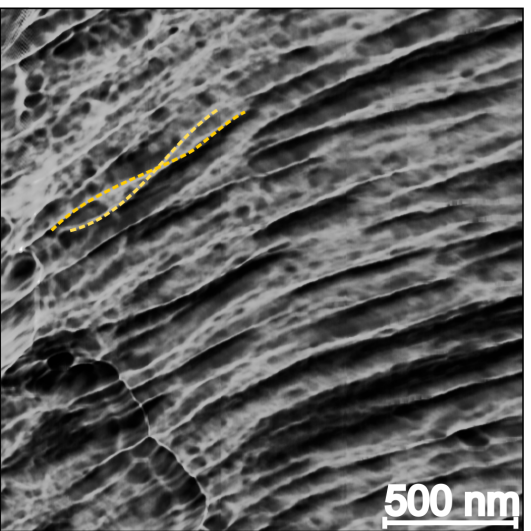

Inner side of the cell wall (lumen)

(c)

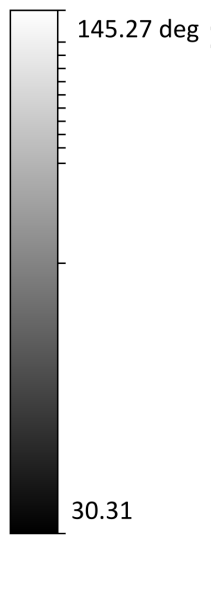

Figure 3.22: (a) Schematic picture of the radial section and secondary layers of the wood cell wall. The scanned area with atomic force microscopy is highlighted. (b) Phase map of inside the lumen from the radial section shows the microfibrils' helical orientation, (c) Higher magnification of the labeled area in Figure (b). The twisting of the fibers is observable.

particles (warts). The warty layer's appearance can vary concerning the number and size of warts and the presence or absence of the extra lining layer [212].

In Figure 3.22, the thickening of the S3 microfibrils and the end of microfibrils' bundles are what can be seen as warts of the lumen surface. It seems that the lining layer is not dense enough to embed the S3 microfibrils' orientation within the lumen. This is in agreement with the work done by Kuo et al. [213]. They found that the lumen surface of red pine tracheids was relatively free of encrustation, allowing them to see the S3 layer's microfibrillar orientation [213]. It is shown that warts' distribution pattern closely followed 


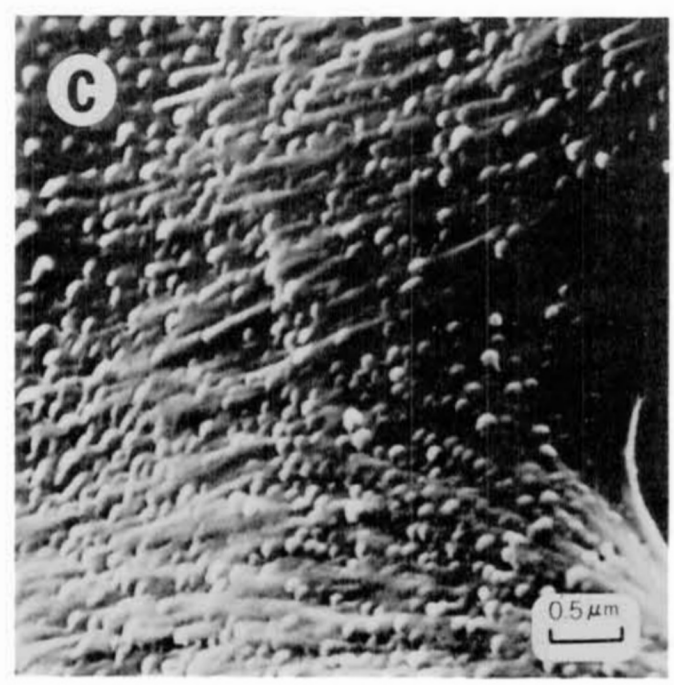

Figure 3.23: SEM image of the warty layer from the water-extracted heartwood tracheids [213]. The warts are located at the end of the microfibril bundles.

the path of the S3 layer's microfibrils. As shown in the SEM micrograph (Figure 3.23), many warts are located at the end of the bundles of S3 microfibrils. The possible mechanism of the S3 layer and warty layer relationship was clarified by Kuo et al. [213]. Microfibrils in the S3 layer accumulate in an aggregate shape in the final step of cell wall formation. In addition, the cells stop producing microfibrils at the end of microfibrillar bundles and deposit a terminating mixture of lignin and hemicellulose. In the AFM image presented in Figure 3.22, the end of the microfibrillar bundles are visible as dark circular areas, which is related to the lignin hemicellulose termination.

\subsection{Conclusion}

In the presented study, we used Atomic Force microscopy to obtain detailed information about the wood cell wall's ultrastructural arrangement. The AFM measurements on the wood cell wall were performed with the AM-FM method and Hertzian contact model. In this method, the second eigenmode could explore the dissipative and conservative interactions between the tip and sample. This work demonstrated that the AM-FM method is a fast, high-resolution technique that can represent the wood cell wall's nanomechanical 
mapping.

Evaluating the distribution of modulus values in relation to different surface curvature values revealed that the contrast in the elastic modulus map is primarily due to variance in material stiffness rather than variation in surface curvature. However, the close correlation between topography and elastic properties strongly suggests that the topography is created by the interaction of the cutting edge with the local material properties of the wood cell wall.

The obtained Young's modulus and phase lag maps of the wood cell wall exposed a structure composed of ca. $30 \mathrm{~nm}$ diameter inclusions with a low storage modulus embedded in a matrix with a high storage modulus. This result reveals that the ultrastructural arrangement of the wood cell wall is presumably composed of lignin inclusions embedded in a cellulose matrix which is in contrast with what has been taken for granted in years and is still an open and fascinating question!

The different morphology of the S1, S2, and S3 layers of the wood cell wall in radial and transverse sections were investigated with atomic force microscopy. The combined crosssectional and radial sectional views of the cell wall could provide a better understanding of the 3D assembly of the wood cell wall in nanoscale.

A globular structure was identified in the middle lamella, which has a higher lignin concentration. This structure was also found in the secondary layers of radial and transverse sections but in a more delicate form. The presence of the granular aggregates in the middle lamella indicated that lignin is the component with the potential of forming aggregates in the wood cell wall, at least as a result of interactions with the cutting blade.

The rounded or ellipsoidal appearance of the globular structure was significantly affected by the direction of the sectioning plane in relation to the fibers' orientation in the helical structure, whether the sectioning plane was precisely perpendicular or more inclined to the microfibrils' axis.

According to the AFM picture of the inner side of the cell wall lumen, the distribution pattern of warts followed the direction of the S3 layer's microfibrils. Moreover, the end of the microfibril bundles was visible as dark circular areas, which could be related to lignin 
and hemicellulose deposition. 
1103 ULTRASTRUCTURAL ARRANGEMENT OF THE WOOD CELL WALL IN NANOSCALE 


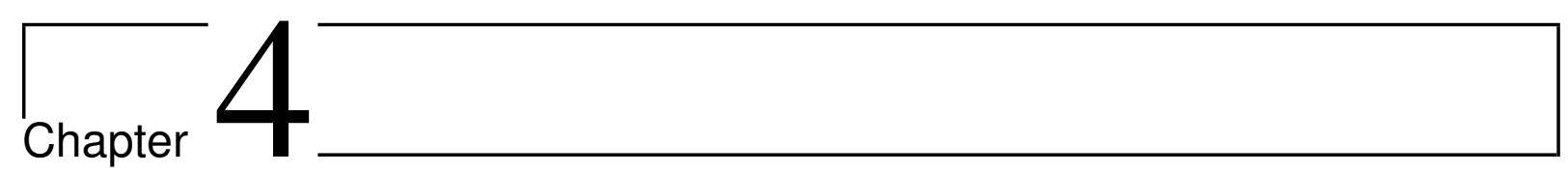

\section{Summary}

Wood, as one of the most renewable sources in nature, is currently exceeding the scope of its traditional use. The smart nature of wood and its functional properties for bearing mechanical loads attract researchers' interest to learn from nature and apply it for developing new materials in nanotechnology. Given the importance of cell wall microstructure and nanostructure in the mechanical properties of wood, the main challenge is to discover these properties at the micrometer and nanometer scales.

This thesis aimed to introduce, develop and apply micrometer and nanometer scale analysis methods in wood science, using nanoindentation and Atomic Force Microscopy. These techniques can be used for detecting wood cell wall mechanical properties and observing the fibrillary arrangement of the wood cell wall in different secondary layers.

The nanoindentation measurements revealed the local cell wall characteristics, including hardness, storage modulus, loss modulus, and loss factor in microscale. It was shown that where cellulose fibers and lignin are designed to increase cell wall stiffness and strength, the interface between cellulose fibers and matrix plays an essential role in transmitting stresses and dissipating energy. Assuming that the cellulose fibers' radius remains constant, increasing the volume fraction of cellulose results in an increase in the interface between fibers and matrix, resulting in a simultaneous increase in the storage modulus and loss modulus of the cell wall. 
According to the thermal treatments applied on pine as softwood and eucalyptus as hardwood, a moderate improvement of elastic modulus and hardness was observed in pinewood. However, the thermal treatment could not significantly affect the hardness and elastic modulus of eucalyptus wood. Despite that, a considerable reduction in the loss factor was observed for both pine and eucalyptus wood after thermal treatment. Regarding this fact, the function of hemicelluloses is suggested to be very important for the cell wall's overall damping efficiency.

In this study, the AM-FM method in Atomic Force Microscopy showed a fast, highresolution approach for providing the wood cell wall's nanomechanical mapping. The different layers of the wood cell walls in the radial and transverse sections were examined with atomic force microscopy. The combined cross-section and radial sectional view could provide a better understanding of the 3D assembly of the wood cell wall in nanoscale.

According to AFM maps, a globular structure was identified in the middle lamella. This structure was also found in a more delicate shape in secondary layers of the radial and transverse sections. The rounded or ellipsoid appearance of the globular structure was significantly influenced by the sectioning plane's direction concerning the fibers' orientation.

\section{Suggestions for future research:}

The AFM method described in this study could be further developed to observe treated wood cell walls and obtain more information regarding the cellulose and lignin distribution at the molecular scale.

The findings of the current study suggest that the cell wall's ultrastructure is composed of lignin inclusions in a cellulose matrix, which contradicts long-held assumptions and remains an interesting topic for future research. In this case, It is essential to note the association of the cutting edge with the local material properties of the wood cell wall in creating the topography.

The wood cell wall models must be reconsidered with the theoretical approaches for explaining the wood cell wall's exclusive characteristics such as damping, shrinkage, and flexural rigidity. It is necessary to discuss different models of the wood cell wall's nanostructural arrangement not only from the anatomical point of view but also concerning the optimized mechanical functions of the tree as a natural material. 


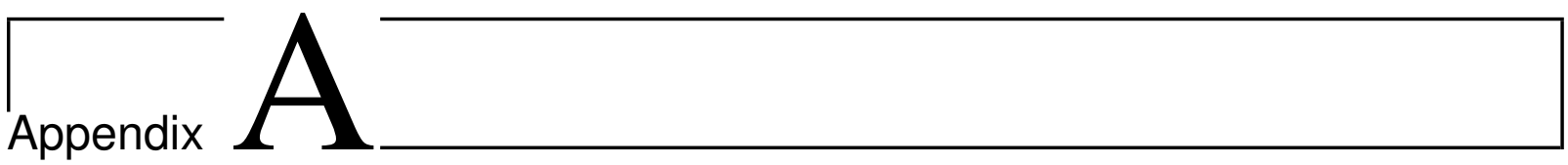

\section{Composite model for cell wall including interfaces}

In general, the elastic modulus of a composite could be predicted from the mechanical properties and volume fraction of its components. We assumed that the wood cell wall material is a three-phase composite composed of cellulose fibers, lignin matrix, and the interfacial layer between cellulose fiber and lignin matrix. So that the interface contribution is examined independently from those of the fiber and the matrix. The relevant assumed composite body is represented in Figure A.1. Since the fiber-reinforced composite is a periodic structure, the specific unit cell of this periodicity is illustrated in Figure A.1(c).

Based on this assumption, the upper-bound elastic modulus of the wood cell wall composite with aligned cellulose fibers in the lignin matrix is calculated via the rule of mixtures by assuming a uniform applied strain in the system (Voigt model) for a 3 phase composite. Where $V_{C}$ is the volume of the cellulose, $V_{L}$ is the volume of lignin, and $V_{\text {Int }}$ is the volume of the cellulose-lignin interface. And $f_{c}, f_{L}$, and $f_{\text {Int }}$. are the volume fractions of cellulose, lignin, and interface, respectively:

$$
f_{c}+f_{L}+f_{\text {Int }}=1
$$




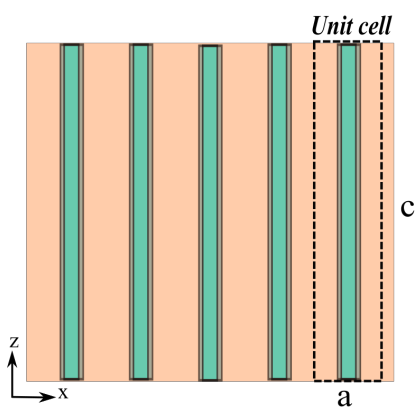

(a)

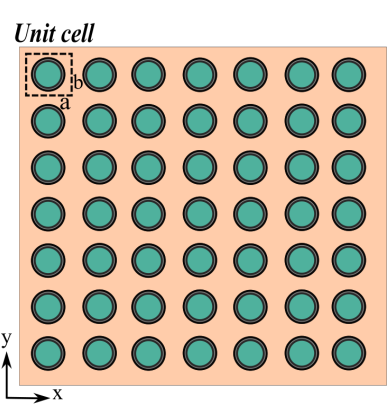

(b)

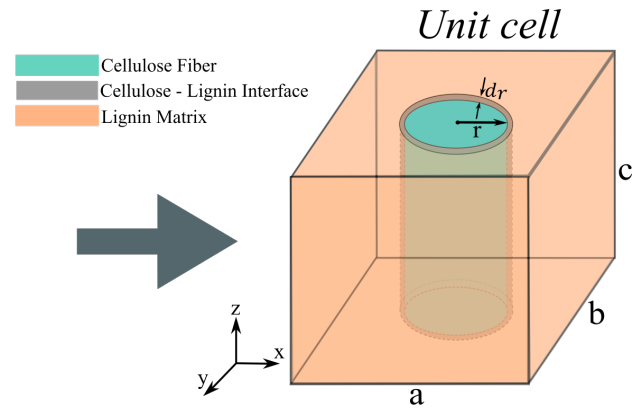

(c)

Figure A.1: Unidirectional fiber-reinforced composite with the isotropic matrix in (a) longitudinal and (b) transverse plane. The dashed lines in Figure (a) and (b) represent the unit cell in the periodic structure of the composite (c) Three-dimensional view of the unit cell in the composite structure.

$$
\begin{aligned}
& f_{c}=\frac{V_{C}}{\left(V_{C}+V_{L}+V_{\text {Int }}\right)}=\frac{\pi r^{2}}{a b} \\
& f_{\text {Int }}=\frac{V_{\text {Int }}}{\left(V_{C}+V_{L}+V_{\text {Int }}\right)}=\frac{2 \pi r d_{r}}{a b} \\
& f_{L}=\frac{V_{L}}{\left(V_{C}+V_{L}+V_{\text {Int }}\right)}=\left(1-f_{c}-f_{\text {Int }}\right)
\end{aligned}
$$

If we consider a force applied in a direction parallel to the fibers' long axes, each component's strain equals the composite's total strain.

$$
\varepsilon=\varepsilon_{c}=\varepsilon_{L}=\varepsilon_{\text {Int }}
$$

As stress is force per unit area, the overall stress can be calculated in the system as follows:

$$
\sigma=f_{c} \sigma_{c}+f_{L} \sigma_{L}+f_{\text {Int }} \sigma_{\text {Int }}
$$


Combining equations A.5 and A.6 gives an expression for the axial Modulus. So, based on the rule of mixtures, the elastic modulus of the composite parallel to the fibers can be estimated as follows:

$$
E=f_{c} E_{c}+f_{L} E_{L}+f_{\text {Int }} E_{\text {Int }}
$$

where $E_{c}$ is the elastic modulus of the cellulose fiber, $E_{L}$ is the lignin's elastic modulus and $E_{I n t}$ is the elastic modulus of the interface.

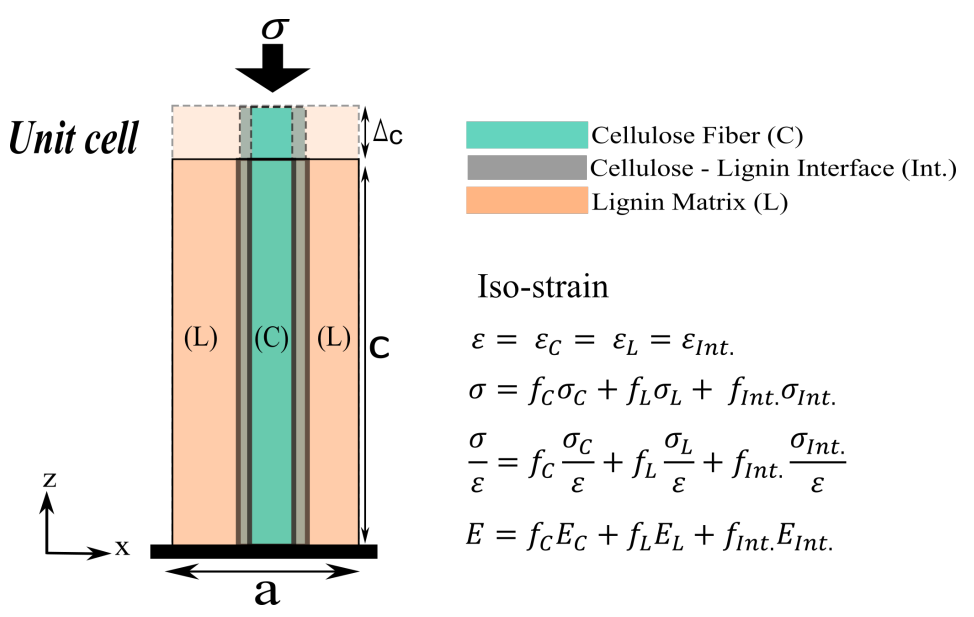

Figure A.2: The Voigt model or Iso-strain model providing the upper bound of the overall elastic modulus of the composite when the stress is applied along the fiber direction.

Consequently, the application of the rule of mixtures can be used for the evaluation of the complex modulus $\left(E^{*}\right)$ in the composite as below:

$$
E^{*}=f_{c} E_{c}^{*}+f_{L} E_{L}^{*}+f_{\text {Int }} E_{\text {Int }}^{*}
$$

By knowing the fact that the complex modulus consists of two components, the storage and the loss moduli (equation 2.4); the average longitudinal storage modulus $\left(E^{\prime}\right)$ and loss modulus $\left(E^{\prime \prime}\right)$ of the wood cell wall as a fiber-reinforced polymer composite can be obtained as below:

$$
E_{\text {Cell-wall }}^{\prime}=\left[f_{c} E_{c}^{\prime}+f_{L} E_{L}^{\prime}\right]+\frac{2 \pi r d_{r}}{a b} E_{I n t}^{\prime}
$$




$$
E_{\text {Cell-wall }}^{\prime \prime}=\left[f_{c} E_{c}^{\prime \prime}+f_{L} E_{L}^{\prime \prime}\right]+\frac{2 \pi r d_{r}}{a b} E_{\text {Int }}^{\prime \prime}
$$

In the definition of the storage modulus, since the wood structure includes the very stiff cellulose fibers, the storage modulus of the interface is negligible, and the storage modulus of the cell wall could define as below:

$$
E_{\text {Cell-wall }}^{\prime}=\left[f_{c} E_{c}^{\prime}+f_{L} E_{L}^{\prime}\right]
$$

By assuming $d r \rightarrow 0$,

$$
\begin{aligned}
& f_{L}=\left(1-f_{c}\right) \\
& E_{\text {Cell-wall }}^{\prime}=\left[f_{c} E_{c}^{\prime}+\left(1-f_{c}\right) E_{L}^{\prime}\right]
\end{aligned}
$$

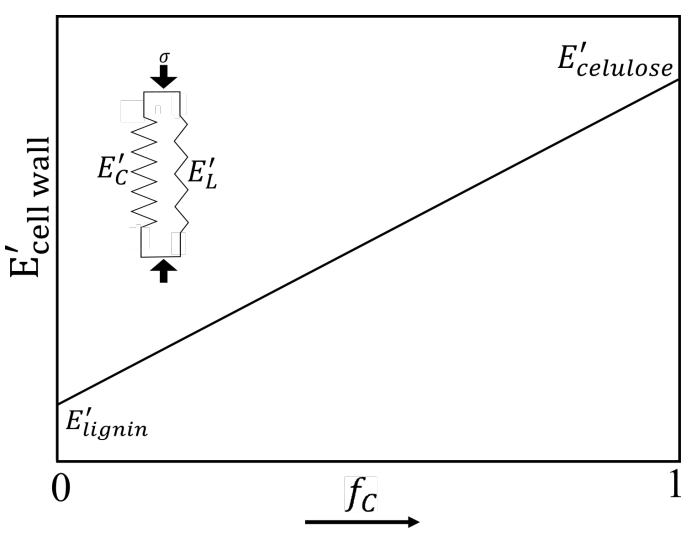

Figure A.3: The storage modulus of the cell wall as a function of cellulose volume fraction.

So, regarding equation A.13 and as shown in Figure A.3, the cell wall's storage modulus increases with increasing the volume fraction of the cellulose fibers. In this way, the next question that arises is how the storage modulus and loss modulus correlate with each other? and where increasing the cellulose fiber volume fraction concludes to the increase of storage modulus, which factor has the predominant effect on increasing the loss modulus?

The loss modulus highlights the viscous properties of polymeric-based materials and represents energy lost during one cyclic load. Although increasing the volume fraction of 
cellulose as a crystalline polymer with its high stiffness should not have a predominant effect on increasing the loss modulus, the interface between cellulose fibers and matrix should play a crucial role. Adams et al. [143] mentioned that the friction of fibers against the matrix and the shear stress affected by hydration and chemical forces are the main factors in dissipation energy in the composite materials. Regarding this fact on examing the loss modulus of the wood cell wall, the interface between polymeric components is much more essential than the viscoelastic behavior of each polymer itself. So the first term in equation A.10 is negligible, and the average loss modulus in the cell wall is predominated with friction processes in the interface of the fibers and matrix as below:

$$
E_{\text {Cell-wall }}^{\prime \prime}=\frac{2 \pi r d_{r}}{a b} E_{\text {Int }}^{\prime \prime}
$$

Where $\frac{2 \pi r d_{r}}{a b}$ is the volume fraction of the interfaces in the unit cell of the fiber composite structure. By assuming a constant dimension for the cellulose fibers ( $r=$ constant), increasing the volume fraction of the cellulose fibers and consequently increasing the periodicity in the structure concludes to the smaller dimensions $(a, b)$ of the unit cell (Figure A.4). The smaller unit cells in the system cause the larger volume fraction of the interfaces in the structure. This concludes with the higher shear stresses and friction in the structure, which causes more dissipation energy. This implies that where the wood cell wall is designed to resist tension/compression forces through a fiber composite performance; by which the matrix resists part of the tension/compression forces and the balance is taken by the fibers, the composite performance depends greatly on the mechanisms through which the stresses are transferred between the fiber and the matrix. Thus, the mechanical properties of the fiber-matrix interface that control such transfer process should significantly affect the overall structural response of the composite. In this way, by increasing the cell wall's storage modulus, the loss modulus will spontaneously increase. 


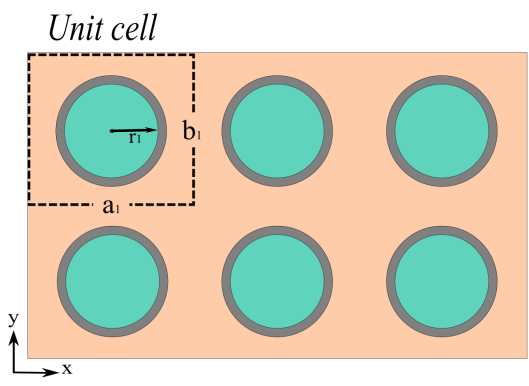

(a)

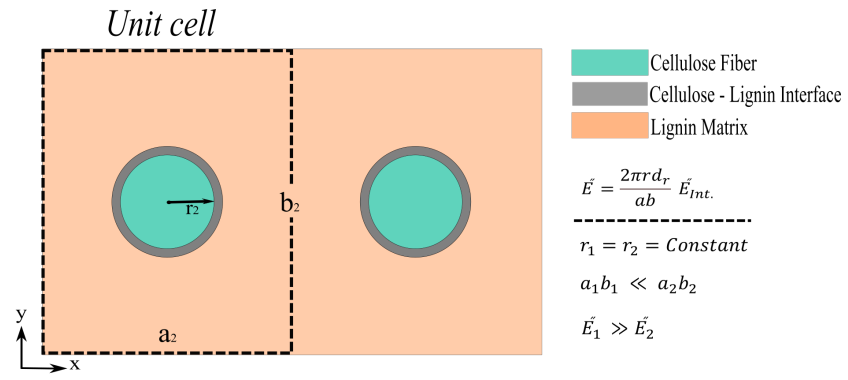

(b)

Figure A.4: A periodic structure that resembles the cross-section of cellulose fibers embedded in the matrix of lignin (a) structure1: higher volume fraction of the cellulose fibers (b) structure 2: lower volume fraction of the cellulose fibers. 


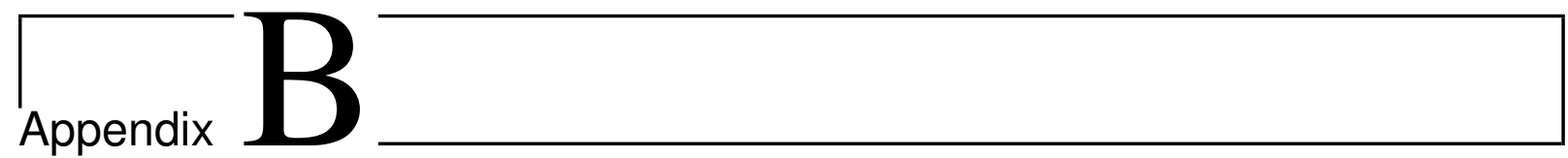

\section{Proposed model for nanostructural assembly of the wood cell wall}

This research suggests a new model for cell wall structure based on atomic force microscopy images, which remains an open question.

Our hypothesis introduces the cell wall as a honeycomb-like cellulose frame that acts as the main load-bearing element in the cell wall filled with lignin (Figure B.1, Model B). The AFM elastic modulus map is consistent with this model. Furthermore, it should keep in mind that according to the cell wall ontogeny, the polysaccharides deposition plays an essential role as a template before lignin macromolecule formation in the cell wall [214].

Model A

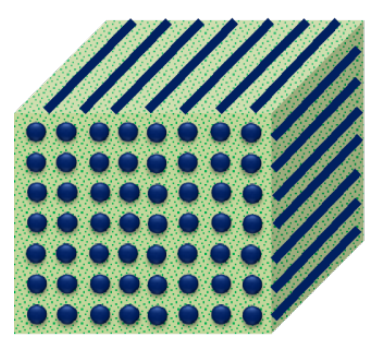

Model B

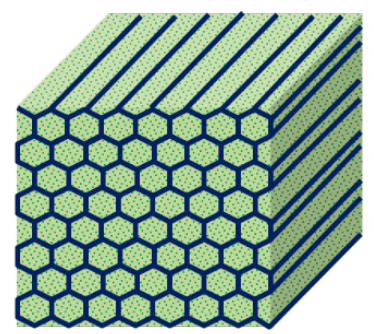

Figure B.1: (a) Unidirectional fiber composite model. (b) Honeycomb-like cellulose frame filled with lignin model.

By simplifying the long prismatic cellulose frame to a regular hexagon honeycomb, and 
assuming the wall thickness to be $t=3 \mathrm{~nm}$ and the side length of this hexagon to be $h=l=15$ $\mathrm{nm}$, the elastic modulus of the honeycomb cellulose frame was calculated in the axial and transverse directions:

$$
\begin{aligned}
& E_{\text {axial }}=\frac{t}{l} \frac{h / l+2}{2(h / l+\sin \theta) \cos \theta} \frac{2}{\sqrt{3}} \frac{3}{15}=130 \frac{2}{\sqrt{3}} \frac{3}{15}=30 \mathrm{GPa} \\
& E_{\text {transverse }}=\left(\frac{t}{l}\right)^{3} \frac{\cos \theta}{(h / l+\sin \theta) \sin ^{2} \theta} \frac{4}{\sqrt{3}}\left(\frac{3}{15}\right)^{2}=130 \frac{4}{\sqrt{3}}\left(\frac{3}{15}\right)^{2}=2.40 \mathrm{GPa}
\end{aligned}
$$

Another fundamental factor that could make the new proposed model more verifiable is the importance of the material's shape and structure on mechanical properties. It is well known that wood as a hybrid material with a high ratio of strength to weight is a combination of mainly three different building blocks (cellulose, lignin, and hemicellulose) in an essential relative volume fraction, which is optimally serving the mechanical resistance purpose. A tree is a slim stem with a height of more than 50 meters, bearing the large static and dynamic forces of gravity and wind-loads [8].

By assuming the same mass of cellulose for both cell wall models (model A: the old model, model B: the new proposed model), the moment of inertia and consequently the material's flexural rigidity (EI) of the cell wall for the new model is much more robust by a factor of $R / t$. (Figure B.2). As a simple explanation, the higher the material's elastic modulus and the higher the object's moment of inertia, the less the structure will deflect under a given load.

$$
\begin{aligned}
& \text { for same mass: } \rho \pi r^{2}=\rho 2 \pi R t, r=(2 R t)^{\left(\frac{1}{2}\right)} \\
& \text { for same mass }: \frac{I_{t}}{I_{s}}=\frac{\left(\pi R^{3} t\right)}{\left(\pi / 4(2 R t)^{2}\right)}=\frac{R}{t}
\end{aligned}
$$




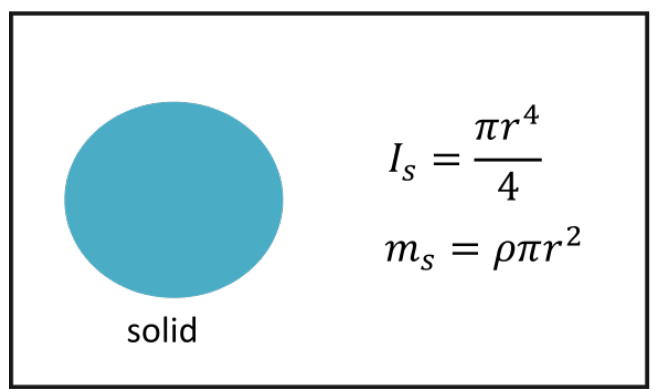

Model A

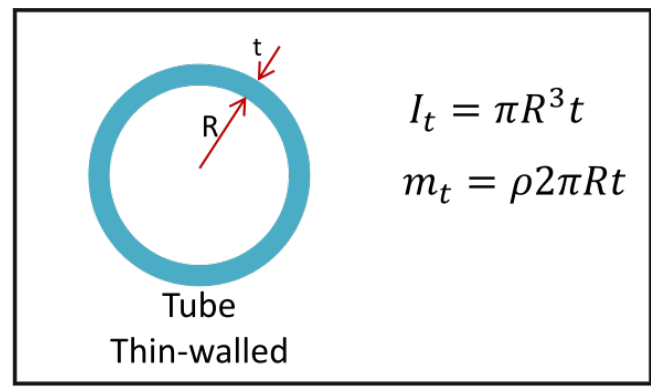

Model B

Figure B.2: Comparison of the moment of inertia for model A and model B.

This proposed structural model, however, is still open to further investigations. 
122 B PROPOSED MODEL FOR NANOSTRUCTURAL ASSEMBLY OF THE WOOD CELL WALL 


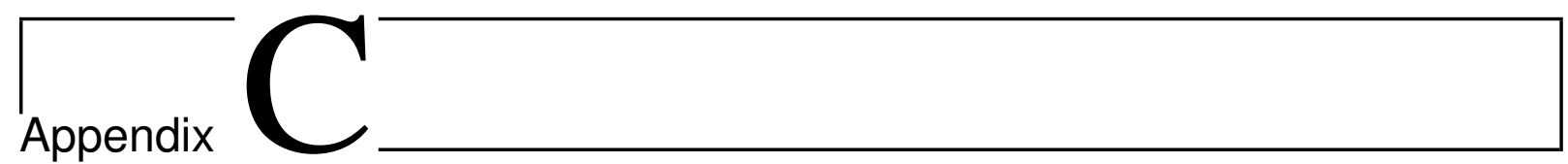

\section{Cell wall treatment}

\section{C.1 Delignification of the wood cell wall}

To better understand cellulose's role in the wood cell wall, we chose two different treatments with white-rot fungi attack and acidic bleaching delignification for degrading the lignin and maintaining the cellulose in the cell wall.

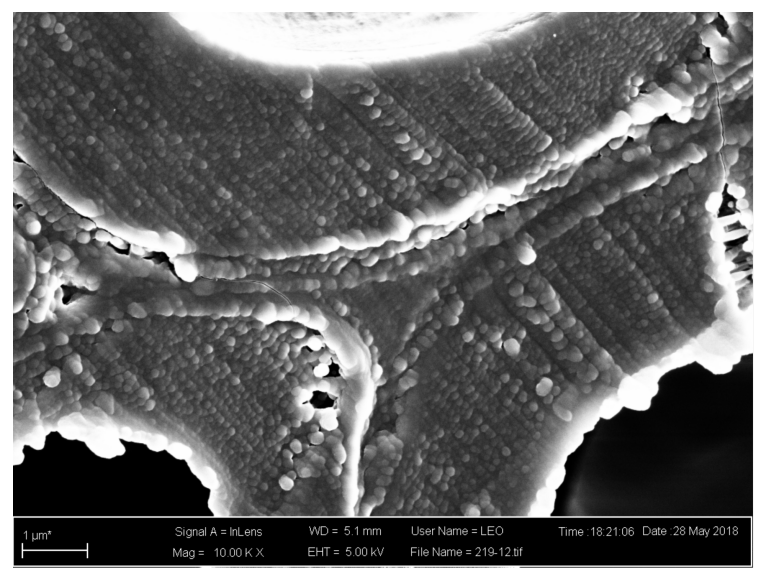

(a)

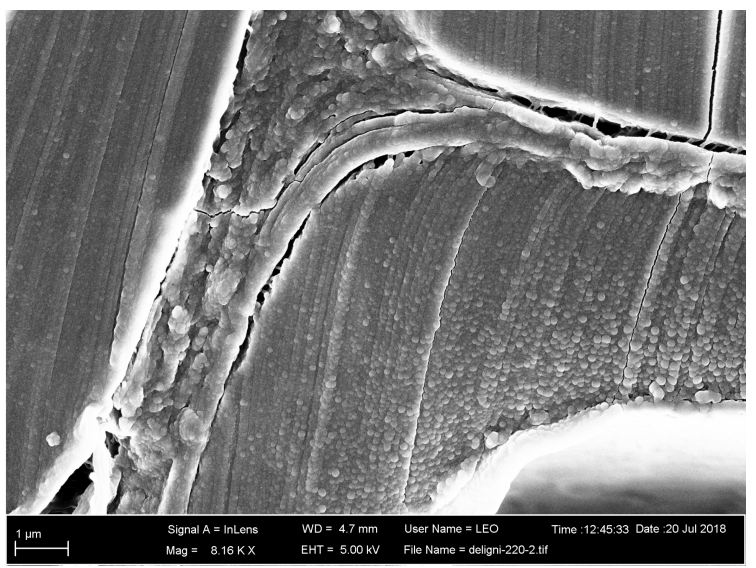

(b)

Figure C.1: Delignified wood cell wall with (a) white-rot fungi attack and (b) acidic bleaching procedure.

As shown in Figure C.1, after removing lignin from the cell wall structure, the structural 
pattern is not strongly influenced, and there is no severe interruption. The cell wall persists as a firmly coherent cellulose framework. This observation is in agreement with Bailey's report [18]. Bailey emphasized that even in walls that have been treated to extract their non-cellulosic components, the structure of tracheids and fibers are coherent. In fact, there are no discontinuities in the structural pattern after the removal of non-cellulosic components. The remaining cellulose exhibits a structural design similar to the natural wood cell wall, tightly woven together into a complex and firmly coherent structure.

\section{C.2 Cellulose degradation in the wood cell wall}

By using brown-rot fungi attack, the cellulose component was almost removed from the cell wall, and a long cord-like lignin structure in the cell wall was revealed (Figure C.2).

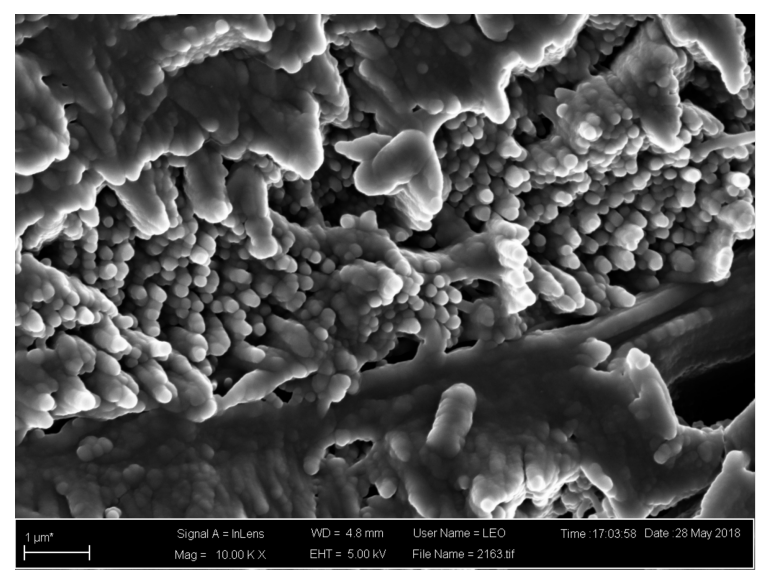

Figure C.2: Cellulose degradation in the cell wall with brown-rot fungi attack.

This observation is in agreement with the comments of Bailey [18]. It seems that after removing the cellulose from the cell wall, the lignin self-aggregates and forms a bead-like shape in a regular dimension.

The aggregation of lignin is observed in other works as well. Kang et al. reported that lignin self aggregates to form highly hydrophobic nanodomains [30]. Donaldson et al. [215] observed the lignified cell wall of pine by TEM and permanganate staining. They found that growing lignin aggregates in the middle lamella form roughly spherical structures 
within the randomly arranged matrix, while in the S2 layer, lignin forms greatly elongated structures, following the orientation of cellulose microfibrils.

Although hemicellulose's existence in the cell wall has an essential role as a coupling agent between cellulose chains and lignin macromolecules, the two most critical components targeted in our interpretation were cellulose and lignin. Hemicellulose tracing was skipped in our observations because the amount of hemicelluloses relative to cellulose and lignin concentration is small to affect the understanding of the cell wall's microstructure. 


\section{Bibliography}

[1] E. Wheeler. Wood: Macroscopic anatomy. In Encyclopedia of Materials: Science and Technology, pages 9653-9657. Elsevier, Oxford, 2001. ISBN 978-0-08-0431529. doi: https://doi.org/10.1016/B0-08-043152-6/01749-6. URL https://www . sciencedirect.com/science/article/pii/B0080431526017496.

[2] Terry Conners. Distinguishing softwoods from hardwoods. Agriculture and Natural Resources Publications, 105, 2015. URL https://uknowledge.uky.edu/anr_ reports/105.

[3] Sebastian Pfautsch, Teemu Hölttä, and Maurizio Mencuccini. Hydraulic functioning of tree stems-fusing ray anatomy, radial transfer and capacitance. Tree Physiology, 35(7):706-722, 07 2015. ISSN 0829-318X. doi: 10.1093/treephys/tpv058. URL https://doi.org/10.1093/treephys/tpv058.

[4] Lorna J. Gibson. The hierarchical structure and mechanics of plant materials. Journal of The Royal Society Interface, 9(76):2749-2766, 2012. doi: 10.1098/rsif. 2012.0341. URL https://royalsocietypublishing.org/doi/abs/10.1098/rsif. 2012.0341.

[5] David Cown. Variation in tracheid dimensions in the stem of a 26 year old radiata pine tree. Appita Journal, 28:237 245, 011975.

[6] J. Fahlén and L. Salmén. Cross-sectional structure of the secondary wall of wood fibers as affected by processing. Journal of Materials Science, 38(1):119- 
126, 2003. doi: 10.1023/A:1021174118468. URL https://doi.org/10.1023/A: 1021174118468.

[7] Alex Wiedenhoeft and Regis Miller. Structure and function of wood. Handbook of Wood Chemistry and Wood Composites, pages 9-33, 012005.

[8] R. E. Booker and J. Sell. The nanostructure of the cell wall of softwoods and its functions in a living tree. Holz als Roh- und Werkstoff, 56(1):1-8, 1998. doi: 10. 1007/s001070050255. URL https://doi.org/10.1007/s001070050255.

[9] Dietrich Fengel. The ultrastructure of cellulose from wood. Wood Science and Technology, 3(3):203-217, 1969. doi: 10.1007/BF00367212. URL https://doi. org/10.1007/BF00367212.

[10] D. Fengel. Ideas on the ultrastructural organization of the cell wall components. Journal of Polymer Science Part C: Polymer Symposia, 36(1):383-392, 1971. doi: https://doi.org/10.1002/polc.5070360127. URL https://onlinelibrary.wiley. com/doi/abs/10.1002/polc.5070360127.

[11] A. Frey-Wyssling. The fine structure of cellulose microfibrils. Science, 119(3081): 80-82, 1954. ISSN 0036-8075. doi: 10.1126/science.119.3081.80. URL https:// science.sciencemag. org/content/119/3081/80.

[12] J R Barnett and Victoria A Bonham. Cellulose microfibril angle in the cell wall of wood fibres. Biological reviews of the Cambridge Philosophical Society, 79(2): 461-472, May 2004. doi: 10.1017/s1464793103006377.

[13] Uwe G. Hacke and John S. Sperry. Functional and ecological xylem anatomy. Perspectives in Plant Ecology, Evolution and Systematics, 4(2):97-115, 2001. ISSN 1433-8319. doi: https://doi.org/10.1078/1433-8319-00017. URL https://www . sciencedirect.com/science/article/pii/S1433831904700426.

[14] B. A. Meylan and B. G. Butterfield. Helical orientation of the microfibrils in tracheids, fibres and vessels. Wood Science and Technology, 12(3):219-222, 1978. doi: 10.1007/ BF00372867. URL https://doi .org/10.1007/BF00372867. 
[15] Lloyd Donaldson and Ping Xu. Microfibril orientation across the secondary cell wall of radiata pine tracheids. Trees, 19(6):644, 2005. doi: 10.1007/s00468-005-0428-1. URL https://doi .org/10.1007/s00468-005-0428-1.

[16] Lloyd A. Donaldson and Pieter Baas. Wood cell wall ultrastructure the key to understanding wood properties and behaviour. IAWA Journal, 40(4):645 - 672, 16 Nov. 2019. doi: https://doi.org/10.1163/22941932-40190258. URL https://brill. com/view/journals/iawa/40/4/article-p645_3.xml.

[17] Marjan Sedighi-Gilani, Homeira Sunderland, and Parviz Navi. Microfibril angle nonuniformities within normal and compression wood tracheids. Wood Science and Technology, 39(6):419-430, 2005. doi: 10.1007/s00226-005-0022-0. URL https: //doi.org/10.1007/s00226-005-0022-0.

[18] I. W. Bailey. Cell wall structure of higher plants. Industrial \& Engineering Chemistry, 30(1):40-47, 01 1938. doi: 10.1021/ie50337a009. URL https://doi.org/10.1021/ ie50337a009.

[19] A. Frey-Wyssling. The ultrastructure of wood. Wood Science and Technology, 2(2):73-83, 1968. doi: 10.1007/BF00394956. URL https://doi.org/10.1007/ BF00394956.

[20] Jerrold Winandy and Roger Rowell. The chemistry of wood strength. Handbook of Wood Chemistry and Wood Composites, Second Edition, 05 1984. doi: 10.1021/ ba-1984-0207.ch005.

[21] T. Nishino. 4 - natural fibre sources. In Caroline Baillie, editor, Green Composites, Woodhead Publishing Series in Composites Science and Engineering, pages 49-80. Woodhead Publishing, 2004. ISBN 978-1-85573-739-6. doi: https://doi.org/10.1016/ B978-1-85573-739-6.50007-5. URL https://www.sciencedirect.com/science/ article/pii/B9781855737396500075.

[22] David L. VanderHart and R. H. Atalla. Studies of microstructure in native celluloses using solid-state carbon-13 nmr. Macromolecules, 17(8):1465-1472, 08 1984. doi: 10.1021/ma00138a009. URL https://doi.org/10.1021/ma00138a009. 
[23] Junji Sugiyama, Roger Vuong, and Henri Chanzy. Electron diffraction study on the two crystalline phases occurring in native cellulose from an algal cell wall. Macromolecules, 24(14):4168-4175, 07 1991. doi: 10.1021/ma00014a033. URL https://doi.org/10.1021/ma00014a033.

[24] Masahisa Wada, Tetsuo Kondo, and Takeshi Okano. Thermally induced crystal transformation from cellulose i $\alpha$ to i $\beta$. Polymer Journal, 35(2):155-159, 2003. doi: 10.1295/polymj.35.155. URL https://doi .org/10.1295/polymj.35.155.

[25] Takashi Nishino, Kiyofumi Takano, and Katsuhiko Nakamae. Elastic modulus of the crystalline regions of cellulose polymorphs. Journal of Polymer Science Part B: Polymer Physics, 33(11):1647-1651, 1995. doi: https://doi.org/10.1002/polb.1995. 090331110. URL https://onlinelibrary.wiley.com/doi/abs/10.1002/polb. 1995.090331110.

[26] Kohji Tashiro and Masamichi Kobayashi. Theoretical evaluation of three-dimensional elastic constants of native and regenerated celluloses: role of hydrogen bonds. Polymer, 32(8):1516-1526, 1991. ISSN 0032-3861. doi: https://doi.org/10. 1016/0032-3861(91)90435-L. URL https://www.sciencedirect.com/science/ article/pii/003238619190435L.

[27] Roger Rowell. Handbook Of Wood Chemistry And Wood Composites. CRC Press, 02 2005. ISBN 9780849315886. doi: 10.1201/b12487.

[28] Erich Adler. Lignin chemistry-past, present and future. Wood Science and Technology, 11(3):169-218, 1977. doi: 10.1007/BF00365615. URL https://doi. org/10.1007/BF00365615.

[29] Ronald Hatfield and Wilfred Vermerris. Lignin formation in plants. the dilemma of linkage specificity. Plant Physiology, 126(4):1351-1357, 2001. ISSN 0032-0889. doi: 10.1104/pp.126.4.1351. URL http://www.plantphysiol.org/content/126/ $4 / 1351$.

[30] Xue Kang, Alex Kirui, Malitha C. Dickwella Widanage, Frederic Mentink-Vigier, Daniel J. Cosgrove, and Tuo Wang. Lignin-polysaccharide interactions in plant secondary cell walls revealed by solid-state nmr. Nature Communications, 10(1): 
347, 2019. doi: 10.1038/s41467-018-08252-0. URL https://doi.org/10.1038/ s41467-018-08252-0.

[31] M.T. Holtzapple. Hemicelluloses. In Benjamin Caballero, editor, Encyclopedia of Food Sciences and Nutrition (Second Edition), pages 3060-3071. Academic Press, Oxford, second edition edition, 2003. ISBN 978-0-12-227055-0. doi: https://doi.org/10. 1016/B0-12-227055-X/00589-7. URL https ://www . sciencedirect. com/science/ article/pii/B012227055X005897.

[32] B. F. Tjeerdsma, M. Boonstra, A. Pizzi, P. Tekely, and H. Militz. Characterisation of thermally modified wood: molecular reasons for wood performance improvement. Holz als Roh- und Werkstoff, 56(3):149-153, 1998. doi: 10.1007/s001070050287. URL https://doi .org/10.1007/s001070050287.

[33] Michiel J. Boonstra, Joris Van Acker, Bôke F. Tjeerdsma, and Edo V. Kegel. Strength properties of thermally modified softwoods and its relation to polymeric structural wood constituents. Annals of Forest Science, 64(7):679-690, 2007. doi: 10.1051/ forest:2007048. URL https://doi.org/10.1051/forest: 2007048.

[34] Gil Garrote, Herminia Domínguez, and Juan Carlos Parajó. Interpretation of deacetylation and hemicellulose hydrolysis during hydrothermal treatments on the basis of the severity factor. Process Biochemistry, 37(10):1067-1073, 2002. ISSN 1359-5113. doi: https://doi.org/10.1016/S0032-9592(01)00315-6. URL https: //www.sciencedirect.com/science/article/pii/S0032959201003156.

[35] G. Garrote, H. Domínguez, and J. C. Parajó. Hydrothermal processing of lignocellulosic materials. Holz als Roh- und Werkstoff, 57(3):191-202, 1999. doi: $10.1007 /$ s001070050039. URL https://doi .org/10.1007/s001070050039.

[36] Xiaowei Zhou, Wenjun Li, Ross Mabon, and Linda J. Broadbelt. A critical review on hemicellulose pyrolysis. Energy Technology, 5(1):52-79, 2017. doi: https://doi. org/10.1002/ente.201600327. URL https://onlinelibrary.wiley.com/doi/abs/ 10.1002/ente. 201600327.

[37] Juan Guo, Harald Rennhofer, Yafang Yin, and Helga C. Lichtenegger. The influence of thermo-hygro-mechanical treatment on the micro- and nanoscale architecture of 
wood cell walls using small- and wide-angle x-ray scattering. Cellulose, 23(4):23252340, 2016. doi: 10.1007/s10570-016-0982-2. URL https://doi.org/10.1007/ s10570-016-0982-2.

[38] Md. Tariqur Rabbani Bhuiyan, Nobuyuki Hirai, and Nobuo Sobue. Changes of crystallinity in wood cellulose by heat treatment under dried and moist conditions. Journal of Wood Science, 46(6):431-436, 2000. doi: 10.1007/BF00765800. URL https://doi .org/10.1007/BF00765800.

[39] Youichi Ito, Mitsuhiko Tanahashi, Mikiji Shigematsu, and Yoshihiko Shinoda. Compressive-molding of wood by high-pressure steam-treatment: Part 2. mechanism of permanent fixation. Holzforschung, 52(2):217-221, 1998. doi: doi:10.1515/ hfsg.1998.52.2.217. URL https://doi.org/10.1515/hfsg.1998.52.2.217.

[40] B. F. Tjeerdsma and H. Militz. Chemical changes in hydrothermal treated wood: Ftir analysis of combined hydrothermal and dry heat-treated wood. Holz als Rohund Werkstoff, 63(2):102-111, 2005. doi: 10.1007/s00107-004-0532-8. URL https: //doi.org/10.1007/s00107-004-0532-8.

[41] Seppo Andersson, Ritva Serimaa, Tiina Väänänen, Timo Paakkari, Saila Jämsä, and Pertti Viitaniemi. X-ray scattering studies of thermally modified scots pine (pinus sylvestris 1.). Holzforschung, 59(4):422-427, 2005. doi: doi:10.1515/HF.2005.069. URL https://doi.org/10.1515/HF.2005.069.

[42] Tetsuya Inagaki, Heinz W. Siesler, Katsuya Mitsui, and Satoru Tsuchikawa. Difference of the crystal structure of cellulose in wood after hydrothermal and aging degradation: A nir spectroscopy and xrd study. Biomacromolecules, 11(9):2300-2305, 09 2010. doi: 10.1021/bm100403y. URL https://doi.org/10.1021/bm100403y.

[43] N. L. Salmén and G. Back. The influence of water on the glass transition temperature of cellulose. Technical Association of the Pulp and Paper Industry, 60:137-140, 1977.

[44] Ludwik Szcześniak, Adam Rachocki, and Jadwiga Tritt-Goc. Glass transition temperature and thermal decomposition of cellulose powder. Cellulose, 15(3):445451, 2008. doi: 10.1007/s10570-007-9192-2. URL https://doi.org/10.1007/ s10570-007-9192-2. 
[45] M. Wentzel, M. Fleckenstein, T. Hofmann, and H. Militz. Relation of chemical and mechanical properties of eucalyptus nitens wood thermally modified in open and closed systems. Wood Material Science \& Engineering, 14(3):165-173, 2019. doi: 10.1080/17480272.2018.1450783. URL https://doi.org/10.1080/17480272. 2018.1450783 .

[46] Bruno Esteves and Helena Pereira. Wood modification by heat treatment: A review. BioResources, 4:370-404, 02 2009. doi: 10.15376/biores.4.1.370-404.

[47] F. W. Calonego, E. T. D. Severo, and A. W. Ballarin. Physical and mechanical properties of thermally modified wood from e. grandis. European Journal of Wood and Wood Products, 70(4):453-460, 2012. doi: 10.1007/s00107-011-0568-5. URL https://doi.org/10.1007/s00107-011-0568-5.

[48] Philipp Dietsch, Steffen Franke, Bettina Franke, Andreas Gamper, and Stefan Winter. Methods to determine wood moisture content and their applicability in monitoring concepts. Journal of Civil Structural Health Monitoring, 5(2):115-127, 2015. doi: 10.1007/s13349-014-0082-7. URL https://doi .org/10 .1007/s13349-014-0082-7.

[49] E. Obataya, T. Higashihara, and B. Tomita. Hygroscopicity of heat-treated wood iii. effect of steaming on the hygroscopicity of wood. Mokuzai Gakkaishi, 48:348-355, 012002.

[50] Michaela Eder, Olivier Arnould, John W. C. Dunlop, Joanna Hornatowska, and Lennart Salmén. Experimental micromechanical characterisation of wood cell walls. Wood Science and Technology, 47(1):163-182, 2013. doi: 10.1007/s00226-012-0515-6. URL https://doi .org/10.1007/s00226-012-0515-6.

[51] I. D. Cave. The longitudinal young's modulus of pinus radiata. Wood Science and Technology, 3(1):40-48, 1969. doi: 10.1007/BF00349983. URL https://doi.org/ 10.1007/BF00349983.

[52] J. M. Dinwoodie. Timber - review of the structure-mechanical property relationship. Journal of Microscopy, 104(1):3-32, 1975. doi: https://doi.org/10.1111/j. 1365-2818.1975.tb04002.x. URL https ://onlinelibrary. wiley . com/doi/abs/10. $1111 / j \cdot 1365-2818.1975 . t b 04002 . x$. 
[53] A. Reiterer, H. Lichtenegger, S. Tschegg, and P. Fratzl. Experimental evidence for a mechanical function of the cellulose microfibril angle in wood cell walls. Philosophical Magazine A, 79(9):2173-2184, 1999. doi: 10.1080/01418619908210415. URL https: //doi.org/10.1080/01418619908210415.

[54] Elsa M. L. Ehrnrooth and Petter Kolseth. The tensile testing of single wood pulp fibers in air and in water. Wood and Fiber Science, 16:549-566, 2007.

[55] DH Page, F El-Hosseiny, and K Winkler. Behaviour of single wood fibres under axial tensile strain. Nature, 229(5282):252-253, January 1971. ISSN 0028-0836. doi: 10.1038/229252a0. URL https://doi.org/10.1038/229252a0.

[56] I. Burgert, J. Keckes, K. Frühmann, P. Fratzl, and S. E. Tschegg. A comparison of two techniques for wood fibre isolation - evaluation by tensile tests on single fibres with different microfibril angle. Plant Biology, 4(1):9-12, 2002. doi: https: //doi.org/10.1055/s-2002-20430. URL https://onlinelibrary.wiley.com/doi/ $\mathrm{abs} / 10.1055 / \mathrm{s}-2002-20430$.

[57] P. C. Kersavage. Moisture content effect on tensile properties of individual douglas-fir latewood tracheids. Wood and Fiber Science, 5:105-117, 2007.

[58] Yan Yu, Zehui Jiang, Benhua Fei, Ge Wang, and Hankun Wang. An improved microtensile technique for mechanical characterization of short plant fibers: a case study on bamboo fibers. Journal of Materials Science, 46(3):739-746, 2011. doi: 10.1007/s10853-010-4806-8. URL https ://doi .org/10 .1007/s10853-010-4806-8.

[59] I. Burgert, M. Eder, K. Frühmann, J. Keckes, P. Fratzl, and S. Stanzl-tschegg. Properties of chemically and mechanically isolated fibres of spruce (picea abies [1.] karst.). part 3: Mechanical characterisation. volume 59, pages 354-357, 2005. doi: https://doi.org/10.1515/HF.2005.058.

[60] Robert Evans and Jugo Ilic. Rapid prediction of wood stiffness from microfibril angle and density. Forest Products Journal, 51:53-57, 032001.

[61] DH Page and F El-Hosseiny. mechanical properties of single wood pulp fibres. vi. fibril angle and the shape of the stress-strain curve. Pulp \& paper Canada, 1983. 
[62] Laurence Mott, S. Shaler, L. Groom, and B. Liang. The tensile testing of individual wood fibers using environmental scanning electron microscopy and video image analysis. Tappi Journal, 78:142-148, 1995.

[63] Michaela Eder, Nasko Terziev, Geoffrey Daniel, and Ingo Burgert. The effect of (induced) dislocations on the tensile properties of individual norway spruce fibres. Holzforschung, 62(1):77-81, 2008. doi: doi:10.1515/HF.2008.011. URL https:// doi.org/10.1515/HF.2008.011.

[64] Lisbeth G. Thygesen, Michaela Eder, and Ingo Burgert. Dislocations in single hemp fibres - investigations into the relationship of structural distortions and tensile properties at the cell wall level. Journal of Materials Science, 42(2):558-564, 2007. doi: 10.1007/s10853-006-1113-5. URL https : //doi .org/10 .1007/s10853-006-1113-5.

[65] Jozef Keckes, Ingo Burgert, Klaus Frühmann, Martin Müller, Klaas Kölln, Myles Hamilton, Manfred Burghammer, Stephan V. Roth, Stefanie Stanzl-Tschegg, and Peter Fratzl. Cell-wall recovery after irreversible deformation of wood. Nature Materials, 2(12):810-813, 2003. doi: 10.1038/nmat1019. URL https://doi.org/ $10.1038 /$ nmat 1019 .

[66] Cédric Montero, Bruno Clair, Tancréde Alméras, Arie van der Lee, and Joseph Gril. Relationship between wood elastic strain under bending and cellulose crystal strain. Composites Science and Technology, 72(2):175-181, 2012. ISSN 02663538. doi: https://doi.org/10.1016/j.compscitech.2011.10.014. URL https://www . sciencedirect.com/science/article/pii/S0266353811003800.

[67] Steffen Orso, Ulrike G. K. Wegst, and Eduard Arzt. The elastic modulus of spruce wood cell wall material measured by an in situ bending technique. Journal of Materials Science, 41(16):5122-5126, 2006. doi: 10.1007/s10853-006-0072-1. URL https://doi.org/10.1007/s10853-006-0072-1.

[68] Ramesh-Babu Adusumalli, Rejin Raghavan, Rudy Ghisleni, Tanja Zimmermann, and Johann Michler. Deformation and failure mechanism of secondary cell wall in spruce late wood. Applied Physics A, 100(2):447-452, 2010. doi: 10.1007/ s00339-010-5847-1. URL https://doi.org/10.1007/s00339-010-5847-1. 
[69] R. Wimmer, B. N. Lucas, W. C. Oliver, and T. Y. Tsui. Longitudinal hardness and young's modulus of spruce tracheid secondary walls using nanoindentation technique. Wood Science and Technology, 31(2):131-141, 1997. doi: 10.1007/BF00705928. URL https://doi.org/10.1007/BF00705928.

[70] W. Gindl, H. S. Gupta, T. Schöberl, H. C. Lichtenegger, and P. Fratzl. Mechanical properties of spruce wood cell walls by nanoindentation. Applied Physics A, 79 (8):2069-2073, 2004. doi: 10.1007/s00339-004-2864-y. URL https://doi.org/10. $1007 / \mathrm{s} 00339-004-2864-\mathrm{y}$.

[71] DH Page, F Elhosseiny, K Winkler, and APS Lancaster. Elastic modulus of single wood pulp fibers. Tappi, 60(4):114-117, 1977.

[72] Yan Wu, Siqun Wang, Dingguo Zhou, Cheng Xing, and Yang Zhang. Use of nanoindentation and silviscan to determine the mechanical properties of 10 hardwood species. Wood and fiber science: journal of the Society of Wood Science and Technology, 41:64-73, 012009.

[73] Johannes Konnerth, Notburga Gierlinger, Jozef Keckes, and Wolfgang Gindl. Actual versus apparent within cell wall variability of nanoindentation results from wood cell walls related to cellulose microfibril angle. Journal of Materials Science, 44 (16):4399-4406, 2009. doi: 10.1007/s10853-009-3665-7. URL https://doi .org/10. $1007 /$ s10853-009-3665-7.

[74] W Gindl, H S Gupta, and C Grünwald. Lignification of spruce tracheid secondary cell walls related to longitudinal hardness and modulus of elasticity using nanoindentation. Canadian Journal of Botany, 80(10):1029-1033, 2002. doi: 10.1139/ b02-091. URL https://doi.org/10.1139/b02-091.

[75] A. Bergander and L. Salmén. Cell wall properties and their effects on the mechanical properties of fibers. Journal of Materials Science, 37(1):151-156, 2002. doi: 10.1023/ A:1013115925679. URL https://doi.org/10.1023/A:1013115925679.

[76] Marc Borrega and Lorna J. Gibson. Mechanics of balsa (ochroma pyramidale) wood. Mechanics of Materials, 84:75-90, 2015. ISSN 0167-6636. doi: https://doi.org/ 10.1016/j.mechmat.2015.01.014. URL https://www.sciencedirect.com/science/ article/pii/S0167663615000216. 
[77] Lorna J. Gibson and Michael F. Ashby. Cellular Solids: Structure and Properties. Cambridge Solid State Science Series. Cambridge University Press, 2 edition, 1997. doi: $10.1017 / \mathrm{CBO} 9781139878326$.

[78] Kevin Menard. Dynamic Mechanical Analysis: A Practical Introduction, volume 02. CRC Press, 2 edition, 01 2008. doi: https://doi.org/10.1201/9781420053135.

[79] Xiaoning Tang and Xiong Yan. A review on the damping properties of fiber reinforced polymer composites. Journal of Industrial Textiles, 49(6):693-721, 2020. doi: 10. 1177/1528083718795914. URL https://doi .org/10.1177/1528083718795914.

[80] Christen Skaar. Wood-Water Relations. Springer Series in Wood Science. Springer, Berlin, Heidelberg, 1 edition, 1988. doi: https://doi.org/10.1007/978-3-642-73683-4.

[81] Yoichi Kojima and Hiroyuki Yamamoto. Effect of moisture content on the longitudinal tensile creep behavior of wood. Journal of Wood Science, 51(5):462-467, 2005. doi: 10.1007/s10086-004-0676-5. URL https ://doi .org/10 .1007/s10086-004-0676-5.

[82] Chika Takahashi, Yutaka Ishimaru, Ikuho Iida, and Yuzo Furuta. The creep of wood destabilized by change in moisture content. part 3: The influence of changing moisture history on creep behavior. Holzforschung, 60(3):299-303, 2006. doi: doi: 10.1515/HF.2006.048. URL https://doi .org/10.1515/HF . 2006. 048.

[83] Yujie Meng, Yuzhi Xia, Timothy M. Young, Zhiyong Cai, and Siqun Wang. Viscoelasticity of wood cell walls with different moisture content as measured by nanoindentation. RSC Adv., 5:47538-47547, 2015. doi: 10.1039/C5RA05822H. URL http://dx.doi.org/10.1039/C5RA05822H.

[84] J. Mukudai and S. Yata. Further modeling and simulation of viscoelastic behavior (bending deflection) of wood under moisture change. Wood Science and Technology, 21(1):49-63, 1987. doi: 10.1007/BF00349717. URL https://doi.org/10.1007/ BF00349717.

[85] Qingzheng Cheng and Siqun Wang. A method for testing the elastic modulus of single cellulose fibrils via atomic force microscopy. Composites Part A: Applied Science and Manufacturing, 39(12):1838-1843, 2008. ISSN 1359-835X. doi: https: 
//doi.org/10.1016/j.compositesa.2008.09.007. URL https://www.sciencedirect. com/science/article/pii/S1359835X08002352.

[86] Bruno Clair, Richard Arinero, Gérard Lévèque, Michel Ramonda, and Bernard Thibaut. Imaging the mechanical properties of wood cell layers by atomic force modulation microscopy. IAWA Journal, 24:223-230, 2003. URL https://hal. archives-ouvertes.fr/hal-00004541.

[87] Sandeep S. Nair, Siqun Wang, and Donna C. Hurley. Nanoscale characterization of natural fibers and their composites using contact-resonance force microscopy. Composites Part A: Applied Science and Manufacturing, 41(5):624-631, 2010. ISSN 1359-835X. doi: https://doi.org/10.1016/j.compositesa.2010.01.009. URL https: //www.sciencedirect.com/science/article/pii/S1359835X10000242.

[88] O. Arnould and R. Arinero. Towards a better understanding of wood cell wall characterisation with contact resonance atomic force microscopy. Composites Part A: Applied Science and Manufacturing, 74:69-76, 2015. ISSN 1359-835X. doi: https: //doi.org/10.1016/j.compositesa.2015.03.026. URL https://www.sciencedirect. com/science/article/pii/S1359835X15001220.

[89] Dan Ren, Hankun Wang, Zixuan Yu, Hao Wang, and Yan Yu. Mechanical imaging of bamboo fiber cell walls and their composites by means of peakforce quantitative nanomechanics (pqnm) technique. Holzforschung, 69(8):975-984, 2015. doi: doi: 10.1515/hf-2014-0237. URL https://doi.org/10.1515/hf-2014-0237.

[90] L. Muraille, V. Aguié-Béghin, B. Chabbert, and M. Molinari. Bioinspired lignocellulosic films to understand the mechanical properties of lignified plant cell walls at nanoscale. Scientific Reports, 7(1):44065, 2017. doi: 10.1038/srep44065. URL https://doi.org/10.1038/srep44065.

[91] Ingo Burgert. Exploring the micromechanical design of plant cell walls. American Journal of Botany, 93(10):1391-1401, 2006. doi: https://doi.org/10.3732/ajb.93.10. 1391. URL https://bsapubs.onlinelibrary.wiley.com/doi/abs/10.3732/ajb. 93.10 .1391$.

[92] Xinzhou Wang, Yanjun Li, Yuhe Deng, Wangwang Yu, Xuqin Xie, and Siqun Wang. Contributions of basic chemical components to the mechanical behavior of wood fiber 
cell walls as evaluated by nanoindentation. BioResources, 11(3):6026-6039, 2016. doi: 10.3732/ajb.93.10.1391.

[93] Freddy Muñoz, Paulina Valenzuela, and William Gacitúa. Eucalyptus nitens: nanomechanical properties of bark and wood fibers. Applied Physics A, 108(4):10071014, 2012. doi: 10.1007/s00339-012-7014-3. URL https://doi.org/10.1007/ s00339-012-7014-3.

[94] Isabel Carrillo-Varela, Paulina Valenzuela, William Gacitua, and Regis Mendonça. An evaluation of fiber biometry and nanomechanical properties of different eucalyptus species. Bioresources, 14:6433, 06 2019. doi: 10.15376/biores.14.3.6433-6446.

[95] W.T.Y. Tze, S. Wang, T.G. Rials, G.M. Pharr, and S.S. Kelley. Nanoindentation of wood cell walls: Continuous stiffness and hardness measurements. Composites Part A: Applied Science and Manufacturing, 38(3):945-953, 2007. ISSN 1359835X. doi: https://doi.org/10.1016/j.compositesa.2006.06.018. URL https://www . sciencedirect.com/science/article/pii/S1359835X06002077.

[96] J.E. Jakes, C.R. Frihart, J.F. Beecher, R.J. Moon, and D.S. Stone. Experimental method to account for structural compliance in nanoindentation measurements. Journal of Materials Research, 23(4):1113-1127, 2008. doi: 10.1557/jmr.2008.0131.

[97] Xinan Zhang, Qiuhong Zhao, Siqun Wang, Rosa Trejo, Edgar Lara-Curzio, and Guanben Du. Characterizing strength and fracture of wood cell wall through uniaxial micro-compression test. Composites Part A: Applied Science and Manufacturing, 41(5):632-638, 2010. ISSN 1359-835X. doi: https://doi.org/10. 1016/j.compositesa.2010.01.010. URL https://www. sciencedirect.com/science/ article/pii/S1359835X10000254.

[98] E.G. Herbert, W.C. Oliver, A. Lumsdaine, and G.M. Pharr. Measuring the constitutive behavior of viscoelastic solids in the time and frequency domain using flat punch nanoindentation. Journal of Materials Research, 24(3):626?637, 2009. doi: $10.1557 /$ jmr.2009.0089.

[99] Erik G. Herbert, P. Sudharshan Phani, and Kurt E. Johanns. Nanoindentation of viscoelastic solids: A critical assessment of experimental methods. Current Opinion in 
Solid State and Materials Science, 19(6):334-339, 2015. ISSN 1359-0286. doi: https: //doi.org/10.1016/j.cossms.2014.12.006. URL https://www.sciencedirect.com/ science/article/pii/S1359028614000965. Recent Advances in Nanoindentation.

[100] P.O. Guglielmi, E.G. Herbert, L. Tartivel, M. Behl, A. Lendlein, N. Huber, and E.T. Lilleodden. Mechanical characterization of oligo(ethylene glycol)-based hydrogels by dynamic nanoindentation experiments. Journal of the Mechanical Behavior of Biomedical Materials, 46:1-10, 2015. ISSN 1751-6161. doi: https://doi.org/10.1016/ j.jmbbm.2015.02.009. URL https://www.sciencedirect.com/science/article/ $\mathrm{pii/S175161611500051X.}$

[101] E G Herbert, W C Oliver, and G M Pharr. Nanoindentation and the dynamic characterization of viscoelastic solids. Journal of Physics D: Applied Physics, 41(7): 074021, mar 2008. doi: 10.1088/0022-3727/41/7/074021. URL https://doi.org/ 10.1088/0022-3727/41/7/074021.

[102] Sidney R Cohen and Estelle Kalfon-Cohen. Dynamic nanoindentation by instrumented nanoindentation and force microscopy: a comparative review. Beilstein journal of nanotechnology, 4:815-833, 11 2013. doi: 10.3762/bjnano.4.93. URL https://pubmed.ncbi.nlm.nih.gov/24367751.

[103] Tian Zhang, Shu-Lin Bai, Sandrine Bardet, Tancrède Alméras, Bernard Thibaut, and Jacques Beauchêne. Radial variations of vibrational properties of three tropical woods. Journal of Wood Science, 57(5):377-386, 2011. doi: 10.1007/ s10086-011-1189-7. URL https://doi.org/10.1007/s10086-011-1189-7.

[104] Yanjun Li, Liping Yin, Chengjian Huang, Yujie Meng, Feng Fu, Siqun Wang, and Qiang Wu. Quasi-static and dynamic nanoindentation to determine the influence of thermal treatment on the mechanical properties of bamboo cell walls. Holzforschung, 69(7):909-914, 2015. doi: doi:10.1515/hf-2014-0112. URL https://doi.org/10. 1515/hf-2014-0112.

[105] Meisam K. Habibi, Lik ho Tam, Denvid Lau, and Yang Lu. Viscoelastic damping behavior of structural bamboo material and its microstructural origins. Mechanics of Materials, 97:184-198, 2016. ISSN 0167-6636. doi: https://doi.org/10.1016/j. 
mechmat.2016.03.002. URL https://www.sciencedirect.com/science/article/ pii/S0167663616000521.

[106] Maximilian Wentzel. Process optimization of thermal modification of Chilean Eucalyptus nitens plantation wood. PhD thesis, Niedersächsische Staats- und Universitätsbibliothek Göttingen, Georg-August-Universität Göttingen, 2019. URL http://hdl .handle.net/11858/00-1735-0000-002E-E5A0-2.

[107] TAPPI. Solvent extractives of wood and pulp: T $204 \mathrm{~cm}-97$. Chemical Properties Committee of the Process and Product Quality Division, 1997. URL https:// research.cnr.ncsu. edu/wpsanalytical/documents/T204.PDF.

[108] L. E. Wise, M. Murphy, and A. A. D. Adieco. A chlorite holocellulose, its fractionation and bearing on summative wood analysis and studies on the hemicelluloses. 1946.

[109] TAPPI. Alpha-, beta- and gamma-cellulose in pulp: T 203 cm-99. Chemical Properties Committee of the Process and Product Quality Division, 1999. URL https://research.cnr.ncsu. edu/wpsanalytical/documents/T203.PDF.

[110] Amie Sluiter, Bonnie Hames, R. Ruiz, C. Scarlata, J. Sluiter, David Templeton, and D. Crocker. Determination of structural carbohydrates and lignin in biomass, in: Laboratory analytical procedure (lap). National Renewable Energy Laboratory, 01 2008.

[111] Yujie Meng. Methods for characterizing mechanical properties of wood cell walls via nanoindentation, 2010. URL https://trace.tennessee.edu/utk_gradthes/731.

[112] Wim Willems. A novel economic large-scale production technology for high-quality thermally modified wood. European Conference on Wood Modification, pages 31-35, 04 2009. URL http://costfp1303.iam.upr.si/en/resources/files/ publications/ecwm4-oral-1/3willems.pdf.

[113] Maximilian Wentzel, Christian Brischke, and Holger Militz. Dynamic and static mechanical properties of Eucalyptus nitens thermally modified in an open and closed 
reactor system. Maderas. Ciencia y tecnologÃa, 21:141 - 152, 03 2019. ISSN 0718221X. URL https://scielo.conicyt.cl/scielo.php?script=sci_arttext\&pid= $\mathrm{S} 0718-221 \mathrm{X} 2019000200141 \& \mathrm{nrm}=$ iso.

[114] Michael Altgen and Holger Militz. Influence of process conditions on hygroscopicity and mechanical properties of european beech thermally modified in a high-pressure reactor system. Holzforschung, 70(10):971-979, 2016. doi: doi:10.1515/hf-2015-0235. URL https://doi .org/10.1515/hf-2015-0235.

[115] Duygu Kocaefe, Sandor Poncsak, Geneviève Doré, and Ramdane Younsi. Effect of heat treatment on the wettability of white ash and soft maple by water. Holz als Roh- und Werkstoff, 66(5):355-361, 2008. doi: 10.1007/s00107-008-0233-9. URL https://doi .org/10.1007/s00107-008-0233-9.

[116] Duygu Kocaefe, Sandor Poncsak, and Yaman Boluk. Effect of thermal treatment on the chemical composition and mechanical properties of birch and aspen. BioResources, 3:517-537, 052008.

[117] Michael Altgen, Wim Willems, and Holger Militz. Wood degradation affected by process conditions during thermal modification of european beech in a highpressure reactor system. European Journal of Wood and Wood Products, 74(5): 653-662, 2016. doi: 10.1007/s00107-016-1045-y. URL https://doi.org/10.1007/ s00107-016-1045-y.

[118] Dong Xing, Jian Li, and Siqun Wang. Comparison of the chemical and micromechanical properties of larix spp. after eco-friendly heat treatments measured by in situ nanoindentation. Scientific Reports, 10(1):4358, 2020. doi: 10.1038/ s41598-020-61314-6. URL https://doi .org/10 .1038/s41598-020-61314-6.

[119] W.C. Oliver and G.M. Pharr. An improved technique for determining hardness and elastic modulus using load and displacement sensing indentation experiments. Journal of Materials Research, 7(6):1564?1583, 1992. doi: 10.1557/JMR.1992.1564.

[120] S. A. Syed Asif, K. J. Wahl, and R. J. Colton. Nanoindentation and contact stiffness measurement using force modulation with a capacitive load-displacement transducer. Review of Scientific Instruments, 70(5):2408-2413, 1999. doi: 10.1063/1.1149769. URL https://doi .org/10.1063/1.1149769. 
[121] G. M. Odegard, T. S. Gates, and H. M. Herring. Characterization of viscoelastic properties of polymeric materials through nanoindentation. Experimental Mechanics, 45(2):130-136, 2005. doi: 10.1007/BF02428185. URL https://doi.org/10.1007/ BF02428185.

[122] J. Hay, P. Agee, and E. Herbert. Continuous stiffness measurement during instrumented indentation testing. Experimental Techniques, 34(3):86-94, 2010. doi: 10.1111/j.1747-1567.2010.00618.x. URL https://doi .org/10.1111/j.1747-1567. $2010.00618 . x$.

[123] Lei Wang and Xianping Liu. Characterization of viscoelastic materials by quasistatic and dynamic indentation. Measurement Science and Technology, 25(6):064017, apr 2014. doi: 10.1088/0957-0233/25/6/064017. URL https://doi.org/10.1088/ $0957-0233 / 25 / 6 / 064017$.

[124] Julia Deuschle, Susan Enders, and Eduard Arzt. Surface detection in nanoindentation of soft polymers. Journal of Materials Research, 22(11):3107-3119, 2007. doi: 10. 1557/JMR.2007.0394.

[125] George M. Pharr, Erik G. Herbert, and Yanfei Gao. The indentation size effect: A critical examination of experimental observations and mechanistic interpretations. Annual Review of Materials Research, 40(1):271-292, 2010. doi: 10.1146/annurev-matsci-070909-104456. URL https://doi.org/10.1146/ annurev-matsci-070909-104456.

[126] K. L. Johnson. Contact Mechanics. Cambridge University Press, 1985. doi: 10.1017/ CBO9781139171731.

[127] M. Mata, O. Casals, and J. Alcalá. The plastic zone size in indentation experiments: The analogy with the expansion of a spherical cavity. International Journal of Solids and Structures, 43(20):5994-6013, 2006. ISSN 0020-7683. doi: https://doi.org/10.1016/j.ijsolstr.2005.07.002. URL https://www.sciencedirect. $\mathrm{com} / \mathrm{science/article/pii/S0020768305004476.}$

[128] P. Sudharshan Phani and W.C. Oliver. A critical assessment of the effect of indentation spacing on the measurement of hardness and modulus using instrumented 
indentation testing. Materials \& Design, 164:107563, 2019. ISSN 0264-1275. doi: https://doi.org/10.1016/j.matdes.2018.107563. URL https ://www . sciencedirect. com/science/article/pii/S0264127518309274.

[129] Johannes Konnerth and Wolfgang Gindl. Mechanical characterisation of woodadhesive interphase cell walls by nanoindentation. Holzforschung, 60(4):429-433, 2006. doi: doi:10.1515/HF.2006.067. URL https://doi.org/10.1515/HF. 2006. 067.

[130] B. Lachenbruch, G. R. Johnson, G. M. Downes, and R. Evans. Relationships of density, microfibril angle, and sound velocity with stiffness and strength in mature wood of douglas-fir. Canadian Journal of Forest Research, 40(1):55-64, 2010. doi: 10.1139/X09-174. URL https://doi.org/10.1139/X09-174.

[131] Leopold Wagner, Thomas K. Bader, David Auty, and Karin de Borst. Key parameters controlling stiffness variability within trees: a multiscale experimentalnumerical approach. Trees, 27(1):321-336, 2013. doi: 10.1007/s00468-012-0801-9. URL https://doi .org/10.1007/s00468-012-0801-9.

[132] Xinzhou Wang, Xuanzong Chen, Xuqin Xie, Yan Wu, Linguo Zhao, Yanjun Li, and Siqun Wang. Effects of thermal modification on the physical, chemical and micromechanical properties of masson pine wood (pinus massoniana lamb.). Holzforschung, 72(12):1063-1070, 2018. doi: doi:10.1515/hf-2017-0205. URL https://doi.org/ 10.1515/hf-2017-0205.

[133] Dong Xing, Jian Li, Xinzhou Wang, and Siqun Wang. In situ measurement of heattreated wood cell wall at elevated temperature by nanoindentation. Industrial Crops and Products, 87:142-149, 2016. ISSN 0926-6690. doi: https://doi.org/10.1016/ j.indcrop.2016.04.017. URL https://www. sciencedirect.com/science/article/ pii/S0926669016302278.

[134] Yanjun Li, Chengjian Huang, Li Wang, Siqun Wang, and Xinzhou Wang. The effects of thermal treatment on the nanomechanical behavior of bamboo (phyllostachys pubescens mazel ex h. de lehaie) cell walls observed by nanoindentation, xrd, and wet chemistry. Holzforschung, 71(2):129-135, 2017. doi: doi:10.1515/hf-2016-0124. URL https://doi .org/10.1515/hf-2016-0124. 
[135] D. Hull and T. W. Clyne. An Introduction to Composite Materials. Cambridge Solid State Science Series. Cambridge University Press, 2 edition, 1996. doi: 10. $1017 /$ CBO9781139170130.

[136] W. J. Cousins. Elastic modulus of lignin as related to moisture content. Wood Science and Technology, 10(1):9-17, 1976. doi: 10.1007/BF00376380. URL https: //doi.org/10.1007/BF00376380.

[137] R. J. Astley, K. A. Stol, and J. J. Harrington. Modelling the elastic properties of softwood. Holz als Roh- und Werkstoff, 56(1):43-50, 1998. doi: 10.1007/s001070050262. URL https://doi.org/10.1007/s001070050262.

[138] Jörg Fromm, Beate Rockel, Silke Lautner, Elisabeth Windeisen, and Gerhard Wanner. Lignin distribution in wood cell walls determined by tem and backscattered sem techniques. Journal of Structural Biology, 143(1):77-84, 2003. ISSN 10478477. doi: https://doi.org/10.1016/S1047-8477(03)00119-9. URL https://www . sciencedirect.com/science/article/pii/S1047847703001199.

[139] I. D. Cave. The anisotropic elasticity of the plant cell wall. Wood Science and Technology, 2(4):268-278, 1968. doi: 10.1007/BF00350273. URL https://doi. org/10.1007/BF00350273.

[140] Paulo Ricardo Gherardi Hein and José Tarcísio Lima. Relationships between microfibril angle, modulus of elasticity and compressive strength in Eucalyptus wood. Maderas. Ciencia y tecnología, 14:267 - 274, 11 2012. ISSN 0718221X. URL https://scielo. conicyt.cl/scielo.php?script=sci_arttext\&pid= $\mathrm{S} 0718-221 \mathrm{X} 2012000300002 \mathrm{\& nrm}=$ iso.

[141] Lorna J. Gibson. The hierarchical structure and mechanics of plant materials. Journal of The Royal Society Interface, 9(76):2749-2766, 2012. doi: 10.1098/rsif. 2012.0341. URL https://royalsocietypublishing.org/doi/abs/10.1098/rsif . 2012.0341.

[142] Ulrich Müller, Sretenovic A., WA Gindl, and Alfred Teischinger. Longitudinal shear properties of europe larch wood related to cell-wall structure. Wood and fiber science: journal of the Society of Wood Science and Technology, 36:143-151, 042004. 
[143] R.D. Adams and D.G.C. Bacon. Effect of fibre orientation and laminate geometry on the dynamic properties of cfrp. Journal of Composite Materials, 7(4):402428, 1973. doi: 10.1177/002199837300700401. URL https://doi.org/10.1177/ 002199837300700401.

[144] Noritsugu Terashima, Tatsuya Awano, Keiji Takabe, and Masato Yoshida. Formation of macromolecular lignin in ginkgo xylem cell walls as observed by field emission scanning electron microscopy. Comptes Rendus Biologies, 327(9):903910, 2004. ISSN 1631-0691. doi: https://doi.org/10.1016/j.crvi.2004.08.001. URL https://www. sciencedirect.com/science/article/pii/S1631069104001738.

[145] David Labonte, Anne-Kristin Lenz, and Michelle L. Oyen. On the relationship between indentation hardness and modulus, and the damage resistance of biological materials. Acta Biomaterialia, 57:373-383, 2017. ISSN 1742-7061. doi: https://doi.org/10.1016/j.actbio.2017.05.034. URL https://www.sciencedirect. com/science/article/pii/S174270611730315X.

[146] Edward Roszyk, Przemyslaw Mania, and Waldemar Moliński. The influence of microfibril angle on creep scotch pine wood under tensile stress along the grains. Wood research, 57:347-358, 052011.

[147] Lloyd Donaldson. Microfibril angle: Measurement, variation and relationships - a review. IAWA Journal, 29(4):345 - 386, 01 Jan. 2008. doi: https://doi.org/10. 1163/22941932-90000192. URL https://brill.com/view/journals/iawa/29/4/ article-p345_1.xml.

[148] Daniel Casellas, Jaume Caro, Sílvia Molas, José M. Prado, and Isaac Valls. Fracture toughness of carbides in tool steels evaluated by nanoindentation. Acta Materialia, 55(13):4277-4286, 2007. ISSN 1359-6454. doi: https://doi.org/10.1016/ j.actamat.2007.03.028. URL https://www. sciencedirect.com/science/article/ pii/S1359645407002340.

[149] A. Bolshakov and G. M. Pharr. Influences of pileup on the measurement of mechanical properties by load and depth sensing indentation techniques. Journal of Materials

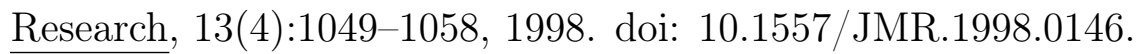


[150] M. Sakai. The meyer hardness: A measure for plasticity? Journal of Materials Research, 14(9):3630?3639, 1999. doi: 10.1557/JMR.1999.0490.

[151] Yang-Tse Cheng and Che-Min Cheng. Relationships between hardness, elastic modulus, and the work of indentation. Applied Physics Letters, 73(5):614-616, 1998. doi: 10.1063/1.121873. URL https://doi.org/10.1063/1.121873.

[152] Yoshiharu Nishiyama, Paul Langan, Hugh O'Neill, Sai Venkatesh Pingali, and Shane Harton. Structural coarsening of aspen wood by hydrothermal pretreatment monitored by small- and wide-angle scattering of x-rays and neutrons on oriented specimens. Cellulose, 21(2):1015-1024, 2014. doi: 10.1007/s10570-013-0069-2. URL https://doi.org/10.1007/s10570-013-0069-2.

[153] Roger H. Newman. Crystalline forms of cellulose in softwoods and hardwoods. Journal of Wood Chemistry and Technology, 14(3):451-466, 1994. doi: 10.1080/ 02773819408003107 . URL https://doi .org/10.1080/02773819408003107.

[154] Hiroyuki Yamamoto and Fumitaka Horii. Cpmas carbon-13 nmr analysis of the crystal transformation induced for valonia cellulose by annealing at high temperatures. Macromolecules, 26(6):1313-1317, 03 1993. doi: 10.1021/ma00058a020. URL https://doi.org/10.1021/ma00058a020.

[155] Jean Francois Dumail and Lennart Salmén. Compression behaviour of saturated wood perpendicular to grain under large deformations. comparison between watersaturated and ethylene glycol-saturated wood. Holzforschung, 51(4):296-302, 1997. doi: doi:10.1515/hfsg.1997.51.4.296. URL https://doi.org/10.1515/hfsg. 1997. 51.4 .296$.

[156] R Chandra, S.P Singh, and K Gupta. Damping studies in fiber-reinforced composites - a review. Composite Structures, 46(1):41-51, 1999. ISSN 0263-8223. doi: https:// doi.org/10.1016/S0263-8223(99)00041-0. URL https://www.sciencedirect.com/ science/article/pii/S0263822399000410.

[157] M. Kaliske and H. Rothert. Damping characterization of unidirectional fibre reinforced polymer composites. Composites Engineering, 5(5):551-567, 1995. ISSN 0961-9526. doi: https://doi.org/10.1016/0961-9526(95)00028-L. URL https : //www . sciencedirect.com/science/article/pii/096195269500028L. 
[158] M.S. Sweet and J.E. Winandy. Influence of degree of polymerization of cellulose and hemicellulose on strength loss in fire-retardant-treated southern pine. Holzforschung, 53(3):311-317, 1999. doi: doi:10.1515/HF.1999.051. URL https://doi.org/10. $1515 / \mathrm{HF} .1999 .051$.

[159] S. Levan, R. Ross, and J. Winandy. Effects of fire retardant chemicals on the bending properties of wood at elevated temperatures, volume 498. 1990. URL https://www . fpl.fs.fed.us/documnts/fplrp/fplrp498.pdf.

[160] Gerard T. Pott. Natural Fibers with Low Moisture Sensitivity, pages 105122. Springer US, Boston, MA, 2004. ISBN 978-1-4419-9050-1. doi: 10.1007/ 978-1-4419-9050-1_8. URL https://doi.org/10.1007/978-1-4419-9050-1_8.

[161] A. Pizzi, A. Stephanou, M.J. Boonstra, and A.J. Pendlebury. A new concept on the chemical modification of wood by organic anhydrides. Holzforschung, 48(s1):91-94, 1994. doi: doi:10.1515/hfsg.1994.48.s1.91. URL https://doi.org/10.1515/hfsg. 1994.48.s1.91.

[162] Miro Suchy, Eero Kontturi, and Tapani Vuorinen. Impact of drying on wood ultrastructure: Similarities in cell wall alteration between native wood and isolated wood-based fibers. Biomacromolecules, 11(8):2161-2168, 08 2010. doi: 10.1021/bm100547n. URL https://doi.org/10.1021/bm100547n.

[163] J. Heijboer. Dynamic mechanical properties and impact strength. Journal of Polymer Science Part C: Polymer Symposia, 16(7):3755-3763, 1967. doi: https://doi.org/ 10.1002/polc.5070160716. URL https ://onlinelibrary. wiley.com/doi/abs/10. 1002/polc. 5070160716 .

[164] Yoshitaka Kubojima, Takeshi Okano, and Masamitsu Ohta. Bending strength and toughness of heat-treated wood. Journal of Wood Science, 46(1):8-15, 2000. doi: 10.1007/BF00779547. URL https://doi.org/10.1007/BF00779547.

[165] Andreas Otto Rapp, Christian Brischke, and Christian Robert Welzbacher. Interrelationship between the severity of heat treatments and sieve fractions after impact ball milling: a mechanical test for quality control of thermally modified wood. Holzforschung, 60(1):64-70, 2006. doi: doi:10.1515/HF.2006.012. URL https://doi.org/10.1515/HF.2006.012. 
[166] Ping Xu, Lloyd A. Donaldson, Zachary R. Gergely, and L. Andrew Staehelin. Dualaxis electron tomography: a new approach for investigating the spatial organization of wood cellulose microfibrils. Wood Science and Technology, 41(2):101, 2006. doi: 10.1007/s00226-006-0088-3. URL https : //doi .org/10 . 1007/s00226-006-0088-3.

[167] James D. Kubicki, Hui Yang, Daisuke Sawada, Hugh O’Neill, Daniel Oehme, and Daniel Cosgrove. The shape of native plant cellulose microfibrils. Scientific Reports, 8(1):13983, 2018. doi: 10.1038/s41598-018-32211-w. URL https://doi.org/10. 1038/s41598-018-32211-w.

[168] L.A. Donaldson and A.P. Singh. Bridge-like structures between cellulose microfibrils in radiata pine (pinus radiata d. don) kraft pulp and holocellulose. Holzforschung, 52(5):449-454, 1998. doi: doi:10.1515/hfsg.1998.52.5.449. URL https://doi .org/ 10.1515/hfsg.1998.52.5.449.

[169] H. F. Jakob, D. Fengel, S. E. Tschegg, and P. Fratzl. The elementary cellulose fibril in picea abies: Comparison of transmission electron microscopy, small-angle $\mathrm{x}$-ray scattering, and wide-angle x-ray scattering results. Macromolecules, 28(26): 8782-8787, 12 1995. doi: 10.1021/ma00130a010. URL https://doi.org/10.1021/ ma00130a010.

[170] H.F. Jakob, P. Fratzl, and S.E. Tschegg. Size and arrangement of elementary cellulose fibrils in wood cells: A small-angle x-ray scattering study of picea abies. Journal of Structural Biology, 113(1):13-22, 1994. ISSN 1047-8477. doi: https://doi.org/10. 1006/jsbi.1994.1028. URL https://www.sciencedirect.com/science/article/ $\mathrm{pii} / \mathrm{S} 1047847784710288$.

[171] Jonas Hafrén, Takeshi Fujino, and Takao Itoh. Changes in Cell Wall Architecture of Differentiating Tracheids of Pinus thunbergii during Lignification. Plant and Cell Physiology, 40(5):532-541, 01 1999. ISSN 0032-0781. doi: 10.1093/oxfordjournals. pcp.a029574. URL https://doi .org/10.1093/oxfordjournals.pcp.a029574.

[172] Tatsuya Awano, Keiji Takabe, Minoru Fujita, and Geoffrey Daniel. Deposition of glucuronoxylans on the secondary cell wall of japanese beech as observed by immuno-scanning electron microscopy. Protoplasma, 212(1):72-79, 2000. doi: 10.1007/BF01279348. URL https://doi.org/10.1007/BF01279348. 
[173] Geoffrey Daniel and Isabelle Duchesne. Revealing the surface ultrastructure of spruce pulp fibres using field emission-sem. 7th International Conference on Biotechnology in the Pulp and Paper Industry, page B81, 06 1998. doi: 10.13140/2.1.4697.6645.

[174] S. Bardage, L. Donaldson, C. Tokoh, and G. Daniel. Ultrastructure of the cell wall of unbeaten norway spruce pulp fibre surfaces. Nordic Pulp \& Paper Research Journal, 19(4):448-452, 2004. doi: doi:10.3183/npprj-2004-19-04-p448-452. URL https://doi .org/10.3183/npprj-2004-19-04-p448-452.

[175] Geoffrey Daniel, Isabelle Duchesne, Chisuzu Tokoh, and Stig Bardage. The surface and intracellular nanostructure of wood fibres: electron microscope methods and applications, pages 87-104. 01 2004. ISBN 91-576-6803-5.

[176] Geoffrey Daniel, Jindrich Volc, and Marja-Leena Niku-Paavola. Cryo-fe-sem \& tem immuno-techniques reveal new details for understanding white-rot decay of lignocellulose. Comptes Rendus Biologies, 327(9):861-871, 2004. ISSN 1631-0691. doi: https://doi.org/10.1016/j.crvi.2004.08.003. URL https://www.sciencedirect. com/science/article/pii/S1631069104001763.

[177] Lloyd Donaldson. Cellulose microfibril aggregates and their size variation with cell wall type. Wood Science and Technology, 41(5):443, 2007. doi: 10.1007/ s00226-006-0121-6. URL https://doi.org/10.1007/s00226-006-0121-6.

[178] D Fengel. Ideas on the ultrastructural organization of the cell wall components. In Journal of Polymer Science Part C: Polymer Symposia.

[179] Eva-Lena Hult, Per Tomas Larsson, and Tommy Iversen. A cp/mas 13c-nmr study of supermolecular changes in the cellulose and hemicellulose structure during kraft pulping. Nordic Pulp \& Paper Research Journal, 16(1):33-39, 2001. doi: doi:10.3183/npprj-2001-16-01-p033-039. URL https://doi.org/10.3183/ npprj-2001-16-01-p033-039.

[180] E.-L. Hult, P.T. Larsson, and T. Iversen. Cellulose fibril aggregation - an inherent property of kraft pulps. Polymer, 42(8):3309-3314, 2001. ISSN 00323861. doi: https://doi.org/10.1016/S0032-3861(00)00774-6. URL https://www . sciencedirect.com/science/article/pii/S0032386100007746. 
[181] Isabelle Duchesne, Eva Hult, Ulrika Molin, Geoffrey Daniel, Tommy Iversen, and Helena Lennholm. The influence of hemicellulose on fibril aggregation of kraft pulp fibres as revealed by fe-sem and cp/mas 13c-nmr. Cellulose, 8(2):103-111, 2001. doi: 10.1023/A:1016645809958. URL https://doi .org/10.1023/A:1016645809958.

[182] Lloyd Donaldson, Jamie Hague, and Rebecca Snell. Lignin distribution in coppice poplar, linseed and wheat straw. Holzforschung, 55(4):379-385, 2001. doi: doi: 10.1515/HF.2001.063. URL https://doi.org/10.1515/HF .2001.063.

[183] B. J. Fergus, A. R. Procter, J. A. N. Scott, and D. A. I. Goring. The distribution of lignin in sprucewood as determined by ultraviolet microscopy. Wood Science and Technology, 3(2):117-138, 1969. doi: 10.1007/BF00639636. URL https://doi .org/ $10.1007 / \mathrm{BF} 00639636$.

[184] J. A. N. Scott, A. R. Procter, B. J. Fergus, and D. A. I. Goring. The application of ultraviolet microscopy to the distribution of lignin in wood description and validity of the technique. Wood Science and Technology, 3(1):73-92, 1969. doi: 10.1007/ BF00349985. URL https://doi.org/10.1007/BF00349985.

[185] S. Saka, P. Whiting, K. Fukazawa, and D. A. I. Goring. Comparative studies on lignin distribution by uv microscopy and bromination combined with edxa. Wood Science and Technology, 16(4):269-277, 1982. doi: 10.1007/BF00353151. URL https:// doi .org/10.1007/BF00353151.

[186] U. Westermark, O. Lidbrandt, and I. Eriksson. Lignin distribution in spruce (picea abies) determined by mercurization with sem-edxa technique. Wood Science and Technology, 22(3):243-250, 1988. doi: 10.1007/BF00386019. URL https://doi. org/10.1007/BF00386019.

[187] D. E. Bland, R. C. Foster, and A. F. Logan. The mechanism of permanganate and osmium tetroxide fixation and the distribution of lignin in the cell wall of pinus radiata. Holzforschung, 25(5):137-143, 1971. doi: doi:10.1515/hfsg.1971.25.5.137. URL https://doi .org/10.1515/hfsg.1971.25.5.137.

[188] J. Fahlén and L. Salmén. On the lamellar structure of the tracheid cell wall. Plant Biology, 4(3):339-345, 2002. doi: https://doi.org/10.1055/s-2002-32341. URL https://onlinelibrary.wiley.com/doi/abs/10.1055/s-2002-32341. 
[189] Jesper Fahlén and Lennart Salmén. Pore and matrix distribution in the fiber wall revealed by atomic force microscopy and image analysis. Biomacromolecules, 6(1): 433-438, 01 2005. doi: 10.1021/bm040068x. URL https://doi.org/10.1021/ bm040068x.

[190] Kirstin Casdorff, Tobias Keplinger, and Ingo Burgert. Nano-mechanical characterization of the wood cell wall by afm studies: comparison between ac- and qi ${ }^{\top M}$ mode. Plant Methods, 13(1):60, 2017. doi: 10.1186/s13007-017-0211-5. URL https://doi.org/10.1186/s13007-017-0211-5.

[191] Zahra Naghizadeh Mahani and Mehdi Tajvidi. Viscoelastic mapping of sprucepolyurethane bond line area using am-fm atomic force microscopy. International Journal of Adhesion and Adhesives, 79:59-66, 2017. ISSN 0143-7496. doi: https: //doi.org/10.1016/j.ijadhadh.2017.09.005. URL https : //www. sciencedirect.com/ science/article/pii/S0143749617301641.

[192] Olivier Arnould, David Siniscalco, Alain Bourmaud, Antoine Le Duigou, and Christophe Baley. Better insight into the nano-mechanical properties of flax fibre cell walls. Industrial Crops and Products, 97:224-228, 2017. ISSN 0926-6690. doi: https://doi.org/10.1016/j.indcrop.2016.12.020. URL https ://www.sciencedirect. com/science/article/pii/S092666901630855X.

[193] Marta Kocun, Aleksander Labuda, Waiman Meinhold, Irène Revenko, and Roger Proksch. Fast, high resolution, and wide modulus range nanomechanical mapping with bimodal tapping mode. ACS Nano, 11(10):10097-10105, 10 2017. doi: 10.1021/ acsnano.7b04530. URL https://doi.org/10.1021/acsnano.7b04530.

[194] Tomás R. Rodríguez and Ricardo García. Compositional mapping of surfaces in atomic force microscopy by excitation of the second normal mode of the microcantilever. Applied Physics Letters, 84(3):449-451, 2004. doi: 10.1063/1.1642273. URL https://doi.org/10.1063/1.1642273.

[195] John E. Sader, Julian A. Sanelli, Brian D. Adamson, Jason P. Monty, Xingzhan Wei, Simon A. Crawford, James R. Friend, Ivan Marusic, Paul Mulvaney, and Evan J. Bieske. Spring constant calibration of atomic force microscope cantilevers 
of arbitrary shape. Review of Scientific Instruments, 83(10):103705, 2012. doi: 10.1063/1.4757398. URL https://doi .org/10.1063/1.4757398.

[196] Aleksander Labuda, Marta Kocun, Martin Lysy, Tim Walsh, Jieh Meinhold, Tania Proksch, Waiman Meinhold, Caleb Anderson, and Roger Proksch. Calibration of higher eigenmodes of cantilevers. Review of Scientific Instruments, 87(7):073705, 2016. doi: 10.1063/1.4955122. URL https://aip.scitation.org/doi/abs/10. $1063 / 1.4955122$.

[197] Aleksander Labuda. Daniell method for power spectral density estimation in atomic force microscopy. Review of Scientific Instruments, 87(3):033704, 2016. doi: 10.1063/ 1.4943292. URL https://doi.org/10.1063/1.4943292.

[198] Jeffrey L. Hutter and John Bechhoefer. Calibration of atomic-force microscope tips.

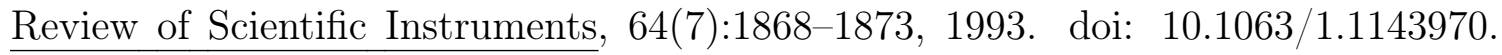
URL https://doi.org/10.1063/1.1143970.

[199] M. J. Higgins, R. Proksch, J. E. Sader, M. Polcik, S. Mc Endoo, J. P. Cleveland, and S. P. Jarvis. Noninvasive determination of optical lever sensitivity in atomic force microscopy. Review of Scientific Instruments, 77(1):013701, 2006. doi: 10.1063/1. 2162455. URL https://doi.org/10.1063/1.2162455.

[200] H J Butt and M Jaschke. Calculation of thermal noise in atomic force microscopy. Nanotechnology, 6(1):1-7, jan 1995. doi: 10.1088/0957-4484/6/1/001. URL https: //doi.org/10.1088/0957-4484/6/1/001.

[201] Ricardo Garcia and Roger Proksch. Nanomechanical mapping of soft matter by bimodal force microscopy. European Polymer Journal, 49(8):1897-1906, 2013. ISSN 0014-3057. doi: https://doi.org/10.1016/j.eurpolymj.2013.03.037. URL https:// www. sciencedirect.com/science/article/pii/S0014305713001699.

[202] Ricardo García. Amplitude Modulation Atomic Force Microscopy. John Wiley \& Sons, Ltd. ISBN 9783527632183.

[203] Roger Proksch, Marta Kocun, Donna Hurley, Mario Viani, Aleks Labuda, Waiman Meinhold, and Jason Bemis. Practical loss tangent imaging with amplitude- 
modulated atomic force microscopy. Journal of Applied Physics, 119(13):134901, 2016. doi: 10.1063/1.4944879. URL https://doi.org/10.1063/1.4944879.

[204] Aleksander Labuda, Marta Kocun, Waiman Meinhold, Deron Walters, and Roger Proksch. Generalized hertz model for bimodal nanomechanical mapping. Beilstein journal of nanotechnology, 7:970-982, 07 2016. doi: 10.3762/bjnano.7.89. URL https://pubmed.ncbi.nlm.nih.gov/27547614.

[205] F. J. Giessibl. A direct method to calculate tip-sample forces from frequency shifts in frequency-modulation atomic force microscopy. Applied Physics Letters, 78(1):123125, 2001. doi: 10.1063/1.1335546. URL https://doi.org/10.1063/1.1335546.

[206] E. Sahagún, P. García-Mochales, G. M. Sacha, and J. J. Sáenz. Energy dissipation due to capillary interactions: Hydrophobicity maps in force microscopy. Phys. Rev. Lett., 98:176106, Apr 2007. doi: 10.1103/PhysRevLett.98.176106. URL https: //link.aps.org/doi/10.1103/PhysRevLett.98.17616.

[207] L. Zitzler, S. Herminghaus, and F. Mugele. Capillary forces in tapping mode atomic force microscopy. Phys. Rev. B, 66:155436, Oct 2002. doi: 10.1103/PhysRevB.66. 155436. URL https://link.aps.org/doi/10.1103/PhysRevB.66.155436.

[208] Martin Felhofer, Peter Bock, Adya Singh, Batirtze Prats-Mateu, Ronald Zirbs, and Notburga Gierlinger. Wood deformation leads to rearrangement of molecules at the nanoscale. Nano Letters, 20(4):2647-2653, 04 2020. doi: 10.1021/acs.nanolett. 0c00205. URL https://doi.org/10.1021/acs.nanolett.0c00205.

[209] Claudia Gusenbauer, Devon S. Jakob, Xiaoji G. Xu, Dmitri V. Vezenov, Étienne Cabane, and Johannes Konnerth. Nanoscale chemical features of the natural fibrous material wood. Biomacromolecules, 21(10):4244-4252, 10 2020. doi: 10.1021/acs. biomac.0c01028. URL https://doi.org/10.1021/acs.biomac.0c01028.

[210] F. Golek, P. Mazur, Z. Ryszka, and S. Zuber. Afm image artifacts. Applied Surface Science, 304:11-19, 2014. ISSN 0169-4332. doi: https://doi.org/10.1016/ j.apsusc.2014.01.149. URL https://www.sciencedirect.com/science/article/ pii/S0169433214002013. Selected Papers from the 6th International Workshop on Surface Physics "Functional Materials". 
[211] A N Heyn. The microcrystalline structure of cellulose in cell walls of cotton, ramie, and jute fibers as revealed by negative staining of sections. The Journal of cell biology, 29(2):181-197, May 1966. ISSN 0021-9525 (Print); 1540-8140 (Electronic); 0021-9525 (Linking). doi: 10.1083/jcb.29.2.181. URL http://dx.doi.org/10.1083/jcb. 29 . 2.181 .

[212] Walter Liese. Tertiary wall and warty layer in wood cells. Journal of Polymer Science Part C: Polymer Symposia, 2(1):213-229, 1963. doi: https://doi.org/10.1002/polc. 5070020121. URL https://onlinelibrary.wiley.com/doi/abs/10.1002/polc. 5070020121.

[213] Mon-lin Kuo and Floyd G Manwiller. Morphological and chemical characteristics of the warty layer in red pine (pinus resinosa ait.). Wood and fiber science, 18(2): 239-247, 2007.

[214] N. Terashima, J. Nakashima, and K. Takabe. Proposed Structure for Protolignin in Plant Cell Walls, volume 697, pages 180-193. American Chemical Society, 2021/03/24 1998. ISBN 9780841235663. doi: doi:10.1021/bk-1998-0697.ch014. URL https://doi .org/10 .1021/bk-1998-0697. ch014.

[215] L. A. Donaldson. Mechanical constraints on lignin deposition during lignification. Wood Science and Technology, 28(2):111-118, 1994. doi: 10.1007/BF00192690. URL https://doi.org/10.1007/BF00192690. 


\section{Acknowledgements}

I would like to give my special thanks to many colleagues and friends at the Institute for Material Physics and the Institute of Wood Biology and Wood Products, who have helped me finish this thesis. I am very grateful to my supervisor Prof. Dr. Cynthia Volkert, for her scientific discussions, pronounced commitment, responsibility, guidance, compassion, and support in my work.

Special gratitude to Prof. Dr. Holger Militz, who helped me get introduced to numerous wood scientists in various conferences. Thanks for all of the enlightening conversation and his motivating comments during my Ph.D.

Many thanks to Dr. Mona Mass, my best friend, and colleague, who was always there for me during my Ph.D. with her friendship and kindness. Our collaboration was one of the most rewarding aspects of my doctoral study.

Dr. Maximilian Wentzel from the wood research group is gratefully acknowledged for his friendship, support, fruitful discussions, sample preparations, and sample treatments.

Special thanks to Dominik Tönnies, who taught me nanoindentation and supported me when I had problems and questions. He gave me excellent advice and encouragement when I was doing my Ph.D., which I really appreciate.

I would like to thank Dr. Hendrik Schmidt, who helped me with learning the AFM method. I want to thank Dr. Vladimirs Biziks for his assistance in preparing samples and learning about wood at the beginning of my Ph.D. I also thank Matthias Hahn, Tobias Schulz, Karin Ahlborn, and Kerstin Born for their support in all of the technical issues. I thank Christine Kuba so much for all her consideration and kindness, particularly for minimizing the 
difficulties of paperwork.

I want to thank my work community friends for creating an enjoyable working environment; more specifically, I would like to mention Torben Erichsen, Danny Schwarzbach, Niklas Weber, and Florian Schönewald. Thanks to my Iranian friends who helped me to feel to be at home in Germany.

I am most grateful to my husband, Mohsen, for his love, support, and compassion in my doctoral studies. I am thankful to him for showing me the joy of life and making my work more relaxed during the hard times of my thesis.

Last but not least, I am very grateful to my parents and my sisters for their help and support.

Salimeh Saleh 